\title{
Smooth affine group schemes over the dual numbers
}

\author{
Matthieu Romagny and Dajano Tossici
}

\begin{abstract}
In this article we provide an equivalence between the category of smooth affine group schemes over the ring of generalized dual numbers $k[I]$, and the category of extensions of the form $1 \rightarrow \operatorname{Lie}(G, I) \rightarrow E \rightarrow G \rightarrow 1$ where $G$ is a smooth affine group scheme over $k$. Here $k$ is an arbitrary commutative ring and $k[I]=k \oplus I$ with $I^{2}=0$. The equivalence is given by Weil restriction, and we provide a quasi-inverse which we call Weil extension. It is compatible with the exact structures and the $\mathbb{O}_{k}$-module stack structures on both categories. Our constructions rely on the use of the group algebra scheme of an affine group scheme; we introduce this object and establish its main properties. As an application, we establish a Dieudonné classification for smooth, commutative, unipotent group schemes over $k[I]$ when $k$ is a perfect field.
\end{abstract}

Keywords. Group scheme, deformation, dual numbers, adjoint representation, Weil restriction, Dieudonné classification of unipotent groups

2010 Mathematics Subject Classification. 14L15 (primary); 14D15, 14 G17 (secondary)

[Français]

Titre. Schémas en groupes affines et lisses sur les nombres duaux

Résumé. Nous construisons une équivalence entre la catégorie des schémas en groupes affines et lisses sur l'anneau des nombres duaux généralisés $k[I]$, et la catégorie des extensions de la forme $1 \rightarrow \operatorname{Lie}(G, I) \rightarrow E \rightarrow G \rightarrow 1$ où $G$ est un schéma en groupes affine, lisse sur $k$. Ici $k$ est un anneau commutatif arbitraire et $k[I]=k \oplus I$ avec $I^{2}=0$. L'équivalence est donnée par la restriction de Weil, et nous construisons un foncteur quasi-inverse explicite que nous appelons extension de Weil. Ces foncteurs sont compatibles avec les structures exactes et avec les structures de champs en $\mathbb{O}_{k}$-modules des deux catégories. Nos constructions s'appuient sur le schéma en algèbres de groupe d'un schéma en groupes affines, que nous introduisons et dont nous donnons les propriétés principales. En application, nous donnons une classification de Dieudonné pour les schémas en groupes commutatifs, lisses, unipotents sur $k[I]$ lorsque $k$ est un corps parfait.

Received by the Editors on August 30, 2018, and in final form on December 28, 2018. Accepted on March 4, 2019.

Matthieu Romagny

Université de Rennes, CNRS, IRMAR - UMR 6625, F-35000 Rennes, France

e-mail: matthieu.romagny@univ-rennes1.fr

Dajano Tossici

Université de Bordeaux, CNRS, Bordeaux INP, IMB, UMR 5251, F-33400, Talence, France

e-mail: Dajano.Tossici@math.u-bordeaux.fr 


\section{Contents}

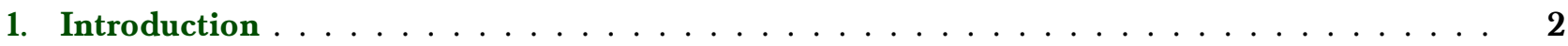

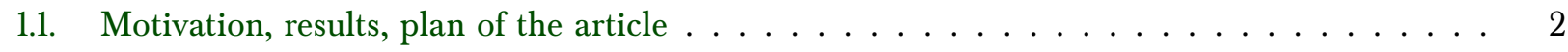

1.2. The $\mathbb{O}_{k}$-module stack structure of $\mathrm{Gr} / k[I]$ and $\operatorname{Ext}(I) / k \ldots \ldots \ldots \ldots$

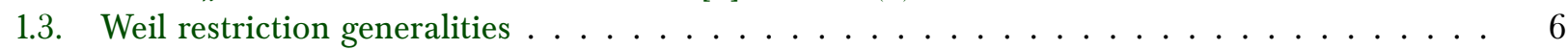

2. Group algebras of group schemes $\ldots \ldots \ldots \ldots \ldots \ldots \ldots$

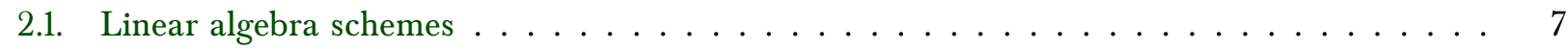

2.2. Group algebra: construction and examples $\ldots \ldots \ldots \ldots \ldots \ldots \ldots$

2.3. Properties: functoriality and adjointness $\ldots \ldots \ldots \ldots \ldots \ldots$

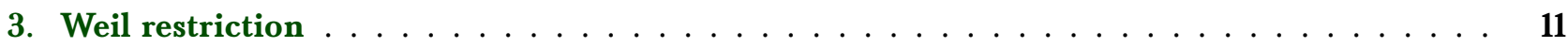

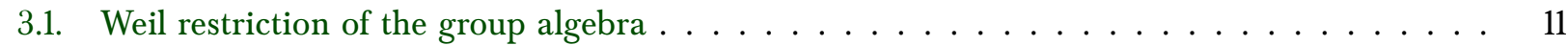

3.2. Kernel of the adjunction $\beta: h^{*} h_{*} \mathscr{G} \rightarrow \mathscr{G} \ldots \ldots \ldots \ldots \ldots$

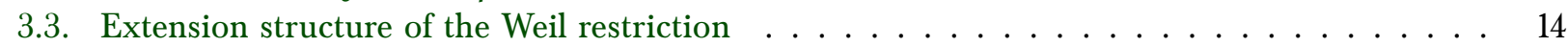

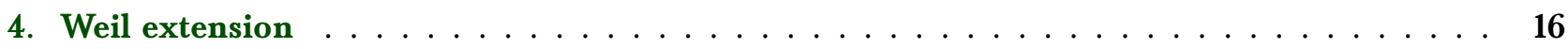

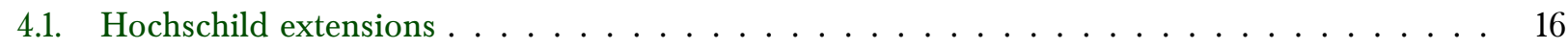

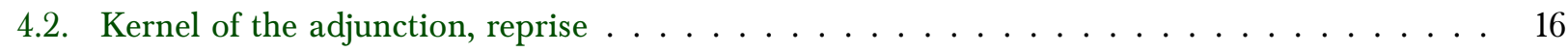

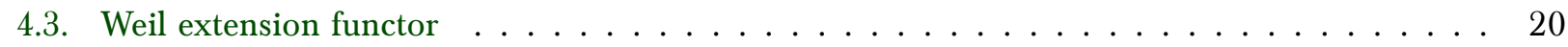

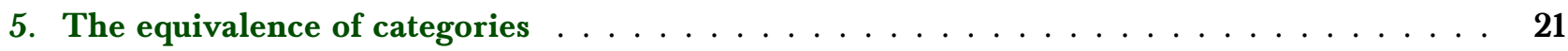

5.1. Equivariance of rigidifications under Lie algebra translation . . . . . . . . . . . . 21

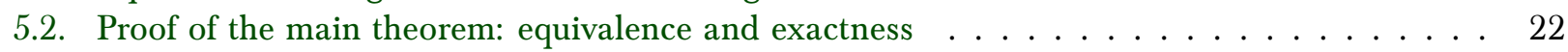

5.3. Proof of the main theorem: isomorphism of $\mathbb{O}_{k}$-module stacks $\ldots \ldots \ldots \ldots$. . . . . 24

6. Dieudonné theory for unipotent groups over the dual numbers $\ldots \ldots \ldots$

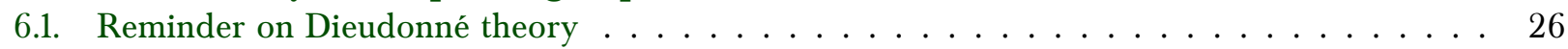

6.2. Dieudonné theory over the dual numbers $\ldots \ldots \ldots \ldots \ldots \ldots \ldots \ldots \ldots$

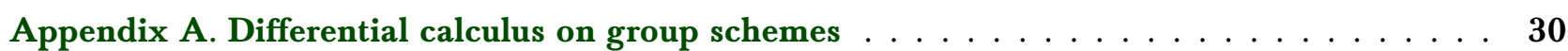

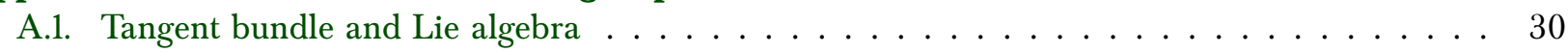

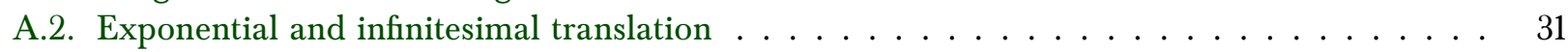

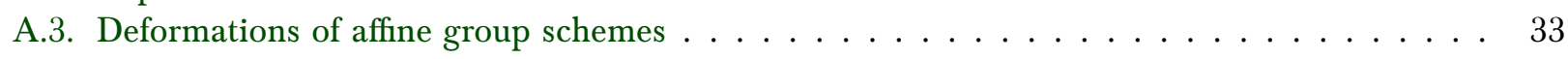

Appendix B. Module stacks in groupoids $\ldots \ldots \ldots \ldots \ldots \ldots$

\section{Introduction}

Throughout this article, we fix a commutative ring $k$ and a free $k$-module $I$ of finite rank $r \geqslant 1$. We consider the ring of (generalized) dual numbers $k[I]:=k \oplus I$ with $I^{2}=0$. We write $h: \operatorname{Spec}(k[I]) \rightarrow \operatorname{Spec}(k)$ the structure map and $i: \operatorname{Spec}(k) \rightarrow \operatorname{Spec}(k[I])$ the closed immersion. Also we denote by $\mathbb{O}_{k}$ the $\operatorname{Spec}(k)$-ring scheme such that if $R$ is a $k$-algebra then $\mathbb{O}_{k}(R)=R$ with its ring structure.

\subsection{Motivation, results, plan of the article}

1.1.1. Motivation. The starting point of our work is a relation between deformations and group extensions. To explain the idea, let $G$ be an affine, flat, finitely presented $k$-group scheme. It is shown in Illusie's book [1172] that the set of isomorphism classes of deformations of $G$ over $k[I]$ is in bijection with the cohomology group $\mathrm{H}^{2}\left(B G, \underline{\ell}_{G}^{\vee} \otimes I\right)$, see chap. VII, thm 3.2.1 in loc. cit. Here, the coefficients of cohomology are the derived dual of the equivariant cotangent complex $\underline{\ell}_{G} \in D(B G)$, tensored (in the derived sense) by $I$ 
viewed as the coherent sheaf it defines on the fpqc site of $B G$, also equal to the vector bundle $\mathbb{V}\left(I^{\vee}\right)$. If we assume moreover that $G$ is smooth, then the augmentation $\underline{\ell}_{G} \rightarrow \omega_{G}^{1}$ to the sheaf of invariant differential 1 -forms is a quasi-isomorphism and it follows that $\underline{\ell}_{G}^{\vee} \simeq$ Lie $G$. Since coherent cohomology of $B G$ is isomorphic to group cohomology of $G$, the cohomology group of interest ends up being $\mathrm{H}^{2}(G, \operatorname{Lie}(G, I))$ where $\operatorname{Lie}(G, I):=\operatorname{Lie} G \otimes \mathbb{V}\left(I^{\vee}\right)$. The latter cohomology group is meaningful also in the theory of group extensions, where it is known to classify isomorphism classes of extensions of $G$ by $\operatorname{Lie}(G, I)$ viewed as a $G$-module via the adjoint representation, see [DG70, chap. II, § 3, no 3.3 and III, § 6, no 2.1].

In this paper, our aim is to give a direct algebro-geometric construction of this correspondence between deformations and group extensions. Our main result is that the Weil restriction functor $h_{*}$ provides such a construction. Thereby, we obtain a categorification of a link that has been available only as a bijection between sets of isomorphism classes. This improvement is crucial for a better understanding of $k[I]$-group schemes, since in applications most groups occur as kernels or quotients of morphisms. We illustrate this by giving a Dieudonné-type theory for unipotent group schemes. Natural extensions of our result to more general thickenings of the base, or to non-smooth group schemes, would have further interesting applications. Since we wish to show our main results to the reader without further delay, we postpone the discussion of these applications to 1.1.4 below.

1.1.2. Results. Our main result is an equivalence between the category of smooth, affine $k[I]$-group schemes and a certain category of extensions of $k$-group schemes. However, for reasons that are discussed below, it is convenient for us to work with group schemes slightly more general than smooth ones. We say that a morphism of schemes $X \rightarrow S$ is differentially flat if both $X$ and $\Omega_{X / S}^{1}$ are flat over $S$. Examples of differentially flat group schemes include smooth group schemes, pullbacks from the spectrum of a field, Tate-Oort group schemes with parameter $a=0$ in characteristic $p$, flat vector bundles i.e. $\mathbb{V}(\mathscr{F})$ with $\mathscr{F}$ flat over the base, and truncated Barsotti-Tate groups of level $n$ over a base where $p^{n}=0$ [1185, 2.2.1]. If $\mathscr{G}$ is a $k[I]$-group scheme, we call rigidification of $\mathscr{G}$ an isomorphism of $k[I]$-schemes $\sigma: h^{*} \mathscr{G}_{k} \stackrel{\sim}{\rightarrow} \mathscr{G}$ that lifts the identity of the scheme $\mathscr{G}_{k}:=i^{*} \mathscr{G}$. Such a map need not be a morphism of group schemes. We say that $\mathscr{G}$ is rigid if it admits a rigidification. Examples of rigid group schemes include smooth group schemes, pullbacks from $\operatorname{Spec}(k)$, and groups of multiplicative type. Finally a deformation of a flat $k$-group scheme $G$ over $k[I]$ is a pair composed of a flat $k[I]$-group scheme $\mathscr{G}$ and an isomorphism of $k$-group schemes $\mathscr{G}_{k} \stackrel{\sim}{\rightarrow} G$.

Let $\mathrm{Gr} / k[I]$ be the category of affine, differentially flat, rigid $k[I]$-group schemes (this includes all smooth affine $k[I]$-group schemes). The morphisms in this category are the morphisms of $k[I]$-group schemes. Let $\operatorname{Ext}(I) / k$ be the category of extensions of the form $1 \rightarrow \operatorname{Lie}(G, I) \rightarrow E \rightarrow G \rightarrow 1$ where $G$ is an affine differentially flat $k$-group scheme, and "extension" means that $E \rightarrow G$ is an fpqc torsor under $\operatorname{Lie}(G, I)$. The morphisms in this category are the commutative diagrams:

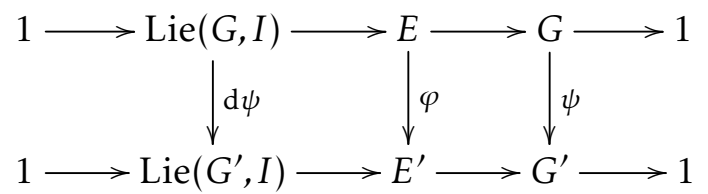

where $\mathrm{d} \psi=\operatorname{Lie}(\psi)$ is the differential of $\psi$. Usually such a morphism will be denoted simply $\varphi: E \rightarrow E^{\prime}$.

The categories $\mathrm{Gr} / k[I]$ and $\operatorname{Ext}(I) / k$ are exact categories. They are also fpqc stacks over $\operatorname{Spec}(k)$ equipped with the structure of $\mathbb{O}_{k}$-module stacks fibred in groupoids over $\mathrm{Gr} / k$. This means that there exist notions of sum and scalar multiple for objects of $\mathrm{Gr} / k[I]$ and $\operatorname{Ext}(I) / k$ (for extensions, the sum is the Baer sum); these structures are described in 1.2. We can now state our main result.

Theorem A (see 5.0.1). (1) The Weil restriction functor provides an equivalence:

$$
h_{*}: \mathrm{Gr} / k[I] \stackrel{\sim}{\longrightarrow} \operatorname{Ext}(I) / k .
$$

This equivalence commutes with base changes on $\operatorname{Spec}(k)$. 
(2) If $1 \rightarrow \mathscr{G}^{\prime} \rightarrow \mathscr{G} \rightarrow \mathscr{G}^{\prime \prime} \rightarrow 1$ is an exact sequence in $\mathrm{Gr} / k[I]$, then $1 \rightarrow h_{*} \mathscr{G}^{\prime} \rightarrow h_{*} \mathscr{G} \rightarrow h_{*} \mathscr{G}^{\prime \prime}$ is exact in $\operatorname{Ext}(I) / k$. If moreover $\mathscr{G}^{\prime}$ is smooth then $1 \rightarrow h_{*} \mathscr{G}^{\prime} \rightarrow h_{*} \mathscr{G} \rightarrow h_{*} \mathscr{G}^{\prime \prime} \rightarrow 1$ is exact. In particular, $h_{*}$ is an exact equivalence between the subcategories of smooth objects endowed with their natural exact structure.

(3) The equivalence $h_{*}$ is a morphism of $\mathbb{O}_{k}$-module stacks fibred over $\mathrm{Gr} / k$, i.e. it transforms the addition and scalar multiplication of deformations of a fixed $G \in \mathrm{Gr} / k$ into the Baer sum and scalar multiplication of extensions.

(4) Let $P$ be one of the properties of group schemes over a field: of finite type, smooth, connected, unipotent, split unipotent, solvable, commutative. Say that a group scheme over an arbitrary ring has property $P$ if it is flat and its fibres have $P$. Then $\mathscr{G} \in \mathrm{Gr} / k[I]$ has property $P$ if and only if the $k$-group scheme $E=h_{*} \mathscr{G}$ has $P$.

In order to show that $h_{*}$ is an equivalence, we build a quasi-inverse $h^{+}$which we call Weil extension. The construction and study of this functor is the hardest part of the proof.

As an application of the Theorem, we prove a Dieudonné classification for smooth, commutative, unipotent group schemes over the generalized dual numbers of a perfect field $k$. This takes the form of an exact equivalence of categories with a category of extensions of smooth, erasable Dieudonné modules. Here is the precise statement (we refer to Section 6 for the definition of all undefined terms).

Theorem B (see 6.2.6). Let SCU/k[I] be the category of smooth, commutative, unipotent $k[I]$-group schemes. Let $\mathbb{D}-I-M o d$ be the category of I-extensions of smooth erasable Dieudonné modules. Then the Dieudonné functor $\underline{M}: \mathrm{CU} / k \longrightarrow \mathbb{D}$-Mod induces a contravariant equivalence of categories:

$$
\mathscr{M}: \mathrm{SCU} / k[I] \longrightarrow \mathbb{D}-I-\operatorname{Mod}
$$

that sends $\mathscr{U}$ to the extension $\left.0 \rightarrow \underline{M}\left(\mathscr{U}_{k}\right) \rightarrow \underline{M}\left(h_{*} \mathscr{U}\right)\right) \rightarrow \underline{M}\left(\operatorname{Lie}\left(\mathscr{U}_{k}, I\right)\right) \rightarrow 0$.

1.1.3. Comments. An important tool in many of our arguments is the group algebra scheme. It provides a common framework to conduct computations in the groups and their tangent bundles simultaneously. It allows us to describe conveniently the Weil restriction of a group scheme, and is essential in the proof of Theorem A. Since we are not aware of any treatment of the group algebra scheme in the literature, we give a detailed treatment in Section 2. We point out that this concept is useful in other situations; in particular it allows to work out easily the deformation theory of smooth affine group schemes, as we show in Subsection A.3.

Let us say a word on the assumptions. The choice to work with differentially flat group schemes instead of simply smooth ones is not just motivated by the search for maximal generality or aesthetic reasons. It is also extremely useful because when working with an affine, smooth group scheme $G$, we use our results also for the group algebra $\mathbb{O}_{k}[G]$ in the course of proving the main theorem; and the group algebra $\mathbb{O}_{k}[G]$ is differentially flat and rigid, but usually infinite-dimensional and hence not smooth.

There are at least two advantages to work over generalized dual numbers $k[I]$ rather than simply the usual ring $k[\varepsilon]$ with $\varepsilon^{2}=0$. The first is that in order to prove that our equivalence of categories respects the $\mathbb{O}_{k}$-module stack structure, we have to introduce the ring $k[I]$ with the two-dimensional $k$-module $I=k \varepsilon+k \varepsilon^{\prime}$. Indeed, this is needed to describe the sum of deformations and the Baer sum of extensions. The other advantage is that since arbitrary local Artin $k$-algebras are filtered by Artin $k$-algebras whose maximal ideal has square zero, our results may be useful in handling deformations along more general thickenings.

1.1.4. Further developments. Our results have several desirable generalizations. Here are the two most natural directions. First, one may wish to relax the assumptions on the group schemes and consider nonaffine or non-smooth group schemes; second one may wish to consider more general thickenings than that given by the dual numbers. Let us explain how our personal work indicates a specific axis for research. In previous work of the authors with Ariane Mézard [MRT13], we studied models of the group schemes of roots of unity $\mu_{p^{n}}$ over $p$-adic rings. As a result, we raised a conjecture which says in essence that every 
such model can be equipped with a cohomological theory that generalizes the Kummer theory available on the generic fibre. In the process of trying to prove the conjecture, we encountered various character groups of smooth and finite unipotent group schemes over truncated discrete valuation rings. In order to compute these, it is therefore desirable to obtain a statement similar to Theorem A in this context. The present paper can be seen as the first part of this programme, carried out in the simplest case; we plan to realize the second part of the programme by using the derived Weil restriction or derived Greenberg functor in place of the usual Weil restriction.

1.1.5. Plan of the article. The present Section 1 ends with material of preliminary nature on the description of the $\mathbb{O}_{k}$-module stack structure of the categories $\mathrm{Gr} / k[I]$ and $\operatorname{Ext}(I) / k$ and on Weil restriction. In Section 2 we introduce group algebra schemes. In Section 3 we describe the functor $h_{*}: \mathrm{Gr} / k[I] \rightarrow \operatorname{Ext}(I) / k$, in Section 4 we construct a functor $h^{+}: \operatorname{Ext}(I) / k \rightarrow \mathrm{Gr} / k[I]$, while in Section 5 we prove that these functors are quasi-inverse and we complete the proof of Theorem A. Finally, in Section 6 we derive the Dieudonné classification for smooth commutative unipotent group schemes over the dual numbers. In the Appendices, we review notions from differential calculus (tangent bundle, Lie algebra and exponentials) and module categories (Picard categories with scalar multiplication) in the level of generality needed in the paper.

1.1.6. Acknowledgements. For various discussions and remarks, we thank Sylvain Brochard, Xavier Caruso, Brian Conrad, Bernard Le Stum, Brian Osserman, and Tobias Schmidt. We acknowledge the help of the referee to make the article much more incisive. We are also grateful to the CIRM in Luminy where part of this research was done. Finally, the first author would like to thank the executive and administrative staff of IRMAR and of the Centre Henri Lebesgue ANR-11-LABX-0020-01 for creating an attractive mathematical environment.

\subsection{The $\mathbb{O}_{k}$-module stack structure of $\mathrm{Gr} / k[I]$ and $\operatorname{Ext}(I) / k$}

Both categories $\mathrm{Gr} / k[I]$ and $\operatorname{Ext}(I) / k$ are endowed with the structure of $\mathbb{O}_{k}$-module stacks in groupoids over $\mathrm{Gr} / k$. The reader who wishes to see the full-fledged definition is invited to read Appendix B. In rough terms, once a $k$-group scheme $G$ is fixed, the $\mathbb{O}_{k}$-module category structure boils down to an addition law by which one can add deformations (resp. extensions) of $G$, and an external law by which one can multiply a deformation (resp. an extension) by scalars of the ring scheme $\mathbb{O}_{k}$. Here is a description of these structures.

1.2.1. The $\mathbb{O}_{k}$-module stack $\mathrm{Gr} / k[I] \rightarrow \mathrm{Gr} / k$. Let $G \in \mathrm{Gr} / k$ be fixed. Let $\mathscr{G}_{1}, \mathscr{G}_{2} \in \mathrm{Gr} / k[I]$ with identifications $i^{*} \mathscr{G}_{1} \simeq G \simeq i^{*} \mathscr{G}_{2}$. The addition is obtained by a two-step process. First we glue these group schemes along their common closed subscheme $G$ :

$$
\mathscr{G}^{\prime}:=\mathscr{G}_{1} \amalg_{G} \mathscr{G}_{2}
$$

This lives over the scheme $\operatorname{Spec}(k[I]) \times_{\operatorname{Spec}(k)} \operatorname{Spec}(k[I])=\operatorname{Spec}(k[I \oplus I])$. The properties of gluing along infinitesimal thickenings are studied in the Stacks Project [SP]. We point out some statements relevant to our situation: existence of the coproduct in Tag 07RV, a list of properties preserved by gluing in Tag 07RX, gluing of modules in Tag 08KU and preservation of flatness of modules in Tag 07RW. It follows from these results that $\mathscr{G}^{\prime}$ is an object of $\mathrm{Gr} / k[I \oplus I]$. Then we form the desired sum

$$
\mathscr{G}_{1}+\mathscr{G}_{2}:=j^{*}\left(\mathscr{G}^{\prime}\right)=j^{*}\left(\mathscr{G}_{1} \amalg_{G} \mathscr{G}_{2}\right)
$$

by pullback along the closed immersion

$$
j: \operatorname{Spec}(k[I]) \longleftrightarrow \operatorname{Spec}(k[I \oplus I])
$$

induced by the addition morphism $I \oplus I \rightarrow I, i_{1} \oplus i_{2} \mapsto i_{1}+i_{2}$. The neutral element for this addition is the group scheme $h^{*} G$. The scalar multiple

$$
\lambda \mathscr{G}:=s_{\lambda}^{*} \mathscr{G}
$$


is given by rescaling using the scheme map $s_{\lambda}: \operatorname{Spec}(k[I]) \rightarrow \operatorname{Spec}(k[I])$ induced by the $k$-algebra map $k[I] \rightarrow k[I]$ which is multiplication by $\lambda$ in $I$. The axioms of $\mathbb{O}_{k}$-module stacks can be checked but we leave the details to the courageous reader.

1.2.2. The $\mathbb{O}_{k}$-module stack $\operatorname{Ext}(I) / k \rightarrow \mathrm{Gr} / k$. Again let $G \in \mathrm{Gr} / k$ be fixed and set $L:=\operatorname{Lie}(G, I)$. Let $E_{1}, E_{2} \in \mathcal{E} x t(G, L)$ be two extensions. Their addition is given by Baer sum; here again this is a two-step process. Namely, we first form the fibre product $E^{\prime}=E_{1} \times_{G} E_{2}$ which is an extension of $G$ by $L \times L$. Then the Baer sum is the pushforward of this extension along the addition map $+: L \times L \rightarrow L$. All in all, we have the following diagram which serves as a definition of $E_{1}+E_{2}$ :

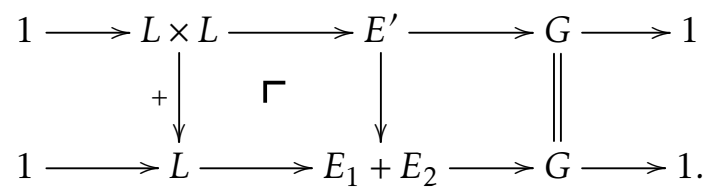

Explicitly, the underlying group scheme of the Baer sum is given by $E_{1}+E_{2}=E^{\prime} / M$ where

$$
M=\operatorname{ker}(L \times L \rightarrow L)=\{(x,-x), x \in L\} .
$$

The neutral element for this addition is the trivial extension $E=L \rtimes G$. Even though this is not emphasized in the literature, the usual proofs of the fact that the set of extensions endowed with the Baer sum operation is an abelian group provide explicit associativity and commutativity constraints proving that $\mathcal{E} x t(G, L)$ is a Picard category. The associativity constraint is obtained by expressing $\left(E_{1}+E_{2}\right)+E_{3}$ and $E_{1}+\left(E_{2}+E_{3}\right)$ as isomorphic quotients of $E_{1} \times{ }_{G} E_{2} \times_{G} E_{3}$, and the commutativity constraint is obtained from the flipping morphism in $E_{1} \times_{G} E_{2}$. The scalar multiplication by $\lambda \in k$ is given by pushforward along the multiplicationby- $\lambda$ morphism in the module scheme $L=\operatorname{Lie}(G, I)$. All in all, we have the following diagram which serves as a definition of $\lambda E$ :

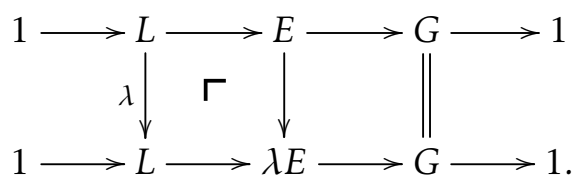

Again, the verification of the axioms of an $\mathbb{O}_{k}$-module stack is tedious but not difficult.

\subsection{Weil restriction generalities}

We briefly give the main definitions and notations related to Weil restriction; we refer to [BLR90, § 7.6] for more details. Let $h: \operatorname{Spec}\left(k^{\prime}\right) \rightarrow \operatorname{Spec}(k)$ be a finite, locally free morphism of affine schemes. Let $(\mathrm{Sch} / k)$ be the category of $k$-schemes and (Fun $/ k$ ) the category of functors $(\mathrm{Sch} / k)^{\circ} \rightarrow(\mathrm{Sets})$. The Yoneda functor embeds the former category into the latter. By sending a morphism of functors $f: X^{\prime} \rightarrow \operatorname{Spec}\left(k^{\prime}\right)$ to the morphism $h \circ f: X^{\prime} \rightarrow \operatorname{Spec}(k)$ we obtain a functor $h_{!}:\left(\right.$Fun $\left./ k^{\prime}\right) \rightarrow($ Fun $/ k)$. Sometimes we will refer to $h_{!} X^{\prime}$ as the $k^{\prime}$-functor $X^{\prime}$ viewed as a $k$-functor and the notation $h_{!}$will be omitted. The pullback functor $h^{*}:($ Fun $/ k) \rightarrow\left(\right.$ Fun $\left./ k^{\prime}\right)$ is right adjoint to $h_{!}$, in particular $\left(h^{*} X\right)\left(S^{\prime}\right)=X\left(h_{!} S^{\prime}\right)$ for all $k^{\prime}$-schemes $S^{\prime}$. The Weil restriction functor $h_{*}:\left(\right.$ Fun $\left./ k^{\prime}\right) \rightarrow($ Fun $/ k)$ is right adjoint to $h^{*}$, in particular we have $\left(h_{*} X^{\prime}\right)(S)=X^{\prime}\left(h^{*} S\right)$ for all $k$-schemes $S$. Thus we have a sequence of adjoint functors:

$$
h_{!}, h^{*}, h_{*} \text {. }
$$

The functors $h_{!}$and $h^{*}$ preserve the subcategories of schemes. The same is true for $h_{*}$ if $h$ is radicial, a case which covers our needs (see [BLR90, § 7.6] for refined representability results). We write

$$
\alpha: 1 \longrightarrow h_{*} h^{*} \text { and } \beta: h^{*} h_{*} \longrightarrow 1
$$

for the unit and counit of the $\left(h^{*}, h_{*}\right)$-adjunction. If $X$ is a separated $k$-scheme then $\alpha_{X}: X \rightarrow h_{*} h^{*} X$ is a closed immersion. If $X^{\prime}$ is a $k^{\prime}$-group (resp. algebra) functor (resp. scheme), then also $h_{*} X^{\prime}$ is a $k$-group 
(resp. algebra) functor (resp. scheme). If moreover $X^{\prime} \rightarrow \operatorname{Spec}\left(k^{\prime}\right)$ is smooth of relative dimension $n$, then $h_{*} X^{\prime} \rightarrow \operatorname{Spec}(k)$ is smooth of relative dimension $n\left[k^{\prime}: k\right]$ where $\left[k^{\prime}: k\right]$ is the locally constant rank of $h$. Quite often, it is simpler to consider functors defined on the subcategory of affine schemes; the functors $h_{!}$, $h^{*}, h_{*}$ are defined similarly in this context.

\section{Group algebras of group schemes}

Let $G$ be an affine $k$-group scheme. In this subsection, we explain the construction of the group algebra $\mathbb{O}_{k}[G]$, which is the analogue in the setting of algebraic geometry of the usual group algebra of abstract discrete groups. Note that for a finite constant group scheme, the set $\mathbb{O}_{k}[G](k)$ of $k$-rational points of the group algebra and the usual group algebra $k[G]$ are isomorphic, but for other groups they do not have much in common in general; this will be emphasized below. Since we are not aware of any appearance of the group algebra in the literature, we give a somewhat detailed treatment, including examples (2.2.4-2.2.6), basic properties (2.3.1) and the universal property (2.3.2).

\subsection{Linear algebra schemes}

2.1.1. Vector bundles. Let $S=\operatorname{Spec}(k)$. As in [EGA $1_{\text {new }}$, we call vector bundle an $\mathbb{O}_{k}$-module scheme of the form $\mathbb{V}(\mathscr{F})=\operatorname{Spec} S(\mathscr{F})$ where $\mathscr{F}$ is a quasi-coherent $\mathcal{O}_{S}$-module and $\mathrm{S}(\mathscr{F})$ is its symmetric algebra. We say smooth vector bundle if $\mathscr{F}$ is locally free of finite rank. If $f: X \rightarrow S$ is quasi-compact and quasiseparated, we set $\mathbb{V}(X / S):=\mathbb{V}\left(f_{*} \mathcal{O}_{X}\right)$ so $\mathbb{V}(X / S)(T)=\operatorname{Hom}_{\mathcal{O}_{T}-\operatorname{Mod}}\left(\left(f_{*} \mathcal{O}_{X}\right)_{T}, \mathcal{O}_{T}\right)$ for all $S$-schemes $T$. There is a canonical $S$-morphism $v_{X}: X \longrightarrow \mathbb{V}(X / S)$ which is initial among all $S$-morphisms from $X$ to a vector bundle. We call it the vector bundle envelope of $X / S$. If $X$ is affine over $S$, the map $v_{X}$ is a closed immersion because it is induced by the surjective morphism of algebras $S\left(f_{*} \mathcal{O}_{X}\right) \longrightarrow f_{*} \mathcal{O}_{X}$ induced by the identity $f_{*} \mathcal{O}_{X} \longrightarrow f_{*} \mathcal{O}_{X}$.

2.1.2. Base change and ring scheme. If $h: \operatorname{Spec}\left(k^{\prime}\right) \rightarrow \operatorname{Spec}(k)$ is a morphism of affine schemes, there is a morphism of $k^{\prime}$-ring schemes $h^{*} \mathbb{O}_{k} \rightarrow \mathbb{O}_{k^{\prime}}$ which is the identity on points, hence an isomorphism of ring schemes. However, whereas the target has a natural structure of $\mathbb{O}_{k^{\prime}}$-algebra, the source does not. For this reason, the pullback of module functors or module schemes along $h$ is defined as $M \mapsto h^{*} M \otimes_{h^{*}} \mathbb{O}_{k} \mathbb{O}_{k^{\prime}}$, as is familiar for the pullback of modules on ringed spaces. Usually we will write simply $h^{*} M$.

For later use, we give some complements on the case where $k^{\prime}=k[I]$ is the ring of generalized dual numbers, for some finite free $k$-module $I$. We will identify $I$ and its dual $I^{\vee}=\operatorname{Hom}_{k}(I, k)$ with the coherent $\mathcal{O}_{\text {Spec }(k)}$-modules they define, thus we have the vector bundle $\mathbb{V}\left(I^{\vee}\right)$. For each $k$-algebra $R$, we have $R[I]=R \oplus I \otimes_{k} R$ where $I R \simeq I \otimes_{k} R$ has square 0 . This decomposition functorial in $R$ gives rise to a direct sum decomposition of $\mathbb{O}_{k}$-module schemes:

$$
h_{*} \mathbb{O}_{k[I]}=\mathbb{O}_{k} \oplus \mathbb{V}\left(I^{\vee}\right) .
$$

It is natural to use the notation $\mathbb{O}_{k}\left[\mathbb{V}\left(I^{\vee}\right)\right]$ for the $\mathbb{O}_{k}$-algebra scheme on the right-hand side, however we will write more simply $\mathbb{O}_{k}[I]$. Now we move up on $\operatorname{Spec}(k[I])$, where there is a morphism of $\mathbb{O}_{k[I]}$-module schemes $h^{*} \mathbb{V}\left(I^{\vee}\right) \rightarrow \mathbb{O}_{k[I]}$ defined for all $k[I]$-algebras $R$ as the morphism $I \otimes_{k} R \rightarrow R, i \otimes x \mapsto i_{R} x$ where $i_{R}:=i 1_{R}$. According to what we said before, the pullback $h^{*} \mathbb{V}\left(I^{\vee}\right)$ has the module structure such that a section $a \in \mathbb{O}_{k[I]}$ acts by $a \cdot i \otimes x:=i \otimes a x$. The image of $h^{*} \mathbb{V}\left(I^{\vee}\right) \rightarrow \mathbb{O}_{k[I]}$ is the ideal $I \cdot \mathbb{O}_{k[I]}$ defined by $\left(I \cdot \mathbb{O}_{k[I]}\right)(R)=I R$ for all $k[I]$-algebras $R$. There is an exact sequence of $\mathbb{O}_{k[I]}$-module schemes:

$$
0 \longrightarrow I \cdot \mathbb{O}_{k[I]} \longrightarrow \mathbb{O}_{k[I]} \longrightarrow i_{*} \mathbb{O}_{k} \longrightarrow 0 .
$$


2.1.3. $\mathbb{O}_{k}$-Algebra schemes. Here we define a category $\left(\mathbb{O}_{k}\right.$ - $\left.\mathrm{Alg}\right)$ of linear $\mathbb{O}_{k}$-algebra schemes and give a summary of elementary properties. For us an $\mathbb{O}_{k}$-algebra scheme is a $k$-scheme $D$ endowed with two internal composition laws,$+ \times: D \times D \rightarrow D$ called addition and multiplication possessing two neutral sections $0,1: \operatorname{Spec}(k) \rightarrow D$, and an external law $\cdot: \mathbb{O}_{k} \times D \rightarrow D$, such that for each $k$-algebra $R$ the tuple $(D(R),+, \times, 0,1, \cdot)$ is an associative unitary $R$-algebra. In particular $(D,+, 0)$ is a commutative group scheme, and $(D(R), \times, 1)$ is a (possibly noncommutative) monoid. We say that $D$ is a linear $\mathbb{O}_{k}$-algebra scheme if its underlying $\mathbb{O}_{k}$-module scheme is a vector bundle. In this case $\mathscr{F}$ can be recovered as the "dual bundle" sheaf $\mathscr{F}=\operatorname{Hom}_{\mathbb{O}_{k} \text {-Mod }}\left(D, \mathbb{O}_{k}\right)$, the Zariski sheaf over $S$ whose sections over an open $U$ are the morphisms of $\mathbb{O}_{k \mid U}$-modules $D_{\mid U} \rightarrow \mathbb{O}_{k \mid U}$ (read [EGA1 $1_{\text {new }}, 9.4 .9$ ] between the lines). For an affine $\mathbb{O}_{k}$-algebra scheme in our sense, the comultiplication is a map $\Delta: S(\mathscr{F}) \rightarrow S(\mathscr{F}) \otimes S(\mathscr{F})$ and the bilinearity of $m$ implies that this map is induced from a map $\Delta_{0}: \mathscr{F} \rightarrow \mathscr{F} \otimes \mathscr{F}$. Finally we point out two constructions on linear $\mathbb{O}_{k}$-algebra schemes. The first is the tensor product $D \otimes_{\mathbb{O}_{k}} D^{\prime}$, which as a functor is defined as $R \mapsto D(R) \otimes_{R} D^{\prime}(R)$. If $D=\mathbb{V}(\mathscr{F})$ and $\left.D^{\prime}=\mathbb{V}(\mathscr{F})^{\prime}\right)$ with $\mathscr{F}, \mathscr{F}^{\prime}$ locally free of finite ranks, then $D \otimes_{\mathbb{O}_{k}} D^{\prime} \simeq \mathbb{V}\left(\mathscr{F} \otimes \mathscr{F}^{\prime}\right)$. The second construction is the group of units. We observe that for any linear $\mathbb{O}_{k}$-algebra scheme $D$, the subfunctor $D^{\times} \subset D$ of (multiplicative) units is the preimage under the multiplication $D \times D \rightarrow D$ of the unit section $1: \operatorname{Spec}(k) \rightarrow D$ and is therefore representable by an affine scheme. This gives rise to the group of units functor $\left(\mathbb{O}_{k}\right.$ - Alg $) \rightarrow(k-\mathrm{Gr}), D \mapsto D^{\times}$where $(k-\mathrm{Gr})$ is the category of affine $k$-group schemes.

2.1.4. Remark. We do not know if an $\mathbb{O}_{k}$-algebra scheme whose underlying scheme is affine over $S$ is always of the form $\mathbb{V}(\mathscr{F})$.

\subsection{Group algebra: construction and examples}

Let $G=\operatorname{Spec}(A)$ be an affine $k$-group scheme. We write $(u, v) \mapsto u \star v$ or sometimes simply $(u, v) \mapsto u v$ the multiplication of $G$. This operation extends to the vector bundle envelope $\mathbb{V}(G / k)$, as follows. Let $\Delta: A \rightarrow A \otimes_{k} A$ be the comultiplication. For each $k$-algebra $R$, we have $\mathbb{V}(G / k)(R)=\operatorname{Hom}_{k-\operatorname{Mod}}(A, R)$. If $u, v: A \rightarrow R$ are morphisms of $k$-modules, we consider the composition $u \star v:=(u \otimes v) \circ \Delta$ :

$$
u \star v: A \stackrel{\Delta}{\longrightarrow} A \otimes_{k} A \stackrel{u \otimes v}{\longrightarrow} R .
$$

Here the map $u \otimes v: A \otimes_{k} A \rightarrow R$ is $a \otimes b \mapsto u(a) v(b)$.

2.2.1. Definition. The group algebra of the k-group scheme $G$ :

$$
\mathbb{O}_{k}[G]:=(\mathbb{V}(G / k),+, \star),
$$

is the vector bundle $\mathbb{V}(G / k)$ endowed with the product just defined. We write $v_{G}: G \hookrightarrow \mathbb{O}_{k}[G]$ for the closed immersion as in paragraph 2.1.1.

We check below that $\mathbb{O}_{k}[G]$ is a linear $\mathbb{O}_{k}$-algebra scheme. Apart from $G(R)$, there is another noteworthy subset inside $\mathbb{O}_{k}[G](R)$, namely the set $\operatorname{Der}_{G}(R)$ of $k$-module maps $d: A \rightarrow R$ which are $u$-derivations for some $k$-algebra map $u: A \rightarrow R$ (which need not be determined by $d$ ); a more accurate notation would be $\operatorname{Der}\left(\mathcal{O}_{G}, \mathbb{O}_{k}\right)(R)$ but we favour lightness. Here are the first basic properties coming out of the construction.

2.2.2. Proposition. Let $G$ be an affine $k$-group scheme. Let $\mathbb{O}_{k}[G]$ and $\operatorname{Der}_{G}$ be as described above.

(1) The tuple $\mathbb{O}_{k}[G]:=(\mathbb{V}(G / k),+, \star)$ is a linear $\mathbb{O}_{k}$-algebra scheme.

(2) As a $k$-scheme, $\mathbb{O}_{k}[G]$ is $k$-flat (resp. has $k$-projective function ring) iff $G$ has the same property.

(3) The composition $G \hookrightarrow \mathbb{O}_{k}[G]^{\times} \hookrightarrow \mathbb{O}_{k}[G]$ is a closed embedding, hence also $G \hookrightarrow \mathbb{O}_{k}[G]^{\times}$.

(4) The subfunctor $\operatorname{Der}_{G} \subset \mathbb{O}_{k}[G]$ is stable by multiplication by $G$ on the left and on the right, so it acquires left and right $G$-actions. More precisely, if $u, v: A \rightarrow R$ are maps of algebras, $d: A \rightarrow R$ is a $u$-derivation and $d^{\prime}: A \rightarrow R$ is a $v$-derivation, then $d \star v$ and $u \star d^{\prime}$ are $(u \star v)$-derivations.

Proof. Omitted. 
2.2.3. Remark on Hopf algebra structure. If $G$ is a finite, locally free commutative $k$-group scheme, then the multiplication of its function ring induces a comultiplication on $\mathbb{O}_{k}[G]$ making it into a Hopf $\mathbb{O}_{k}$-algebra scheme. Moreover the $k$-algebra $\mathbb{O}_{k}[G](k)$ is the ring of functions of the Cartier dual $G^{\vee}$. This Hopf algebra structure highlights Examples 2.2.5 and 2.2.6 below.

2.2.4. Example 1: finite locally free groups. If $G$ is a finite locally free $k$-group scheme, the algebra $\mathbb{O}_{k}[G]$ is a smooth vector bundle of $\operatorname{rank}[G: \operatorname{Spec}(k)]$ and its group of units is the complement in $\mathbb{O}_{k}[G]$ of the Cartier divisor equal to the zero locus of the determinant of the left regular representation:

$$
\mathbb{O}_{k}[G] \stackrel{\mathbb{L}}{\longrightarrow} \operatorname{End}_{\mathbb{O}_{k}-\operatorname{Mod}}\left(\mathbb{O}_{k}[G]\right) \stackrel{\text { det }}{\longrightarrow} \mathbb{A}^{1} .
$$

If moreover $G$ is the finite constant $k$-group scheme defined by a finite abstract group $\Gamma$, then $\mathbb{O}_{k}[G]$ is isomorphic to the algebra scheme defined by the abstract group algebra $k[\Gamma]$, that is to say $\mathbb{O}_{k}[G](R) \simeq R[\Gamma]$ functorially in $R$.

2.2.5. Example 2: the additive group. Let $G=\mathbb{G}_{a}=\operatorname{Spec}(k[x])$. For a $k$-algebra $R$, let

$$
R\langle\langle t\rangle\rangle=R\left[\left[t, t^{[2]}, t^{[3]}, \ldots\right]\right]
$$

be the $R$-algebra of divided power formal power series in one variable $t$, with $t^{[i]} t^{[j]}=\left(\begin{array}{c}i+j \\ i\end{array}\right) t^{[i+j]}$ for all $i, j \geqslant 0$. Setting $\mathbb{O}\langle\langle t\rangle\rangle(R)=R\langle\langle t\rangle\rangle$, we have an isomorphism $\mathbb{O}_{k}[G] \stackrel{\sim}{\rightarrow} \mathbb{O}\langle\langle t\rangle\rangle$ given by:

$$
\mathbb{O}_{k}[G](R) \stackrel{\sim}{\rightarrow} R\langle\langle t\rangle\rangle \quad, \quad(k[x] \stackrel{u}{\longrightarrow} R) \longmapsto \sum_{i \geqslant 0} u\left(x^{i}\right) t^{i} .
$$

If $k$ is a ring of characteristic $p>0$, let $H=\alpha_{p}$ be the kernel of Frobenius. The algebra $\mathbb{O}_{k}[H](R)$ is identified with the $R$-subalgebra of $R\langle\langle t\rangle\rangle$ generated by $t$, which is isomorphic to $R[t] /\left(t^{p}\right)$ because $t^{p}=p t^{[p]}=0$.

2.2.6. Example 3: the multiplicative group. Let $G=\mathbb{G}_{m}=\operatorname{Spec}(k[x, 1 / x])$. Let $R^{\mathbb{Z}}$ be the product algebra, whose elements are sequences with componentwise addition and multiplication. We have an isomorphism $\mathbb{O}_{k}[G] \stackrel{\sim}{\rightarrow} \prod_{i \in \mathbb{Z}} \mathbb{O}_{k}$ given by:

$$
\mathbb{O}_{k}[G](R) \stackrel{\sim}{\longrightarrow} R^{\mathbb{Z}} \quad, \quad(k[x] \stackrel{u}{\longrightarrow} R) \longmapsto\left\{u\left(x^{i}\right)\right\}_{i \in \mathbb{Z}} .
$$

More generally, for any torus $T$ with character group $X(T)$, we have $\mathbb{O}_{k}[T] \stackrel{\sim}{\rightarrow} \prod_{i \in X(T)} \mathbb{O}_{k}$. Let $H=\mu_{n}$ be the subgroup of $n$-th roots of unity. The algebra $\mathbb{O}_{k}[H](R)$ is identified with the $R$-subalgebra of $\mathbb{Z} / n \mathbb{Z}$-invariants $\mathbb{O}_{k}[G](R)^{\mathbb{Z} / n \mathbb{Z}}$, composed of sequences $\left\{r_{i}\right\}_{i \in \mathbb{Z}}$ such that $r_{i+n j}=r_{i}$ for all $i, j \in \mathbb{Z}$.

\subsection{Properties: functoriality and adjointness}

Here are some functoriality properties of the group algebra.

2.3.1. Proposition. Let $G$ be an affine $k$-group scheme. The formation of the group algebra $\mathbb{O}_{k}[G]$ :

(1) is functorial in $G$ and faithful;

(2) commutes with base change $k^{\prime} / k$;

(3) is compatible with products: there is a canonical isomorphism $\mathbb{O}_{k}[G] \otimes_{\mathbb{O}_{k}} \mathbb{O}_{k}[H] \stackrel{\sim}{\rightarrow} \mathbb{O}_{k}[G \times H]$;

(4) is compatible with Weil restriction: if $h: \operatorname{Spec}\left(k^{\prime}\right) \rightarrow \operatorname{Spec}(k)$ is a finite locally free morphism of schemes, there is an isomorphism of $\mathbb{O}_{k}$-algebra schemes $\mathbb{O}_{k}[G] \otimes_{\mathbb{O}_{k}} h_{*} \mathbb{O}_{k^{\prime}} \stackrel{\sim}{\rightarrow} h_{*} h^{*} \mathbb{O}_{k}[G]$. 
Proof. (1) Any map of affine $k$-group schemes $G=\operatorname{Spec}(A) \rightarrow H=\operatorname{Spec}(B)$ gives rise to a map of $k$-Hopf algebras $B \rightarrow A$ and then by precomposition to a map of $R$-algebras $\mathbb{O}_{k}[G](R) \rightarrow \mathbb{O}_{k}[H](R)$ which is functorial in $R$. Faithfulness follows from the fact that $G \hookrightarrow \mathbb{O}_{k}[G]$ is a closed immersion.

(2) The isomorphism $\operatorname{Hom}_{k^{\prime}-\operatorname{Mod}}\left(A \otimes_{k} k^{\prime}, R^{\prime}\right) \stackrel{\sim}{\rightarrow} \operatorname{Hom}_{k \text {-Mod }}\left(A, R^{\prime}\right)$, functorial in the $k^{\prime}$-algebra $R^{\prime}$, gives an isomorphism $\mathbb{O}_{k^{\prime}}\left[G_{k^{\prime}} \stackrel{\sim}{\rightarrow} \mathbb{O}_{k}[G] \times \operatorname{Spec}(k) \operatorname{Spec}\left(k^{\prime}\right)\right.$.

(3) Write $G=\operatorname{Spec}(A)$ and $H=\operatorname{Spec}(B)$. To a pair of $k$-module maps $u: A \rightarrow R$ and $v: B \rightarrow R$ we attach the map $u \otimes v: A \otimes_{k} B \rightarrow R, a \otimes b \mapsto u(a) v(b)$. This defines an isomorphism

$$
\operatorname{Hom}_{k-\operatorname{Mod}}(A, R) \otimes_{R} \operatorname{Hom}_{k-\operatorname{Mod}}(B, R) \stackrel{\sim}{\rightarrow} \operatorname{Hom}_{k-\operatorname{Mod}}\left(A \otimes_{k} B, R\right)
$$

which is functorial in $R$. The result follows.

(4) If $D$ is an $\mathbb{O}_{k}$-algebra scheme, the functorial $R$-algebra maps

$$
\begin{aligned}
D(R) \otimes_{R}\left(R \otimes_{k} k^{\prime}\right) & \longrightarrow D\left(R \otimes_{k} k^{\prime}\right) \\
d \otimes r^{\prime} & \longmapsto r^{\prime} d
\end{aligned}
$$

fit together to give a morphism $D \otimes_{\mathbb{O}_{k}} h_{*} \mathbb{O}_{k^{\prime}} \rightarrow h_{*} h^{*} D$. In case $D=\mathbb{O}_{k}[G]$ and $h$ finite locally free, this is none other than the isomorphism $\operatorname{Hom}_{k-\operatorname{Mod}}(A, R) \otimes_{k} k^{\prime} \stackrel{\sim}{\rightarrow} \operatorname{Hom}_{k-\operatorname{Mod}}\left(A, R \otimes_{k} k^{\prime}\right)$.

Finally we prove the adjointness property. We recall from paragraph 2.1.3 (see also Remark 2.1.4) that $\left(\mathbb{O}_{k}\right.$ - Alg) is the category of $\mathbb{O}_{k}$-algebra schemes whose underlying $\mathbb{O}_{k}$-module scheme is of the form $\mathbb{V}(\mathscr{F})=\operatorname{Spec} S(\mathscr{F})$ for some quasi-coherent $\mathcal{O}_{\operatorname{Spec}(k)}$-module $\mathscr{F}$, and that $(k-\mathrm{Gr})$ is the category of affine $k$-group schemes.

2.3.2. Theorem (Adjointness property of the group algebra). The group algebra functor is left adjoint to the group of units functor. In other words, for all affine $k$-group schemes $G$ and all linear $\mathbb{O}_{k}$-algebra schemes $D$, the map that sends a morphism of algebra schemes $\mathbb{O}_{k}[G] \rightarrow D$ to the composition $G \subset \mathbb{O}_{k}[G]^{\times} \rightarrow D^{\times}$gives a bifunctorial bijection:

$$
\operatorname{Hom}_{\mathbb{O}_{k}-\operatorname{Alg}}\left(\mathbb{O}_{k}[G], D\right) \stackrel{\sim}{\rightarrow} \operatorname{Hom}_{k-G r}\left(G, D^{\times}\right) .
$$

Proof. We describe a map in the other direction and we leave to the reader the proof that it is an inverse. Let $f: G \rightarrow D^{\times}$be a morphism of $k$-group schemes. We will construct a map of functors $f^{\prime}: \mathbb{O}_{k}[G] \rightarrow D$. We know from paragraph 2.1.3 that $D=\mathbb{V}(F)$ where $F$ is a $k$-module, and that the comultiplication $\Delta_{D}: \mathrm{S}(F) \rightarrow \mathrm{S}(F) \otimes \mathrm{S}(F)$ is induced by a morphism $\Delta_{0}: F \rightarrow F \otimes F$. Consider the composition $G \rightarrow D^{\times} \subset D$ and let $g: \mathrm{S}(F) \rightarrow A$ be the corresponding map of algebras. For each $k$-algebra $R$ we have the equalities $\mathbb{O}_{k}[G](R)=\operatorname{Hom}_{k-\operatorname{Mod}}(A, R)$ and $D(R)=\operatorname{Hom}_{k-\operatorname{Mod}}(F, R)$. We define $f^{\prime}$ as follows:

$$
\begin{aligned}
\mathbb{O}_{k}[G](R) & \longrightarrow D(R) \\
(u: A \rightarrow R) & \longmapsto(u \circ g \circ i: F \rightarrow R)
\end{aligned}
$$

where $i: F \hookrightarrow S(F)$ is the inclusion as the degree 1 piece in the symmetric algebra. The map $f^{\prime}$ is a map of modules, and we only have to check that it respects the multiplication. Let $u, v: A \rightarrow R$ be module homomorphisms. We have the following commutative diagram:

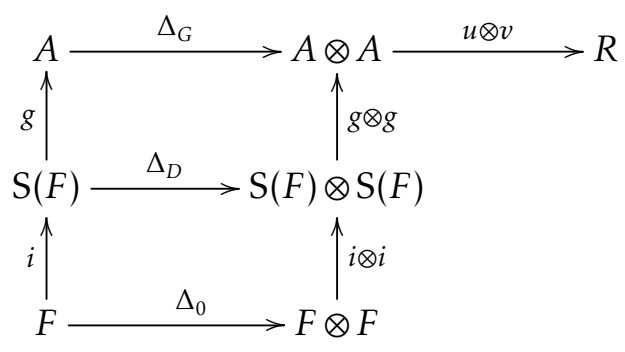


With the $\star$ notation as in Subsection 2.2, we compute:

$$
\begin{aligned}
\left(u \star_{G} v\right) \circ g \circ i & =(u \otimes v) \circ \Delta_{G} \circ g \circ i \\
& =(u g i \otimes v g i) \circ \Delta_{0} \\
& =u g i \star_{D} v g i .
\end{aligned}
$$

Thus $f^{\prime}: \mathbb{O}_{k}[G] \rightarrow D$ is a map of algebra schemes and this ends the construction.

2.3.3. Remark. It follows from this result that a smooth vector bundle with action of $G$ is the same as a (smooth) $\mathbb{O}_{k}[G]$-module scheme. Indeed, the endomorphism algebra of such a vector bundle is representable by a linear $\mathbb{O}_{k}$-algebra scheme $D$. For example, if $G$ is an affine, finite type, differentially flat $k$-group scheme, then the adjoint action on Lie $G$ makes it an $\mathbb{O}_{k}[G]$-module scheme.

\section{Weil restriction}

We keep the notations from the previous sections. In this section, we describe the Weil restriction $E=h_{*} \mathscr{G}$ of a $k[I]$-group scheme $\mathscr{G} \in \mathrm{Gr} / k[I]$ and show how it carries the structure of an object of the category of extensions $\operatorname{Ext}(I) / k$. The necessary notions of differential calculus (tangent bundle, Lie algebra, exponential) are recalled in Appendix A.

\subsection{Weil restriction of the group algebra}

If $\mathscr{G}$ is an affine $k[I]$-group scheme, its Weil restriction $h_{*} \mathscr{G}$ embeds in the pushforward algebra $h_{*} \mathbb{O}_{k[I]}[\mathscr{G}]$. (It also embeds in the algebra $\mathbb{O}_{k}\left[h_{*} \mathscr{G}\right]$ which however is less interesting in that it does not reflect the Weil restriction structure.) Our aim in this subsection is to give a description of $h_{*} \mathbb{O}_{k[I]}[\mathscr{G}]$ suited to the computation of the adjunction map $\beta \mathscr{G}$.

The starting point is the following definition and lemma. Let $A$ be a $k[I]$-algebra and $R$ a $k$-algebra. Let $v: A \rightarrow I \otimes_{k} R$ be a $k$-linear map such that there is a $k$-linear map $\bar{v}: A \rightarrow R$ satisfying $v(i x)=i \bar{v}(x)$ for all $i \in I$ and $x \in A$. Then $\bar{v}$ is uniquely determined by $v$; in fact it is already determined by the identity $v(i x)=i \bar{v}(x)$ for any fixed $i$ belonging to a basis of $I$ as a $k$-module.

3.1.1. Definition. Let $A$ be a $k[I]$-algebra and $R$ a $k$-algebra. We say that a $k$-linear map $v: A \rightarrow I \otimes_{k} R$ is $I$-compatible if there is a $k$-linear map $\bar{v}: A \rightarrow R$ such that $v(i x)=i \bar{v}(x)$ for all $i \in I$ and $x \in A$. We denote by $\operatorname{Homc}_{k}\left(A, I \otimes_{k} R\right)$ the $R$-module of $I$-compatible maps and $\operatorname{Homc}_{k}\left(A, I \otimes_{k} R\right) \rightarrow \operatorname{Hom}_{k}(A, R), v \mapsto \bar{v}$ the $R$-module map that sends $v$ to the unique map $\bar{v}$ with the properties above.

Note that since $I^{2}=0$ in $R[I]$, if $v$ is $I$-compatible then $\bar{v}$ vanishes on $I A$. In other words, the map $v \mapsto \bar{v}$ factors through $\operatorname{Hom}_{k}(A / I A, R)$.

3.1.2. Lemma. Let $A$ be a $k[I]$-algebra and $R$ a $k$-algebra.

(1) Each morphism of $k[I]$-modules $f: A \rightarrow R[I]$ is of the form $f=\bar{v}+v$ for a unique I-compatible $k$-linear map $v: A \rightarrow I \otimes_{k} R$, and conversely.

(2) Each morphism of $k[I]$-algebras $f: A \rightarrow R[I]$ is of the form $f=\bar{v}+v$ as above with $v$ satisfying moreover $v(i)=i$ for all $i \in I$ and $v(x y)=\bar{v}(x) v(y)+\bar{v}(y) v(x)$ for all $x, y \in A$, and conversely. In particular $\bar{v}: A \rightarrow R$ is a k-algebra homomorphism and $v: A \rightarrow I \otimes_{k} R$ is a $\bar{v}$-derivation.

Proof. (1) Using the decomposition $R[I]=R \oplus I \otimes_{k} R$, we can write $f(x)=u(x)+v(x)$ for some unique $k$-linear maps $u: A \rightarrow R$ and $v: A \rightarrow I \otimes_{k} R$. Then $f$ is $k[I]$-linear if and only if $f(i x)=i f(x)$ for all $i \in I$ and $x \in A$. Taking into account that $I^{2}=0$, this means that $v$ is $I$-compatible and $u=\bar{v}$. 
(2) The condition $f(1)=1$ means that $\bar{v}(1)=1$, that is $v(i)=i$ for all $i \in I$, and $v(1)=0$. The condition of multiplicativity of $f$ means that $\bar{v}$ is multiplicative and $v$ is a $\bar{v}$-derivation, i.e. $v(x y)=\bar{v}(x) v(y)+\bar{v}(y) v(x)$. In the presence of the derivation property, the multiplicativity of $\bar{v}$ is automatic (computing $v(i x y)$ in two different ways) as well as the condition $v(1)=0$ (setting $x=y=1$ ). Conversely if $v$ is $I$-compatible with $v(i)=i$ and $v(x y)=\bar{v}(x) v(y)+\bar{v}(y) v(x)$, one sees that $\bar{v}$ is a morphism of rings and $f=\bar{v}+v$ is a morphism of $k[I]$-algebras.

Now let $\mathscr{G}$ be an affine $k[I]$-group scheme. Lemma 3.1.2 shows that the Weil restriction $h_{*} \mathbb{V}(\mathscr{G} / k[I])$ can be described in terms of the scheme of $I$-compatible maps, defined as a functor on $k$-algebras by:

$$
\mathbb{O}_{\mathrm{c}}(\mathscr{G})(R):=\operatorname{Homc}_{k}\left(A, I \otimes_{k} R\right) \text {. }
$$

We know that $h_{*} \mathbb{V}(\mathscr{G} / k[I])$ supports the algebra scheme structure $h_{*} \mathbb{O}_{k[I]}[\mathscr{G}]$, and we will now identify the multiplication induced on $\mathbb{O}_{\mathrm{c}}(\mathscr{G})$ by means of this isomorphism.

3.1.3. Proposition. Let $\mathscr{G}=\operatorname{Spec}(A)$ be an affine $k[I]$-group scheme with comultiplication $\Delta: A \rightarrow A \otimes_{k[I]} A$ and counit $e: A \rightarrow k[I]$, with $e=\bar{d}+d$ for a unique I-compatible $k$-linear map $d: A \rightarrow I$.

(1) Let $R$ be a k-algebra and let $v, w: A \rightarrow I \otimes_{k} R$ be two I-compatible $k$-linear maps. Then the morphism $\bar{v} \otimes_{k} w+v \otimes_{k} \bar{w}: A \otimes_{k} A \rightarrow R$ factors through a well-defined $k$-linear morphism

$$
\bar{v} \otimes_{k} w+v \otimes_{k} \bar{w}: A \otimes_{k[I]} A \rightarrow R .
$$

(2) For $v, w$ as before let:

$$
v \diamond w:=\left(\bar{v} \otimes_{k} w+v \otimes_{k} \bar{w}\right) \circ \Delta .
$$

Then $\left(\mathbb{O}_{\mathrm{c}}(\mathscr{G}),+, \diamond\right)$ is an associative unitary $\mathbb{O}_{k}$-algebra with multiplicative unit $d$, and the map

$$
\theta_{\mathscr{G}}: \mathbb{O}_{\mathrm{c}}(\mathscr{G}) \stackrel{\sim}{\longrightarrow} h_{*} \mathbb{O}_{k[I]}[\mathscr{G}], \quad v \longmapsto \bar{v}+v
$$

is an isomorphism of associative unitary $\mathbb{O}_{k}$-algebras.

Proof. (1) The $k$-linear morphism $\bar{v} \otimes_{k} w+v \otimes_{k} \bar{w}$ takes the same value $i \bar{v}(a) \bar{w}(b)$ on the tensors $i a \otimes b$ and $a \otimes i b$ for all $i \in I, a, b \in A$. Therefore it vanishes on tensors of the form $(a \otimes b)(i \otimes 1-1 \otimes i)$. Since these tensors generate the kernel of the ring map $A \otimes_{k} A \rightarrow A \otimes_{k[I]} A$, we obtain an induced morphism $\bar{v} \otimes_{k} w+v \otimes_{k} \bar{w}: A \otimes_{k[I]} A \rightarrow R$.

(2) According to (1) the definition of $v \diamond w$ makes sense. For the rest of the statement, it is enough to prove that $\theta_{\mathscr{G}}(v \diamond w)=\theta_{\mathscr{G}}(v) \star \theta_{\mathscr{G}}(w)$ because if this is the case then all the known properties of the product $\star$ in $h_{*} \mathbb{O}_{k[I]}[\mathscr{G}]$ are transferred to $\diamond$ by the isomorphism $\theta_{\mathscr{G}}$. On one hand, using the expression $v \diamond w=\left(\bar{v} \otimes_{k} w+v \otimes_{k} \bar{w}\right) \circ \Delta$ and the fact that $\Delta$ is $k[I]$-linear, we find $\bar{v} \diamond w=\left(\bar{v} \otimes_{k} \bar{w}\right) \circ \Delta$ hence:

$$
\begin{aligned}
\theta_{\mathscr{G}}(v \diamond w) & =\left(\bar{v} \otimes_{k} \bar{w}\right) \circ \Delta+\left(\bar{v} \otimes_{k} w+v \otimes_{k} \bar{w}\right) \circ \Delta \\
& =\left[\bar{v} \otimes_{k} \bar{w}+\left(\bar{v} \otimes_{k} w+v \otimes_{k} \bar{w}\right)\right] \circ \Delta .
\end{aligned}
$$

On the other hand, we have:

$$
\theta_{\mathscr{G}}(v) \star \theta_{\mathscr{G}}(w)=\left[(\bar{v}+v) \otimes_{k}(\bar{w}+w)\right] \circ \Delta .
$$

The maps in the brackets are equal, whence $\theta_{\mathscr{G}}(v \diamond w)=\theta_{\mathscr{G}}(v) \star \theta_{\mathscr{G}}(w)$ as desired.

3.1.4. Remark. If $\left(\varepsilon_{1}, \ldots, \varepsilon_{r}\right)$ is a basis of $I$ we have a concrete description as follows. A $k$-linear map $v: A \rightarrow I \otimes_{k} R$ can be written $v=\varepsilon_{1} v_{1}+\cdots+\varepsilon_{r} v_{r}$ for some maps $v_{j}: A \rightarrow R$. Then $v$ is $I$-compatible if and only if $v_{j} \varepsilon_{i}=\delta_{i, j} \bar{v}$ for all $i, j$. If this is the case, $v_{j}$ induces a $k$-linear morphism $A \otimes_{k[I]} k\left[\varepsilon_{j}\right] \rightarrow R$ and $\bar{v}=v_{j} \varepsilon_{j}$ for each $j$. Now write $\mathscr{G}_{j}=\mathscr{G} \otimes_{k[I]} k\left[\varepsilon_{j}\right]$ and $h_{j}: \operatorname{Spec}\left(k\left[\varepsilon_{j}\right]\right) \rightarrow \operatorname{Spec}(k)$ the structure map. Also let $\mathscr{G}_{k}=\mathscr{G} \otimes_{k[I]} k$ so we have maps $\mathbb{V}\left(h_{j, !} \mathscr{G}_{j} / k\right) \rightarrow \mathbb{V}\left(\mathscr{G}_{k} / k\right), v_{j} \mapsto \bar{v}:=v_{j} \varepsilon_{j}$. Then we have an isomorphism:

$$
\mathbb{V}\left(h_{1, !} \mathscr{G}_{1} / k\right) \underset{\mathbb{V}\left(\mathscr{G}_{k} / k\right)}{\times} \cdots \underset{\mathbb{V}\left(\mathscr{G}_{k} / k\right)}{\times} \mathbb{V}\left(h_{r, !} \mathscr{G}_{r} / k\right) \stackrel{\sim}{\rightarrow} \mathbb{O}_{\mathrm{c}}(\mathscr{G})
$$

given by $\left(v_{1}, \ldots, v_{r}\right) \mapsto v=\varepsilon_{1} v_{1}+\cdots+\varepsilon_{r} v_{r}$. 


\subsection{Kernel of the adjunction $\beta: h^{*} h_{*} \mathscr{G} \rightarrow \mathscr{G}$}

Again let $\mathscr{G}=\operatorname{Spec}(A)$ be an affine $k[I]$-group scheme. Denote by $\Delta: A \rightarrow A \otimes_{k[I]} A$ the comultiplication and $e: A \rightarrow k[I]$ the counit, of the form $e=\bar{d}+d$ for a unique $I$-compatible $k$-linear map $d: A \rightarrow I$. The purpose of this subsection is to prove the following proposition.

3.2.1. Proposition. Let $\mathscr{G}$ be an affine $k[I]$-group scheme and $\mathscr{G}_{k}=i^{*} \mathscr{G}$. Let $\beta=\beta \mathscr{G}: h^{*} h_{*} \mathscr{G} \rightarrow \mathscr{G}$ be the adjunction, and $\mathrm{L}(\mathscr{G}):=\operatorname{ker}(\beta)$.

(1) Functoriality. The formation of $\mathrm{L}(\mathscr{G})$ is functorial for morphisms of pointed $k[I]$-schemes, and for morphisms of $k[I]$-group schemes.

(2) Explicit description. We have embeddings of monoids $\mathrm{L}(\mathscr{G}) \subset h^{*} h_{*} \mathscr{G} \subset\left(h^{*} \mathrm{O}_{c}(\mathscr{G}), \diamond\right)$ under which, functorially in the $k[I]$-algebra $R$ :

$$
\left(h^{*} h_{*} \mathscr{G}\right)(R)=\left\{\begin{array}{l|l}
v \in\left(h^{*} \mathbb{O}_{\mathrm{c}}(\mathscr{G})\right)(R) & \begin{array}{l}
v(i)=i \text { for all } i \in I \\
v(x y)=\bar{v}(x) v(y)+\bar{v}(y) v(x) \text { for all } x, y \in A
\end{array}
\end{array}\right\}
$$

and if $v_{R}: A \rightarrow R$ denotes the composition $A \stackrel{v}{\longrightarrow} I \otimes_{k} R \stackrel{i \otimes r \mapsto i r}{\longrightarrow} R$ :

$$
\mathrm{L}(\mathscr{G})(R)=\left\{v \in\left(h^{*} h_{*} \mathscr{G}\right)(R): \bar{v}+v_{R}=e_{R}\right\} .
$$

(3) Special fibre. There is a functorial isomorphism $i^{*} \mathrm{~L}(\mathscr{G}) \stackrel{\sim}{\rightarrow} \operatorname{Lie}\left(\mathscr{G}_{k}, I\right), v \mapsto v-d$. Under this isomorphism, the action by conjugation of $h_{*} \mathscr{G}=i^{*} h^{*} h_{*} \mathscr{G}$ on $i^{*} \mathrm{~L}(\mathscr{G})$ is given by the morphism:

$$
h_{*} \mathscr{G} \stackrel{i^{*} \beta}{\longrightarrow} \mathscr{G}_{k} \stackrel{\mathrm{Ad}}{\longrightarrow} \mathrm{GL}\left(\operatorname{Lie}\left(\mathscr{G}_{k}, I\right)\right) .
$$

(4) Case of trivial deformation groups. If $\mathscr{G}=h^{*} G$ for some affine $k$-group scheme $G$, there is a canonical and functorial isomorphism $\mathrm{L}(\mathscr{G}) \stackrel{\sim}{\rightarrow} h^{*} \operatorname{Lie}(G, I)$. More precisely, let $\exp _{G, I}: h^{*} \operatorname{Lie}(G, I) \rightarrow h^{*} G$ be the exponential morphism as defined in A.2.1. Then under the isomorphism (see A.1)

$$
\rho_{G}: \operatorname{Lie}(G, I) \times G \stackrel{\sim}{\rightarrow} \mathrm{T}(G, I),
$$

the subgroup $\mathrm{L}(\mathscr{G}) \subset h^{*} \mathrm{~T}(G, I)$ has for points the pairs $(x, g) \in h^{*} \operatorname{Lie}(G, I) \times h^{*} G$ such that $g=\exp (-x)$, and the isomorphism is given by $(x, g) \mapsto x$.

Proof. (1) If $\varphi: \mathscr{G} \rightarrow \mathscr{G}^{\prime}$ is a morphism of pointed schemes, then by functoriality of $\beta$ the morphism $h_{*} h^{*} \varphi$ takes the kernel of $\beta \mathscr{G}$ into the kernel of $\beta \mathscr{G}$ '. If moreover $\varphi$ is a map of group schemes then the restriction of $h_{*} h^{*} \varphi$ to $\mathrm{L}(\mathscr{G})$ also.

(2) In the rest of the proof we use the possibility to compute in the group algebra $\left(\mathbb{O}_{c}(\mathscr{G}),+, \diamond\right)$, see Proposition 3.1.3. The description of $h^{*} h_{*} \mathscr{G}$ as a submonoid of the multiplicative monoid of $\mathbb{O}_{\mathrm{c}}(\mathscr{G})$ is copied from Lemma 3.1.2. The description of $\mathrm{L}(\mathscr{G})$ follows from the fact that for $f \in \mathscr{G}(R[I]), f=\bar{v}+v: A \rightarrow R[I]$, the image $\beta(f) \in \mathscr{G}(R), \beta(f): A \rightarrow R$ is the map $\bar{v}+v_{R}$.

(3) - first claim. The pullback $i^{*}$ is the restriction to the category of those $k[I]$-algebras $R$ such that $I R=0$. For such an $R$, an element $v \in\left(i^{*} \mathrm{~L}(\mathscr{G})\right)(R)$ is a $k$-linear map $v: A \rightarrow R$ such that $v(i)=i$, $v(x y)=\bar{v}(x) v(y)+\bar{v}(y) v(x)$ and $\bar{v}=e_{R}$. In particular we see that $v$ is an $e_{R}$-derivation. Since also $d_{R}$ is an $e_{R}$-derivation with $d_{R}(i)=i$ for all $i \in I$, the difference $\delta:=v-d_{R}$ induces an $e_{R}$-derivation $\delta: A \rightarrow I \otimes_{k} R$ vanishing on $I A$, i.e. an $R$-point of $\operatorname{Lie}\left(\mathscr{G}_{k}, I\right)$. Conversely, any $e_{R}$-derivation $\delta: A \rightarrow I \otimes_{k} R$ vanishing on $I A$ gives rise to a $k$-linear map $v: A \rightarrow I \otimes_{k} R$ defined by $v:=d_{R}+\delta$ and satisfying the properties required to be a point of $\left(i^{*} L(\mathscr{G})\right)(R)$. Finally let $\delta_{1}, \delta_{2} \in \operatorname{Lie}\left(\mathscr{G}_{k}, I\right)(R)$. Since $\delta_{1}, \delta_{2}$ vanish on $I A$, we have $d_{R}^{*}+\delta_{1}^{*}=d_{R}^{*}+\delta_{2}^{*}=e_{R}$ and then:

$$
\left(d_{R}+\delta_{1}\right) \diamond\left(d_{R}+\delta_{2}\right)=\left[e_{R} \otimes\left(d_{R}+\delta_{2}\right)+\left(d_{R}+\delta_{1}\right) \otimes e_{R}\right] \circ \Delta
$$




$$
=\left[\left(e_{R} \otimes d_{R}+d_{R} \otimes e_{R}\right)+\left(e_{R} \otimes \delta_{2}+\delta_{1} \otimes e_{R}\right)\right] \circ \Delta .
$$

All three morphisms $e_{R} \otimes d_{R}+d_{R} \otimes e_{R}, e_{R} \otimes \delta_{2}$ and $\delta_{1} \otimes e_{R}$ factor through $A \otimes_{k[I]} A$, so the precomposition with $\Delta$ is distributive for them. Since also $d_{R}$ is the neutral element for the law $\diamond$ and $e_{R}$ is the neutral element for the law $\star$, we obtain:

$$
\begin{aligned}
\left(d_{R}+\delta_{1}\right) \diamond\left(d_{R}+\delta_{2}\right) & =\left[e_{R} \otimes d_{R}+d_{R} \otimes e_{R}\right] \circ \Delta+\left[e_{R} \otimes \delta_{2}\right] \circ \Delta+\left[\delta_{1} \otimes e_{R}\right] \circ \Delta \\
& =d_{R}+\delta_{1}+\delta_{2} .
\end{aligned}
$$

This shows that the isomorphism $i^{*} \mathrm{~L}(\mathscr{G}) \stackrel{\sim}{\rightarrow} \operatorname{Lie}\left(\mathscr{G}_{k}\right), v \mapsto v-d_{R}$ is a morphism of groups.

(3) - second claim. Let $R$ be a $k[I]$-algebra such that $I R=0$. The action of an element $u \in\left(h^{*} h_{*} \mathscr{G}\right)(R)$ on an element $v=d_{R}+\delta \in \mathrm{L}(\mathscr{G})(R)$ by conjugation can be computed in the algebra $h^{*} \mathbb{O}_{\mathrm{c}}(\mathscr{G})$ :

$$
u \diamond v \diamond u^{-1}=u \diamond\left(d_{R}+\delta\right) \diamond u^{-1}=u \diamond d_{R} \diamond u^{-1}+u \diamond \delta \diamond u^{-1}=d_{R}+u \diamond \delta \diamond u^{-1} .
$$

We see that $u$ is acting on $\delta$ by conjugation in the group algebra. This is the adjoint action, as explained in Proposition A.3.1.

(4) When $\mathscr{G}=h^{*} G$, the adjunction map is the infinitesimal translation as in Proposition A.2.2(3). The $R$-points of $\mathrm{L}(\mathscr{G})$ are the pairs $(x, g)$ such that $\exp (x) g=1$ in $G(R)$. This amounts to $g=\exp (-x)$ which proves (4).

\subsection{Extension structure of the Weil restriction}

Let $\mathscr{G}$ be a $k[I]$-group scheme. The notion of rigidification for $\mathscr{G}$ and the property that $\mathscr{G}$ be rigid are defined in 1.1.2. Here are some remarks.

(1) If $\sigma: h^{*} \mathscr{G}_{k} \stackrel{\sim}{\rightarrow} \mathscr{G}$ is a rigidification, then $\sigma(1)^{-1} \cdot \sigma$ is another. Therefore if there exists a rigidification $\sigma$, we may always assume moreover that $\sigma(1)=1$.

(2) By the infinitesimal lifting criterion, all smooth affine $k[I]$-group schemes are rigid. By Cartier duality, $k[I]$-group schemes of multiplicative type are rigid.

(3) If $\alpha: \mathscr{G} \rightarrow \mathscr{G}^{\prime}$ is a morphism between rigid $k[I]$-group schemes, it is not always possible to choose rigidifications for $\mathscr{G}$ and $\mathscr{G}^{\prime}$ that are compatible in the sense that $\sigma^{\prime} \circ h^{*} \alpha=\alpha \circ \sigma$. For instance let $I=k \varepsilon$ and let $\alpha: \mathbb{G}_{a} \rightarrow \mathbb{G}_{a}$ be the morphism defined by $\alpha(x)=\varepsilon x$. Then $\mathscr{G}$ and $\mathscr{G}^{\prime}$ are rigid but since $h^{*} \alpha=0$, there do not exist compatible rigidifications.

3.3.1. Lemma. Let $\mathscr{G}$ be a $k[I]$-group scheme such that the restriction homomorphism $\mathscr{G}_{k}=i * \mathscr{G}$ is $k$-flat. Let $\pi:=i^{*} \beta \mathscr{G}: h_{*} \mathscr{G} \rightarrow \mathscr{G}_{k}$. Then the adjunction $\mathrm{Hom}_{\mathrm{Sch} / k[I]}\left(h^{*} \mathscr{G}_{k}, \mathscr{G}\right)=\mathrm{Hom}_{\mathrm{Sch} / k}\left(\mathscr{G}_{k}, h_{*} \mathscr{G}\right)$ induces a bijection between rigidifications of $\mathscr{G}$ and sections of $\pi$.

Proof. Let $\sigma: h^{*} \mathscr{G}_{k} \stackrel{\sim}{\rightarrow} \mathscr{G}$ be a rigidification and $s:=h_{*} \sigma \circ \alpha \mathscr{G}_{k}$. Then we have $\sigma=\beta \mathscr{G} \circ h^{*} s$ and by applying $i^{*}$ we find id $\mathscr{G}_{k}=i^{*} \sigma=\pi \circ s$ hence $s$ is a section of $\pi$. Conversely let $s$ be a section of $\pi$ and $\sigma:=\beta \mathscr{G} \circ h^{*} s$. Then $i^{*} \sigma=i^{*} \beta \mathscr{G} \circ i^{*} h^{*} s=\pi \circ s=\mathrm{id}_{\mathscr{G}_{k}}$ hence $\sigma$ lifts the identity. In particular $\sigma$ is an affine morphism. Since moreover $h^{*} \mathscr{C}_{k}$ is flat, we conclude that $\sigma$ is an isomorphism, hence a rigidification.

3.3.2. Lemma. Let $\mathscr{G}$ be an affine, differentially flat and rigid $k[I]$-group scheme. Then $\beta: h^{*} h_{*} \mathscr{G} \rightarrow \mathscr{G}$ is faithfully flat and we have an exact sequence:

$$
1 \longrightarrow \mathrm{L}(\mathscr{G}) \longrightarrow h^{*} h_{*} \mathscr{G} \stackrel{\beta}{\longrightarrow} \mathscr{G} \longrightarrow 1
$$

If $\mathscr{G}$ is of finite type over $k[I]$, then $\beta$ is smooth. 
Proof. Again we put $\pi=i^{*} \beta: h_{*} \mathscr{G} \rightarrow \mathscr{G}_{k}$. Let $\sigma: h^{*} \mathscr{G}_{k} \stackrel{\sim}{\rightarrow} \mathscr{G}$ be a rigidification and $s: \mathscr{G}_{k} \rightarrow h_{*} \mathscr{G}$ the corresponding section of $\pi$, see Lemma 3.3.1. By Proposition 3.2.1(3) the kernel of $\pi$ is $\operatorname{Lie}\left(\mathscr{G}_{k}\right)$ which is $k$-flat. The section $s$ provides an isomorphism of $k$-schemes $\operatorname{Lie}\left(\mathscr{G}_{k}\right) \times \mathscr{G}_{k} \simeq h_{*} \mathscr{G}$ which shows that $h_{*} \mathscr{G}$ is $k$-flat so that $h^{*} h_{*} \mathscr{G}$ is $k[I]$-flat. It follows also that $\pi: h_{*} \mathscr{G} \rightarrow \mathscr{G}_{k}$ is faithfully flat and by the "critère de platitude par fibres" in the nilpotent case ([SP, Tag 06A5]) we deduce that the morphism $\beta$ is faithfully flat. Finally if $\mathscr{G}$ is of finite type over $k[I]$, then the special fibre of $\mathrm{L}(\mathscr{G})$ is the smooth vector group $\operatorname{Lie}\left(\mathscr{G}_{k}\right)$, hence $\mathrm{L}(\mathscr{G})$ is smooth and so is $\beta$.

3.3.3. Example. Here is an example where the result above fails, for a non-rigid group. Assume $k$ is a field of characteristic $p>0$. Let $I=k \varepsilon$ be free of rank 1 . Let $\mathscr{G}$ be the kernel of the endomorphism $\mathbb{G}_{a} \rightarrow \mathbb{G}_{a}, x \mapsto x^{p}-\varepsilon x$. Then $\left(h^{*} h_{*} \mathscr{G}\right)(R)$ is the set of elements $a \oplus b \varepsilon \in R \oplus R \varepsilon$ such that $(a \oplus b \varepsilon)^{p}=\varepsilon(a \oplus b \varepsilon)$. This equation is equivalent to $a^{p}=\varepsilon a$, hence $a=0$. Thus $(a, b) \mapsto b$ is an isomorphism $h^{*} h_{*} \mathscr{G} \stackrel{\sim}{\longrightarrow} \mathbb{G}_{a, k[\varepsilon]}$. The map $\left(h^{*} h_{*} \mathscr{G}\right)(R) \rightarrow \mathscr{G}(R)$ sends $b$ to $b \varepsilon_{R}$. In other words, if we let $K_{\varepsilon} \simeq \operatorname{Spec}(k[\varepsilon][x] /(\varepsilon x))$ denote the kernel of $\varepsilon: \mathbb{G}_{a, k[\varepsilon]} \rightarrow \mathbb{G}_{a, k[\varepsilon]}$, then the sequence of the lemma is $1 \rightarrow K_{\varepsilon} \rightarrow \mathbb{G}_{a, k[\varepsilon]} \stackrel{\varepsilon}{\longrightarrow} \mathscr{G} \rightarrow 1$. Here, the map $\varepsilon: \mathbb{G}_{a, k[\varepsilon]} \rightarrow \mathscr{G}$ is not flat so this is not an exact sequence of flat group schemes.

3.3.4. Proposition. For each $\mathscr{G} \in \mathrm{Gr} / k[I]$, the restriction via $i^{*}$ of the exact sequence of Lemma 3.3 .2 gives $E:=h_{*} G$ the structure of an object of $\operatorname{Ext}(I) / k$. Hence Weil restriction gives a functor:

$$
h_{*}: \mathrm{Gr} / k[I] \rightarrow \operatorname{Ext}(I) / k \text {. }
$$

Proof. From Lemma 3.3.2 we have an exact sequence $1 \rightarrow \mathrm{L}(\mathscr{G}) \rightarrow h^{*} h_{*} \mathscr{G} \rightarrow \mathscr{G} \rightarrow 1$. It follows from point (2) in Proposition 3.2.1 that when we restrict to the closed fibre, we obtain an exact sequence:

$$
1 \longrightarrow \operatorname{Lie}\left(\mathscr{G}_{k}, I\right) \longrightarrow E \longrightarrow \mathscr{G}_{k} \longrightarrow 1
$$

where the $\mathscr{G}_{k}$-action on $\operatorname{Lie}\left(\mathscr{G}_{k}\right)$ induced by the extension is the adjoint representation. The same reference proves that this extension is functorial in $\mathscr{G}$. More precisely, if $u: \mathscr{G} \rightarrow \mathscr{G}^{\prime}$ is a morphism of affine, differentially flat, rigid $k[I]$-group schemes, then we obtain a morphism between the extensions $E=h_{*} \mathscr{G}$ and $E^{\prime}=h_{*} \mathscr{G}^{\prime}$ as follows:

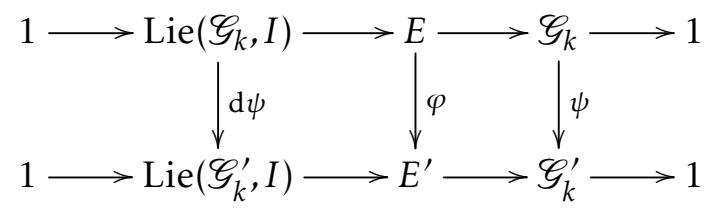

where $\varphi=h_{*} u$ and $\psi=u_{k}=i^{*} u$, the restriction of $u$ along $i: \operatorname{Spec}(k) \hookrightarrow \operatorname{Spec}(k[I])$.

We draw a corollary that will be useful in Section 5 .

3.3.5. Corollary. Let $Y$ be an affine, flat, rigid $k[I]$-scheme and $\mathbb{V}(Y)=\mathbb{V}(Y / k[I]), \mathbb{V}\left(Y_{k}\right)=\mathbb{V}\left(Y_{k} / k\right)$ the vector bundle envelopes. Then we have a split exact sequence of flat $k$-group schemes:

$$
0 \longrightarrow \mathbb{V}\left(Y_{k}\right) \otimes_{\mathbb{O}_{k}} \mathbb{V}\left(I^{\vee}\right) \longrightarrow h_{*} \mathbb{V}(Y) \stackrel{\pi}{\longrightarrow} \mathbb{V}\left(Y_{k}\right) \longrightarrow 0 .
$$

Moreover $h_{*} \mathbb{V}(Y)$ is flat as an $\mathbb{O}_{k}[I]$-module scheme and the surjection $\pi$ is isomorphic to the map given by reduction modulo $I$.

Proof. Recall from paragraph 2.1.2 that $\mathbb{O}_{k}[I]:=h_{*} \mathbb{O}_{k[I]}$. By the assumptions on $Y$, the vector bundle $\mathbb{V}(Y)$ is affine, differentially flat and rigid over $k[I]$. Thus Proposition 3.3.4 yields the displayed exact sequence. Using a rigidification for $Y$ and base change for the functor $\mathbb{V}$, we have $h_{*} \mathbb{V}(Y) \simeq h_{*} \mathbb{V}\left(h^{*} Y_{k}\right) \simeq h_{*} h^{*} \mathbb{V}\left(Y_{k}\right)$. Like in Proposition 2.3.1(4), we have an isomorphism of $\mathbb{O}_{k}$-modules $\mathbb{V}\left(Y_{k}\right) \otimes_{\mathbb{O}_{k}} \mathbb{O}_{k}[I] \stackrel{\sim}{\rightarrow} h_{*} h^{*} \mathbb{V}\left(Y_{k}\right)$ defined by $x \otimes a \mapsto a x$. Given that $\mathbb{V}\left(Y_{k}\right)$ is $\mathbb{O}_{k}$-flat, this proves that $h_{*} \mathbb{V}(Y)$ is $\mathbb{O}_{k}[I]$-flat. Even more, we have an isomorphism $h_{*} \mathbb{V}(Y) \simeq \mathbb{V}\left(Y_{k}\right) \oplus\left(\mathbb{V}\left(Y_{k}\right) \otimes_{\mathbb{O}_{k}} \mathbb{V}\left(I^{\vee}\right)\right)$ and $\pi$ is the projection onto the first factor, i.e. the map given by reduction modulo $I$. 


\section{Weil extension}

In this section, we construct a functor $h^{+}$called Weil extension which is a quasi-inverse to the functor $h_{*}$ of Weil restriction described in the previous section. The idea behind the construction is that one can recover a $k[I]$-group scheme $\mathscr{G}$ from the extension $E=h_{*} \mathscr{G}$ by looking at the target of the adjunction $\beta_{\mathscr{G}}: h^{*} E=h^{*} h_{*} \mathscr{G} \rightarrow \mathscr{G}$. In turn, in order to reconstruct the faithfully flat morphism $\beta \mathscr{G}$ it is enough to know its kernel $K$. In the case where $\mathscr{G}$ is a constant group $h^{*} G$, which in other words is the case where $E$ is a tangent bundle $\mathrm{T}(G, I)$, Proposition 3.2.1(4) hints the correct expression $K=\left\{(x, g) \in h^{*} E ; g=\exp (-x)\right\}$. The definition of $K$ for general extensions $1 \rightarrow \operatorname{Lie}(G, I) \rightarrow E \rightarrow G \rightarrow 1$ where $G$ is an affine, differentially flat $k$-group scheme, builds on this intuition.

\subsection{Hochschild extensions}

The construction of an extension from a 2-cocycle is well-known; we recall it to set up the notations. Recall from [DG70, chap. II, $\S 3$, no 2] that if $G$ is a $k$-group functor and $M$ is a $k$-G-module functor, then a Hochschild extension or simply $H$-extension of $G$ by $M$ is an exact sequence of group functors

$$
1 \longrightarrow M \stackrel{i}{\longrightarrow} E \stackrel{\pi}{\longrightarrow} G
$$

such that $\pi$ has a section (which is not required to be a morphism of groups). From a given section $s: G \rightarrow E$, we can produce a unique morphism $c: G \times G \rightarrow M$ such that $i\left(c\left(g, g^{\prime}\right)\right):=s(g) s\left(g^{\prime}\right) s\left(g g^{\prime}\right)^{-1}$. This is a 2-cocycle, i.e. it satisfies the identity

$$
c\left(g, g^{\prime}\right)+c\left(g g^{\prime}, g^{\prime \prime}\right)=g \cdot c\left(g^{\prime}, g^{\prime \prime}\right)+c\left(g, g^{\prime} g^{\prime \prime}\right) .
$$

Note that we may always replace $s$ by the section $G \rightarrow E, g \mapsto s(1)^{-1} s(g)$ to obtain a section such that $s(1)=1$. When this is the case, we have $c(g, 1)=c\left(1, g^{\prime}\right)=0$ for all $g, g^{\prime}$ and we say that $c$ is normalized. Conversely, starting from a cocycle $c$, the functor $E_{c}=M \times G$ with multiplication defined by

$$
(x, g) \cdot\left(x^{\prime}, g^{\prime}\right):=\left(x+g \cdot x^{\prime}+c\left(g, g^{\prime}\right), g g^{\prime}\right)
$$

is an $H$-extension. The map $s: G \rightarrow E_{c}, g \mapsto(0, g)$ is a possible choice of section for $\pi$. It follows from the previous comments that we may always change the cocycle into a normalized cocycle.

\subsection{Kernel of the adjunction, reprise}

In this subsection, we prepare the construction of the kernel of the adjunction map $\beta_{h^{+} E}$ of the (yet to be produced) Weil extension $h^{+} E$. The end result is in Proposition 4.3.1 of the next subsection. Note that in spite of the similarity of titles, the viewpoint is different from that of Subsection 3.2.

Let $G$ be an affine $k$-group scheme, and $\operatorname{Lie}(G, I)$ its Lie algebra relative to $I$, viewed as an affine $k$-group scheme with the adjoint action of $G$. To any 2-cocycle $c: G \times G \longrightarrow \operatorname{Lie}(G, I)$ we attach as before an $H$-extension $E_{c}=\operatorname{Lie}(G, I) \times G$ with multiplication:

$$
(x, g) \cdot\left(x^{\prime}, g^{\prime}\right):=\left(x+\operatorname{Ad}(g) x^{\prime}+c\left(g, g^{\prime}\right), g g^{\prime}\right) .
$$

Our group $E_{c}$ has a structure of $H$-extension:

$$
1 \longrightarrow \operatorname{Lie}(G, I) \stackrel{x \mapsto(x, 1)}{\longrightarrow} E_{c} \stackrel{(x, g) \mapsto g}{\longrightarrow} G \longrightarrow 1 .
$$

The following result is the heart of the construction of the Weil extension functor $h^{+}$. We point out that among the groups $K_{\lambda}\left(E_{c}\right)$ introduced here, it is especially $K_{-1}\left(E_{c}\right)$ that will be relevant in the sequel, as Proposition 3.2.1(4) shows. However, we include the whole family $K_{\lambda}\left(E_{c}\right)$ since it comes with no extra cost and brings interesting insight, in the sense that it ultimately provides an explicit linear path in the $\mathbb{O}_{k}$-module stack $\mathrm{Gr} / k[I]$. 
4.2.1. Proposition. Let $h: \operatorname{Spec}(k[I]) \rightarrow \operatorname{Spec}(k)$ be the $k$-scheme of dual numbers. Fix $\lambda \in k$.

(1) Let $G$ be an affine $k$-group scheme and let $E_{c}$ be the $H$-extension constructed out of a normalized 2-cocycle $c: G \times G \longrightarrow \operatorname{Lie}(G, I)$. Let $K_{\lambda}\left(E_{c}\right) \subset h^{*} E_{c}$ be the subfunctor defined by:

$$
K_{\lambda}\left(E_{c}\right)=\left\{(x, g) \in h^{*} E_{c} ; g=\exp (\lambda x)\right\} .
$$

Then $K_{\lambda}\left(E_{c}\right)$ is a closed normal sub-k[I]-group scheme of $h^{*} E_{c}$.

(2) Let $G, G^{\prime}$ be affine $k$-group schemes and $E_{c}, E_{c^{\prime}}$ be the $H$-extensions constructed out of some chosen normalized 2-cocycles c, $c^{\prime}$. Let $f: E_{c} \rightarrow E_{c^{\prime}}$ be a morphism of extensions:

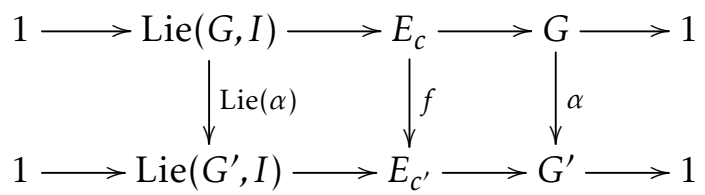

Then $\left(h^{*} f\right)\left(K_{\lambda}\left(E_{c}\right)\right) \subset K_{\lambda}\left(E_{c^{\prime}}\right)$, with equality if $f$ is an isomorphism.

When the extension $E_{c}$ is clear from context, we write $K_{\lambda}$ instead of $K_{\lambda}\left(E_{c}\right)$. We will prove the proposition after a few preliminaries. First of all, for the convenience of the reader, we recall the description of morphisms of extensions, in the abstract group setting for simplicity.

4.2.2. Lemma. Let $\alpha: G \rightarrow G^{\prime}$ be a morphism of groups and $\delta: L \rightarrow L^{\prime}$ be a morphism from a $G$-module to a $G^{\prime}$-module which is $\alpha$-equivariant. Let $E \in \mathcal{E} x t(G, L)$ and $E^{\prime} \in \mathcal{E} x t\left(G^{\prime}, L^{\prime}\right)$ be two extensions.

(1) There exists a morphism of extensions $f: E \rightarrow E^{\prime}$, i.e. a diagram

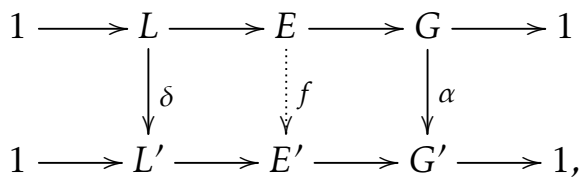

if and only if $\alpha^{*}\left[E^{\prime}\right]=\delta_{*}[E]$ in $\mathrm{H}^{2}\left(G, L^{\prime}\right)$, and if this condition holds then the set of morphisms is a principal homogeneous space under the set of 1-cocycles $Z^{1}\left(G, L^{\prime}\right)$. More precisely, assume that we describe $E$ with a normalized cocycle $c: G \times G \rightarrow L$ so that $E \simeq L \times G$ with multiplication $(x, g) \cdot\left(x^{\prime}, g^{\prime}\right)=\left(x+g \cdot x^{\prime}+c\left(g, g^{\prime}\right), g g^{\prime}\right)$, and we describe $E^{\prime}$ similarly with a normalized cocycle $c^{\prime}$. Then all morphisms $f: E \rightarrow E^{\prime}$ are of the form $f(x, g)=(\delta(x)+\varphi(g), \alpha(g))$ for a unique 1-cochain $\varphi: G \rightarrow L^{\prime}$ such that $\partial \varphi=c^{\prime} \circ \alpha-\delta \circ c$.

(2) If $E, E^{\prime}$ are two extensions of $G$ by $L$, then the set of morphisms of extensions $E \rightarrow E^{\prime}$ is a principal homogeneous space under the group $Z^{1}(G, L)$, more precisely all morphisms are of the form $f(x, g)=(x+\varphi(g), g)$ for a unique $\varphi \in \mathrm{Z}^{1}(G, L)$. All of them are isomorphisms.

(3) Assume that the extension is trivial, so that $[E]=0 \in \mathrm{H}^{2}(G, L)$. Then all group sections $G \rightarrow E$ of the extension are of the form $s(x, g)=(\varphi(g), g)$ for a unique $\varphi \in Z^{1}(G, L)$ such that $\partial \varphi=c$.

Proof. Any morphism of extensions can be written as $f: L \times G \rightarrow L^{\prime} \times G^{\prime},(x, g) \mapsto(u(x, g), \alpha(g))$ with $u(x, 1)=\delta(x)$. The property that $f$ is a morphism of groups translates into the identity:

$$
u\left(x_{1}+g_{1} \cdot x_{2}+c\left(g_{1}, g_{2}\right), g_{1} g_{2}\right)=u\left(x_{1}, g_{1}\right)+\alpha\left(g_{1}\right) \cdot u\left(x_{2}, g_{2}\right)+c^{\prime}\left(\alpha g_{1}, \alpha g_{2}\right) .
$$

Setting $x_{1}=x, x_{2}=0, g_{1}=1, g_{2}=g$, and $\varphi(g):=u(0, g)$ we find $u(x, g)=\delta(x)+\varphi(g)$ for all $x, g$. The above identity implies $\varphi\left(g_{1} g_{2}\right)-\varphi\left(g_{1}\right)-\alpha\left(g_{1}\right) \cdot \varphi\left(g_{2}\right)=c^{\prime}\left(\alpha g_{1}, \alpha g_{2}\right)-\delta\left(c\left(g_{1}, g_{2}\right)\right)$. This means that $\partial \varphi=c^{\prime} \circ \alpha-\delta \circ c$ as claimed in (1). Considering the particular case of morphisms

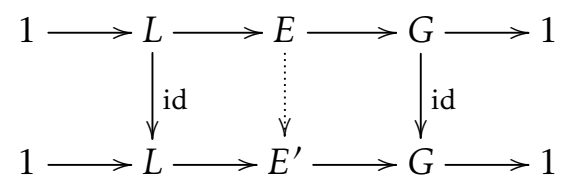


we get (2), and considering the case of morphisms

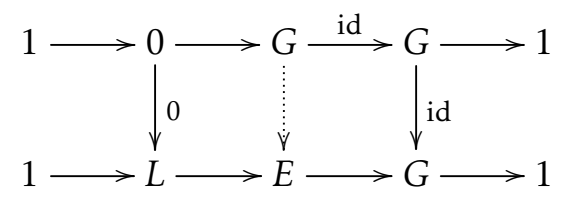

we get (3).

We come back to the extension $E_{c}$. The lemma tells us that the group Aut ext $\left(E_{c}\right)$ of automorphisms of $E_{c}$ as an extension is isomorphic to the group of 1-cocycles $Z^{1}(G, \operatorname{Lie}(G, I))$. Item (2) of Proposition 4.2.1 says in particular that $K_{\lambda}\left(E_{c}\right)$ is stable under these particular automorphisms.

Now we record a few technical properties concerning the exponential and the cocycles. For simplicity we write exp instead of $\exp _{G}$.

4.2.3. Lemma. Let $G$ be an affine $k$-group scheme and $c: G \times G \longrightarrow \operatorname{Lie}(G, I)$ a normalized 2-cocycle. Let $\exp : h^{*} \operatorname{Lie}(G, I) \rightarrow h^{*} G$ be the exponential morphism as defined in Subsection A.2. Let $R$ be a $k[I]$-algebra and $g, g^{\prime}, g^{\prime \prime} \in G(R)$. Assume that $g$ is an exponential i.e. an element in the image of exp. Then, we have:

(1) $\exp \left(c\left(g, g^{\prime}\right)\right)=\exp \left(c\left(g^{\prime}, g\right)\right)=1$,

(2) $\exp \left(c\left(g g^{\prime}, g^{\prime \prime}\right)\right)=\exp \left(c\left(g^{\prime} g, g^{\prime \prime}\right)\right)=\exp \left(c\left(g^{\prime}, g g^{\prime \prime}\right)\right)=\exp \left(c\left(g^{\prime}, g^{\prime \prime} g\right)\right)=\exp \left(c\left(g^{\prime}, g^{\prime \prime}\right)\right)$.

The same statements hold with $c$ replaced by $\lambda c$, for each $\lambda \in k$.

Proof. (1) Apply Lemma A.2.3 to the morphism of pointed schemes $\phi=c\left(-, g^{\prime}\right): G \otimes_{k} R \rightarrow(\operatorname{Lie}(G, I)) \otimes_{k} R$ to obtain $\exp \left(c\left(g, g^{\prime}\right)\right)=1$. Similarly $\exp \left(c\left(g^{\prime}, g\right)\right)=1$.

(2) Write $g=\exp (x)$. Since $\operatorname{Lie}(G, I)=\operatorname{Lie}(G) \otimes \mathbb{V}\left(I^{\vee}\right)$ we can write $x$ as a sum of tensors $y \otimes i$. Working inductively on the number of tensors in the sum, we can assume that $x=y \otimes i$. We prove successively that each of the first four terms equals $\exp \left(c\left(g^{\prime}, g^{\prime \prime}\right)\right)$.

a. The cocycle identity $c\left(g, g^{\prime}\right)+c\left(g g^{\prime}, g^{\prime \prime}\right)=\operatorname{Ad}(g) c\left(g^{\prime}, g^{\prime \prime}\right)+c\left(g, g^{\prime} g^{\prime \prime}\right)$ together with (1) imply $\exp \left(c\left(g g^{\prime}, g^{\prime \prime}\right)\right)=\exp \left(\operatorname{Ad}(g) c\left(g^{\prime}, g^{\prime \prime}\right)\right)$. Since $g$ is an exponential, according to Proposition A.2.2(4) its adjoint action is given by $\operatorname{Ad}(g) c\left(g^{\prime}, g^{\prime \prime}\right)=c\left(g^{\prime}, g^{\prime \prime}\right)+i\left[x, c\left(g^{\prime}, g^{\prime \prime}\right)\right]$. Since multiples of $i$ lie in the kernel of exp, see Proposition A.2.2(6), we deduce $\exp \left(\operatorname{Ad}(g) c\left(g^{\prime}, g^{\prime \prime}\right)\right)=\exp \left(c\left(g^{\prime}, g^{\prime \prime}\right)\right)$.

b. Since $\gamma:=g^{\prime} g\left(g^{\prime}\right)^{-1}$ is an exponential, $\exp \left(c\left(g^{\prime} g, g^{\prime \prime}\right)\right)=\exp \left(c\left(\gamma g^{\prime}, g^{\prime \prime}\right)\right)=\exp \left(c\left(g^{\prime}, g^{\prime \prime}\right)\right)$ by a.

c. The cocycle identity with $g$ and $g^{\prime}$ exchanged reads $c\left(g^{\prime}, g\right)+c\left(g^{\prime} g, g^{\prime \prime}\right)=\operatorname{Ad}\left(g^{\prime}\right) c\left(g, g^{\prime \prime}\right)+c\left(g^{\prime}, g g^{\prime \prime}\right)$. We deduce $\exp \left(c\left(g^{\prime} g, g^{\prime \prime}\right)\right)=\exp \left(c\left(g^{\prime}, g g^{\prime \prime}\right)\right)$. We conclude with $\mathrm{b}$.

d. Again this follows from the fact that $g^{\prime \prime} g\left(g^{\prime \prime}\right)^{-1}$ is an exponential.

The final claim of the lemma holds because $\lambda c$ is again a normalized cocycle.

Proof of Proposition 4.2.1. Let us write $K=K_{\lambda}\left(E_{c}\right)$ for simplicity. Obviously $K$ is a closed subfunctor of $h^{*} E_{c}$ which is isomorphic to $h^{*} \operatorname{Lie}(G, I)$ as a $k[I]$-scheme. For the verification of points (1) and (2) we let $R$ be an arbitrary $k[I]$-algebra.

(1) First, let us prove that $K$ is a subgroup scheme. Let $(x, g)$ and $\left(x^{\prime}, g^{\prime}\right)$ be two $R$-valued points of $K$ so we have $g=\exp (\lambda x)$ and $g^{\prime}=\exp \left(\lambda x^{\prime}\right)$. On one hand, using Proposition A.2.2(4) we see that $\operatorname{Ad}(g) x^{\prime}=x^{\prime}+\varepsilon\left[x, x^{\prime}\right]$ and by Proposition A.2.2(6) we deduce $\exp \left(\lambda \operatorname{Ad}(g) x^{\prime}\right)=\exp \left(\lambda x^{\prime}\right)$. On the other hand, by Lemma 4.2.3(1) we have $\exp \left(\lambda c\left(g, g^{\prime}\right)\right)=1$. Putting all this together we get:

$$
\exp \left(\lambda x+\lambda \operatorname{Ad}(g) x^{\prime}+\lambda c\left(g, g^{\prime}\right)\right)=\exp (\lambda x) \exp \left(\lambda \operatorname{Ad}(g) x^{\prime}\right) \exp \left(\lambda c\left(g, g^{\prime}\right)\right)
$$




$$
\begin{aligned}
& =\exp (\lambda x) \exp \left(\lambda x^{\prime}\right) \\
& =g g^{\prime}
\end{aligned}
$$

This proves that the product $(x, g) \cdot\left(x^{\prime}, g^{\prime}\right)$ is a point of $K$. Using the same arguments we prove that the inverse $(x, g)^{-1}=\left(-\operatorname{Ad}\left(g^{-1}\right) x-c\left(g^{-1}, g\right), g^{-1}\right)$ is a point of $K$. Hence $K$ is a subgroup scheme.

Second, let us prove that $K$ is stable by inner automorphisms. Let $(x, g)$ and $\left(x^{\prime}, g^{\prime}\right)$ be $R$-valued points of $h^{*} E_{c}$ and $K$ respectively. We must prove that $\left(x^{\prime \prime}, g^{\prime \prime}\right):=(x, g) \cdot\left(x^{\prime}, g^{\prime}\right) \cdot(x, g)^{-1}$ lies in $K$. Writing $x^{\prime}$ as a sum of tensors $x_{s}=y_{s} \otimes i_{s}$ and setting $g_{s}=\exp \left(\lambda x_{s}\right)$, we have $\left(x_{s}, g_{s}\right) \in K(R)$ and since $K$ is a subgroup scheme, it is enough to prove that $(x, g) \cdot\left(x_{s}, g_{s}\right) \cdot(x, g)^{-1}$ lies in $K$. In other words, we may and do assume in the sequel that $x^{\prime}=y \otimes i$. We first consider $\left(x_{1}, g_{1}\right):=\left(x^{\prime}, g^{\prime}\right) \cdot(x, g)^{-1}$. Using the fact that $g^{\prime}=\exp \left(\lambda x^{\prime}\right)$ and Proposition A.2.2(4), we find

$$
\operatorname{Ad}\left(g^{\prime}\right)\left(-\operatorname{Ad}\left(g^{-1}\right) x-c\left(g^{-1}, g\right)\right)=-\operatorname{Ad}\left(g^{-1}\right) x-c\left(g^{-1}, g\right)+b
$$

where $b \in I \cdot \operatorname{Lie}(G, I)(R)$ is a certain bracket, and hence:

$$
\begin{aligned}
\left(x_{1}, g_{1}\right) & =\left(x^{\prime}, g^{\prime}\right) \cdot\left(-\operatorname{Ad}\left(g^{-1}\right) x-c\left(g^{-1}, g\right), g^{-1}\right) \\
& =\left(x^{\prime}-\operatorname{Ad}\left(g^{-1}\right) x-c\left(g^{-1}, g\right)+b+c\left(g^{\prime}, g^{-1}\right), g^{\prime} g^{-1}\right) .
\end{aligned}
$$

Now $\left(x^{\prime \prime}, g^{\prime \prime}\right)=(x, g) \cdot\left(x_{1}, g_{1}\right)=\left(x+\operatorname{Ad}(g) x_{1}+c\left(g, g_{1}\right), g g_{1}\right)$ and our task is to check that

$$
\exp \left(\lambda x+\lambda \operatorname{Ad}(g) x_{1}+\lambda c\left(g, g_{1}\right)\right)=g g_{1} .
$$

We note the following:

a. We have: $\lambda x+\lambda \operatorname{Ad}(g) x_{1}=\lambda \operatorname{Ad}(g) x^{\prime}-\lambda \operatorname{Ad}(g) c\left(g^{-1}, g\right)+b+\lambda \operatorname{Ad}(g) c\left(g^{\prime}, g^{-1}\right)$. By Proposition A.2.2(6), the term $b$ will disappear upon taking exponentials, so we may disregard it. Similarly, by Lemma 4.2.3(1) the exponential of $\lambda \operatorname{Ad}(g) c\left(g^{\prime}, g^{-1}\right)$ equals 1. Also, using the cocycle relation we see that $\operatorname{Ad}(g) c\left(g^{-1}, g\right)=c\left(g, g^{-1}\right)$. Hence:

$$
\exp \left(\lambda x+\lambda \operatorname{Ad}(g) x_{1}\right)=\exp \left(\lambda \operatorname{Ad}(g) x^{\prime}-\lambda c\left(g, g^{-1}\right)\right)
$$

b. By Lemma 4.2.3(2) we have $\exp \left(\lambda c\left(g, g_{1}\right)\right)=\exp \left(\lambda c\left(g, g^{\prime} g^{-1}\right)\right)=\exp \left(\lambda c\left(g, g^{-1}\right)\right)$.

c. Using Proposition A.2.2(3) we have $g g_{1}=g g^{\prime} g^{-1}=g \exp \left(\lambda x^{\prime}\right) g^{-1}=\exp \left(\lambda \operatorname{Ad}(g) x^{\prime}\right)$.

Putting a-b-c together we get $\exp \left(\lambda x+\lambda \operatorname{Ad}(g) x_{1}+\lambda c\left(g, g_{1}\right)\right)=\exp \left(\lambda \operatorname{Ad}(g) x^{\prime}\right)=g g_{1}$ as desired.

(2) Let us write $K=K_{\lambda}\left(E_{c}\right)$ and $K^{\prime}=K_{\lambda}\left(E_{c^{\prime}}\right)$ for simplicity. According to Lemma 4.2.2, any morphism of extensions $f: E_{c} \rightarrow E_{c^{\prime}}$ is of the form $f(x, g)=(\operatorname{Lie}(\alpha)(x)+\varphi(g), \alpha(g))$ for a unique $\varphi: G \rightarrow \operatorname{Lie} G^{\prime}$ satisfying $\varphi\left(g g^{\prime}\right)-\varphi(g)-\operatorname{Ad}(g) \varphi\left(g^{\prime}\right)=c^{\prime}\left(\alpha g, \alpha g^{\prime}\right)-\operatorname{Lie}(\alpha)\left(c\left(g, g^{\prime}\right)\right)$. Setting $g=g^{\prime}=1$ we see that such a $\varphi$, hence also $\lambda \varphi$, is a map of pointed schemes. This being said, if $(x, g)$ is an $R$-valued point of $K$, the following computation shows that $f(x, g)$ is a point of $K^{\prime}$ :

$$
\begin{aligned}
\exp _{G^{\prime}}(\lambda \operatorname{Lie}(\alpha)(x)+\lambda \varphi(g)) & =\exp _{G^{\prime}}(\lambda \operatorname{Lie}(\alpha)(x)) \exp _{G^{\prime}}(\lambda \varphi(g)) \\
& =\exp _{G^{\prime}}(\lambda \operatorname{Lie}(\alpha)(x)) \text { by Lemma A.2.3 } \\
& =\exp _{G^{\prime}}(\operatorname{Lie}(\alpha)(\lambda x)) \\
& =\alpha\left(\exp _{G}(\lambda x)\right) \text { by functoriality of exp } \\
& =\alpha(g) \text { because } g=\exp _{G}(\lambda x)
\end{aligned}
$$

When $f$ is an isomorphism, applying the statement to $f^{-1}$, we find $\left(h^{*} f\right)(K)=K^{\prime}$. 


\subsection{Weil extension functor}

Now let $G$ be an affine and differentially flat $k$-group scheme. Thus $G$ as well as the adjoint representation $\operatorname{Lie}(G, I)$ are $k$-flat. We consider an arbitrary extension:

$$
1 \longrightarrow \operatorname{Lie}(G, I) \stackrel{i}{\longrightarrow} E \stackrel{\pi}{\longrightarrow} G \longrightarrow 1 .
$$

Then $E \rightarrow G$ is an fpqc torsor under $\operatorname{Lie}(G, I)$. It has a cohomology class in $\mathrm{H}^{1}(G$, $\operatorname{Lie}(G, I))$ which vanishes, being quasi-coherent cohomology of an affine scheme. It follows that $\pi$ has a section $s: G \rightarrow E$, and the extension becomes an $H$-extension. We may and do replace $s$ by $s(1)^{-1} \cdot s$ in order to ensure that $s(1)=1$. From $s$ we build a normalized cocycle $c: G \times G \longrightarrow \operatorname{Lie}(G, I)$ as follows:

$$
i\left(c\left(g, g^{\prime}\right)\right):=s(g) s\left(g^{\prime}\right) s\left(g g^{\prime}\right)^{-1} .
$$

These data give rise to the group $E_{c}$ as defined in Subsection 4.2.

4.3.1. Proposition. Let $1 \rightarrow \operatorname{Lie}(G, I) \rightarrow E \rightarrow G \rightarrow 1$ be an object of the category $\operatorname{Ext}(I) / k$. Let $s: G \rightarrow E$, with $s(1)=1$, be as chosen above, and $c$ the normalized cocycle derived from it. Let $\lambda \in k$.

(1) The map $\tau_{s}: E_{c} \rightarrow E,(x, g) \mapsto i(x) s(g)$ is an isomorphism of extensions.

(2) The closed normal subgroup scheme $K_{\lambda}(E):=\left(h^{*} \tau_{s}\right)\left(K_{\lambda}\left(E_{c}\right)\right) \subset h^{*} E$ does not depend on the choice of $s$.

(3) For all morphisms $f: E \rightarrow E^{\prime}$ in $\operatorname{Ext}(I) / k$ we have $\left(h^{*} f\right)\left(K_{\lambda}(E)\right) \subset K_{\lambda}\left(E^{\prime}\right)$, with equality if $f$ is an isomorphism.

If the extension $E$ is clear from context, we write $K_{\lambda}$ instead of $K_{\lambda}(E)$. Note that if $E$ is the trivial extension and $s=\alpha$, the map $\tau_{\alpha}$ is the map $\rho_{G}$ defined in paragraph A.1.

Proof. (1) follows from the constructions of $c$ and $E_{c}$.

For the proof of (2) and (3) we will rely on the following basic remark. Let $f: E \rightarrow E^{\prime}$ be a morphism in $\operatorname{Ext}(I) / k$. Let $\tau_{s}: E_{c} \rightarrow E$ and $\tau_{s^{\prime}}: E_{c^{\prime}} \rightarrow E^{\prime}$ be the isomorphisms associated to choices of sections $s, s^{\prime}$ preserving 1 and corresponding normalized cocycles $c, c^{\prime}$. Let $K_{\lambda, s}(E):=\left(h^{*} \tau_{s}\right)\left(K_{\lambda}\left(E_{c}\right)\right) \subset h^{*} E$ and similarly $K_{\lambda, s^{\prime}}\left(E^{\prime}\right):=\left(h^{*} \tau_{s^{\prime}}\right)\left(K_{\lambda}\left(E_{c^{\prime}}\right)\right) \subset h^{*} E^{\prime}$. We have a morphism of extensions:

$$
\rho=\tau_{s^{\prime}}^{-1} \circ f \circ \tau_{s}: E_{c} \longrightarrow E_{c^{\prime}} .
$$

According to Proposition 4.2.1(2) we have $\left(h^{*} \rho\right)\left(K_{\lambda}\left(E_{c}\right)\right) \subset K_{\lambda}\left(E_{c^{\prime}}\right)$. It follows that:

$$
\left(h^{*} f\right)\left(K_{\lambda, s}(E)\right)=\left(h^{*} f\right)\left(\left(h^{*} \tau_{s}\right)\left(K_{\lambda}\left(E_{c}\right)\right)\right)=\left(h^{*} \tau_{s^{\prime}}\right)\left(\left(h^{*} \rho\right)\left(K_{\lambda}\left(E_{c}\right)\right)\right) \subset\left(h^{*} \tau_{s^{\prime}}\right)\left(K_{\lambda}\left(E_{c^{\prime}}\right)\right)=K_{\lambda, s^{\prime}}\left(E^{\prime}\right) .
$$

When $f$ is an isomorphism, applying the statement to $f^{-1}$ gives equality.

(2) Applying the basic remark to $E=E^{\prime}$ and $f=\mathrm{id}: E \rightarrow E$ proves that $K_{\lambda, s}(E)=K_{\lambda, s^{\prime}}(E)$, that is, the subgroup $K_{\lambda, s}(E)$ does not depend on the choice of $s$. Since $\tau_{s}$ is an isomorphism of groups, the fact that $K_{\lambda}(E)$ is a closed normal subgroup scheme follows from Proposition 4.2.1(1).

(3) Applying the basic remark to a general $f$ gives the statement.

For an extension $1 \rightarrow \operatorname{Lie}(G) \rightarrow E \rightarrow G \rightarrow 1$, we let $K_{\lambda}:=K_{\lambda}(E)$ be the normal subgroup defined in the proposition. Point (4) in Proposition 3.2.1 gives motivation to consider $K_{-1}$. The fpqc quotient sheaf $h^{+} E:=h^{*} E / K_{-1}$ is representable by an affine flat $k[I]$-scheme (see Perrin [Per76, Cor. 0.2]).

4.3.2. Definition. We call Weil extension the quotient $h^{+} E:=h^{*} E / K_{-1}$. 


\subsubsection{Lemma. Weil extension is a functor $\operatorname{Ext}(I) / k \rightarrow \mathrm{Gr} / k[I]$.}

Proof. The $k[I]$-group scheme $G:=h^{*} E / K_{-1}$ is affine and flat. Let $s: G \rightarrow E$ be a section of $E \rightarrow G$ such that $s(1)=1$. By pullback, this induces a morphism $h^{*} G \rightarrow h^{*} E \rightarrow G$ which is the identity on the special fibre, hence an isomorphism, hence a rigidification. This proves that the functor of the statement is well-defined on objets. Proposition 4.3.1 proves that the functor is well-defined on morphisms.

\section{The equivalence of categories}

This section is devoted to the proof of Theorem A, which we recall below for ease of reading. The plan is as follows. In Subsection 5.1 we prove a preliminary result used in the proof of (1). In Subsection 5.2 we prove (1), (2), (4). Finally in Subsection 5.3 we prove (3).

5.0.1. Theorem. (1) The Weil restriction/extension functors provide quasi-inverse equivalences:

$$
\mathrm{Gr} / k[I] \underset{h^{+}}{\stackrel{h_{*}}{\rightleftarrows}} \operatorname{Ext}(I) / k \text {. }
$$

These equivalences commute with base changes $\operatorname{Spec}\left(k^{\prime}\right) \rightarrow \operatorname{Spec}(k)$.

(2) If $1 \rightarrow \mathscr{G}^{\prime} \rightarrow \mathscr{G} \rightarrow \mathscr{G}^{\prime \prime} \rightarrow 1$ is an exact sequence in $\mathrm{Gr} / k[I]$, then $1 \rightarrow h_{*} \mathscr{G}^{\prime} \rightarrow h_{*} \mathscr{G}_{\rightarrow} h_{*} \mathscr{G}^{\prime \prime}$ is exact in $\operatorname{Ext}(I) / k$. If moreover $\mathscr{G}^{\prime}$ is smooth then $1 \rightarrow h_{*} \mathscr{G}^{\prime} \rightarrow h_{*} \mathscr{G} \rightarrow h_{*} \mathscr{G}^{\prime \prime} \rightarrow 1$ is exact. In particular, $h_{*}$ is an exact equivalence between the subcategories of smooth objects endowed with their natural exact structure.

(3) The equivalence $h_{*}$ is a morphism of $\mathbb{O}_{k}$-module stacks fibred over $\mathrm{Gr} / k$, i.e. it transforms the addition and scalar multiplication of deformations of a fixed $G \in \mathrm{Gr} / k$ into the Baer sum and scalar multiplication of extensions of $G$ by $\operatorname{Lie}(G, I)$.

(4) Let $P$ be one of the properties: of finite type, smooth, connected, unipotent, split unipotent, solvable, commutative. Then $\mathscr{G} \in \mathrm{Gr} / k[I]$ has the property $P$ if and only if the $k$-group scheme $E=h_{*} \mathscr{G}$ has $P$.

\subsection{Equivariance of rigidifications under Lie algebra translation}

Let $\mathscr{G}$ be an affine, differentially flat, rigid $k[I]$-group scheme. Let $\sigma: h^{*} \mathscr{G}_{k} \stackrel{\sim}{\rightarrow} \mathscr{G}$ be a rigidification such that $\sigma(1)=1$. We consider the morphism of $k$-schemes:

$$
h_{*} \sigma: h_{*} h^{*} \mathscr{G}_{k} \longrightarrow h_{*} \mathscr{G} .
$$

This is not a morphism of group schemes, because source and target are not isomorphic groups in general. However, it satisfies an important equivariance property. To state it, note that source and target are extensions of $\mathscr{G}_{k}$ by $\operatorname{Lie}\left(\mathscr{G}_{k}, I\right)$; in particular both carry an action of $\operatorname{Lie}\left(\mathscr{G}_{k}, I\right)$ by left translation.

5.1.1. Proposition. With notation as above, the morphism of $k$-schemes

$$
h_{*} \sigma: h_{*} h^{*} \mathscr{G}_{k} \longrightarrow h_{*} \mathscr{G}
$$

is $\operatorname{Lie}\left(\mathscr{G}_{k}, I\right)$-equivariant.

Proof. We write simply $\mathbb{O}$ instead of $\mathbb{O}_{k}$ or $\mathbb{O}_{k[I]}$ when the base is clear from context. Consider the extension of $\sigma$ to the group algebras:

$$
\sigma^{\prime}:=h_{*} \mathbb{O}[\sigma]: h_{*} \mathbb{O}\left[h^{*} \mathscr{G}_{k}\right] \rightarrow h_{*} \mathbb{O}[\mathscr{G}]
$$


Note that by compatibility of $\mathbb{O}[-]$ with base change and Weil restriction (see Proposition 2.3.1, (2)-(4)), we have $h_{*} \mathbb{O}\left[h^{*} \mathscr{G}_{k}\right] \stackrel{\sim}{\rightarrow} h_{*} h^{*} \mathbb{O}\left[\mathscr{G}_{k}\right] \stackrel{\sim}{\rightarrow} \mathbb{O}\left[\mathscr{G}_{k}\right][I]$. We obtain a commutative diagram:

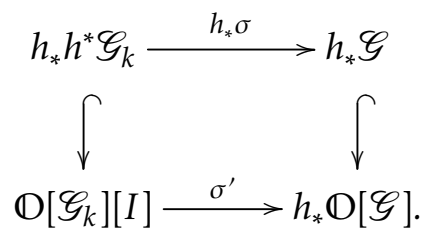

We identify $I \cdot \mathbb{O}\left[\mathscr{G}_{k}\right]:=\mathbb{O}\left[\mathscr{G}_{k}\right] \otimes_{\mathbb{O}_{k}} \mathbb{V}\left(I^{\vee}\right)$ as the ideal generated by $I$ inside both algebras $\mathbb{O}\left[\mathscr{G}_{k}\right][I]$ and $h_{*} \mathbb{O}[\mathscr{G}]$, see Corollary 3.3.5. With this convention we formulate:

Claim. We have $\sigma^{\prime}(y+x)=\sigma^{\prime}(y)+x$ for all points $y \in \mathbb{O}\left[\mathscr{G}_{k}\right][I]$ and $x \in I \cdot \mathbb{O}\left[\mathscr{G}_{k}\right]$.

To prove this, we introduce another copy $J=I$ of our square-zero ideal as follows:

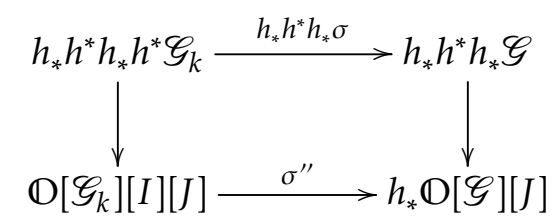

where we have set $\sigma^{\prime \prime}=h_{*} h^{*} \sigma^{\prime}$ for brevity. Let $s, t: \mathbb{O}\left[\mathscr{G}_{k}\right][I] \times J \cdot \mathbb{O}\left[\mathscr{G}_{k}\right][I][J] \rightarrow h_{*} \mathbb{O}[\mathscr{G}][J]$ be defined by

$$
s(y, x)=\sigma^{\prime \prime}(y+x) \quad \text { and } \quad t(y, x)=\sigma^{\prime \prime}(y)+x .
$$

Then $s$ and $t$ are equal modulo $I$ because of the fact that $\sigma$ is the identity modulo $I$. Clearly they are also equal modulo $J$. Since $h_{*} \mathbb{O}[\mathscr{G}][J]$ is flat as an $\mathbb{O}_{k}[I][J]$-module, we deduce that $s-t$ takes its values in the ideal $I J \cdot h_{*} \mathbb{O}[\mathscr{G}][I]$. Setting $J=I$, that is composing with the morphism $h_{*} \mathbb{O}[\mathscr{G}][J] \rightarrow h_{*} \mathbb{O}[\mathscr{G}]$ that takes a section of $J$ to the corresponding section of $I$, we obtain the claim.

We now conclude the proof of the proposition. We write $\star_{1}$ and $\star_{2}$ the multiplications of $h_{*} h^{*} \mathscr{G}_{k}$ and $h_{*} \mathscr{G}$ respectively, extended to $h_{*} \mathbb{O}\left[h^{*} \mathscr{G}_{k}\right]$ and $h_{*} \mathbb{O}[\mathscr{G}]$. It is enough to show more generally that

$$
h_{*} \sigma: h_{*} \mathbb{O}\left[h^{*} \mathscr{G}_{k}\right] \longrightarrow h_{*} \mathbb{O}[\mathscr{G}]
$$

is $\left(1+I \cdot \mathbb{O}\left[\mathscr{G}_{k}\right]\right)$-equivariant. We take points $x \in I \cdot \mathbb{O}\left[\mathscr{G}_{k}\right]$ and $y \in h_{*} \mathbb{O}\left[h^{*} \mathscr{G}_{k}\right]$ and compute:

$$
\begin{aligned}
\left(h_{*} \sigma\right)\left((1+x) \star_{1} y\right) & =\left(h_{*} \sigma\right)\left(y+x \star_{1} y\right) \\
& =\left(h_{*} \sigma\right)(y)+x \star_{1} y \text { by the Claim above, } \\
& =\left(h_{*} \sigma\right)(y)+x \star_{2} y \text { by Corollary } 3.3 .5 \text { since } \star_{1}=\star_{2} \text { modulo } I, \\
& =\left(h_{*} \sigma\right)(y)+x \star_{2}\left(h_{*} \sigma\right)(y) \text { by Corollary } 3.3 .5 \text { since } \sigma=\text { id modulo } I, \\
& =(1+x) \star_{2}\left(h_{*} \sigma\right)(y) .
\end{aligned}
$$

This proves that $h_{*} \sigma$ is $\operatorname{Lie}\left(\mathscr{G}_{k}, I\right)$-equivariant.

\subsection{Proof of the main theorem: equivalence and exactness}

5.2.1. Proof of 5.0.1(1). We shall prove that the functors

$$
\mathrm{Gr} / k[I] \underset{h^{+}}{\stackrel{h_{*}}{\rightleftarrows}} \operatorname{Ext}(I) / k
$$


provide quasi-inverse equivalences that commute with base changes $\operatorname{Spec}\left(k^{\prime}\right) \rightarrow \operatorname{Spec}(k)$. Firstly, we prove that $h^{+} \circ h_{*}$ is isomorphic to the identity. Let $\mathscr{G} \rightarrow \operatorname{Spec}(k[I])$ be an affine, differentially flat, rigid $k[I]$-group scheme. Let $E=h_{*} \mathscr{G}$ be the associated extension:

$$
1 \longrightarrow \operatorname{Lie}\left(\mathscr{G}_{k}, I\right) \longrightarrow E \longrightarrow \mathscr{G}_{k} \longrightarrow 1
$$

We fix a rigidification $\sigma: h^{*} \mathscr{G}_{k} \stackrel{\sim}{\rightarrow} \mathscr{G}$ such that $\sigma(1)=1$. We know from Proposition 5.1.1 that the map $h_{*} \sigma: h_{*} h^{*} \mathscr{G}_{k} \longrightarrow h_{*} \mathscr{G}$ is $\operatorname{Lie}\left(\mathscr{G}_{k}, I\right)$-equivariant. If we use the letter $\gamma$ to denote the inclusions of $\operatorname{Lie}\left(\mathscr{G}_{k}, I\right)$ into the relevant extensions, this can be written:

$$
\left(h_{*} \sigma\right)\left(\gamma_{h_{*}} h^{*} \mathscr{G}_{k}(x) \cdot y\right)=\gamma_{h_{*}} \mathscr{G}(x) \cdot\left(h_{*} \sigma\right)(y), \quad \text { all } x \in \operatorname{Lie}\left(\mathscr{C}_{k}, I\right), y \in h_{*} h^{*} \mathscr{G}_{k} .
$$

Restricting to $y$ in the image of $\alpha=\alpha \mathscr{G}_{k}: \mathscr{G}_{k} \hookrightarrow h_{*} h^{*} \mathscr{G}_{k}$, so $\tau_{\alpha}=\rho \mathscr{G}_{k}$, we obtain:

$$
h_{*} \sigma \circ \tau_{\alpha}=\tau_{s} .
$$

Using functoriality of $\beta$ and the fact that $\sigma(1)=1$, we build a commutative diagram:

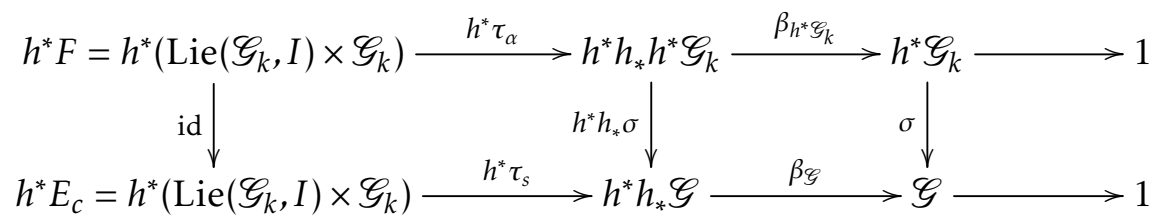

Here the horizontal maps are morphisms of groups and the vertical maps are not morphisms of groups (not even the leftmost map id $: h^{*} F \rightarrow h^{*} E_{c}$ ). Note also that $F$ is $E_{0}=\mathrm{T}\left(\mathscr{G}_{k}, I\right)$, that is, the extension $E_{c}$ with the zero cocycle $c=0$. Now we consider $K_{-1}(E)$ as defined in Proposition 4.3.1. According to Proposition 3.2.1(4), the group $K_{-1}\left(E_{0}\right) \subset E_{0}$ is the kernel of $\beta_{h^{*} \mathscr{G}_{k}} \circ h^{*} \tau_{\alpha}$. On one hand the identity takes $K_{-1}\left(E_{0}\right)$ to $K_{-1}\left(E_{c}\right)$, and on the other hand the map $h_{*} \sigma$ takes $\operatorname{ker}\left(\beta_{h^{*}} \mathscr{G}_{k}\right)$ onto $\operatorname{ker}(\beta \mathscr{G})$ since it takes 1 to 1 . By commutativity of the left-hand square, we find $K_{-1}(E)=\operatorname{ker}\left(\beta_{\mathscr{G}}\right)$ and thefore $\beta_{\mathscr{G}}$ induces an isomorphism $h^{+} E=h^{*} E / K_{-1}(E) \simeq \mathscr{G}$ which is visibly functorial.

Secondly, we prove that $h_{*} \circ h^{+}$is isomorphic to the identity. Let $1 \rightarrow \operatorname{Lie}(G, I) \rightarrow E \rightarrow G \rightarrow 1$ be an extension. We fix a section $s: G \rightarrow E$ such that $s(1)=1$ and we let $c$ be the normalized cocycle defined by $s$. Let $K_{-1}=K_{-1}(E) \subset h^{*} E$ be the closed normal subgroup defined in Proposition 4.3.1, and let $\mathscr{G}:=h^{+} E=h^{*} E / K_{-1}$ with quotient map $\pi: h^{*} E \rightarrow \mathscr{G}$. Define $\sigma=\pi \circ h^{*} s: h^{*} G \rightarrow \mathscr{G}$. Since $i^{*} K_{-1}=\operatorname{Lie}(G, I)$ as a subgroup of $E$, we see that $i^{*} \mathscr{G} \simeq G$ and $i^{*} \sigma$ is the identity of $G$. Since $G$ is $k$-flat, it follows that $\sigma$ is an isomorphism. From the construction of $K_{-1}$, we see that after we compose with the isomorphisms

$$
h^{*} \tau_{s}: h^{*} h_{*} h^{*} G \stackrel{\sim}{\longrightarrow} h^{*} E \text { and } \sigma: h^{*} G \stackrel{\sim}{\rightarrow} \mathscr{G},
$$

the flat surjection $\pi: h^{*} E \rightarrow \mathscr{G}$ is identified with the counit of the adjunction:

$$
\beta_{h^{*} G}: h^{*} h_{*} h^{*} G \longrightarrow h^{*} G
$$

We apply $h_{*}$ and obtain the commutative diagram:

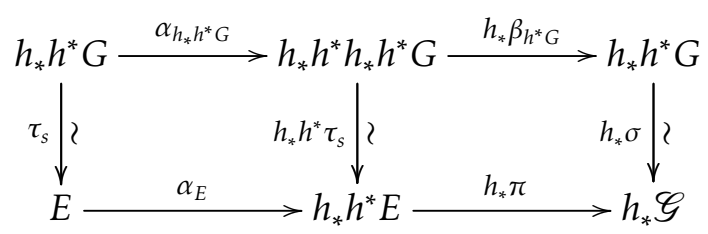

Since the top row is the identity, we see that the bottom row is an isomorphism, i.e. $E \stackrel{\sim}{\rightarrow} h_{*} \mathscr{G}$. Again, it is clear that this isomorphism is functorial.

Finally, we consider the commutation with base changes. For Weil restriction, this is a standard fact. For Weil extension, this follows from base change commutation for pullbacks and for quotients by flat subgroups. 
5.2.2. Proof of 5.0.1(2). Let $1 \rightarrow \mathscr{G}^{\prime} \rightarrow \mathscr{G} \rightarrow \mathscr{G}^{\prime \prime} \rightarrow 1$ be an exact sequence in $\mathrm{Gr} / k[I]$. Then the exact sequences with solid arrows are exact:

$$
\begin{gathered}
1 \longrightarrow \mathscr{G}_{k}^{\prime} \longrightarrow \mathscr{G}_{k} \longrightarrow \mathscr{G}_{k}^{\prime \prime} \longrightarrow 1 \\
1 \longrightarrow \operatorname{Lie}\left(\mathscr{G}_{k}^{\prime}, I\right) \longrightarrow \operatorname{Lie}\left(\mathscr{G}_{k}, I\right) \longrightarrow \operatorname{Lie}\left(\mathscr{G}_{k}^{\prime \prime}, I\right) \cdots \cdots 1
\end{gathered}
$$

Moreover, if $\mathscr{G}^{\prime}$ is smooth then the second sequence is exact also if we include the dotted arrow. By an easy diagram chase, we find that the commutative diagram below has exact rows (again including dotted arrows if $\mathscr{G}^{\prime}$ is smooth):

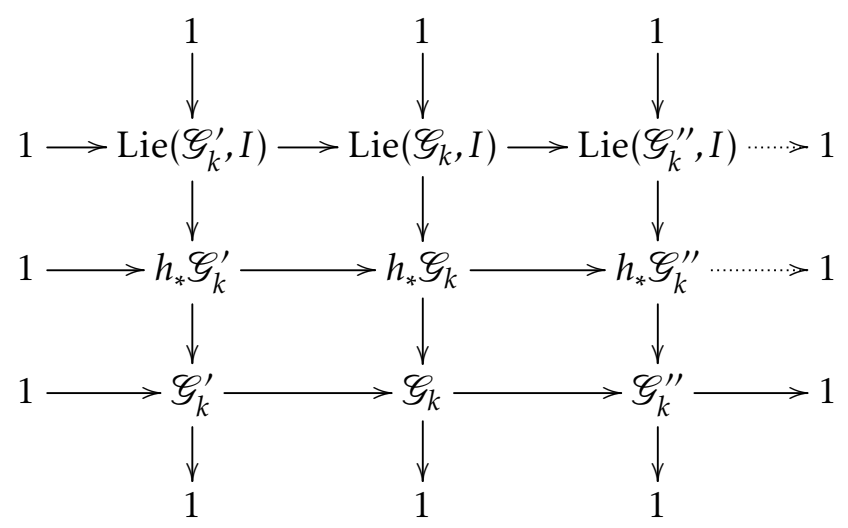

This proves the claim.

5.2.3. Proof of 5.0.1(4). If $\mathscr{G}$ is of finite type, or smooth, or connected, or unipotent, or split unipotent, or solvable, then $G=i * \mathscr{G}$ as well as $\operatorname{Lie}(G, I)$ have the same property. It follows that $E=h_{*} \mathscr{G}$ has the property. Moreover, if $\mathscr{G}$ is commutative then $E$ also.

Conversely, if $E$ is of finite type, or smooth, or connected, or unipotent, or split unipotent, or solvable, or commutative, then $h^{*} E$ has the same property. Therefore the quotient $h^{+} E:=h^{*} E / K_{-1}$ has the same property.

\subsection{Proof of the main theorem: isomorphism of $\mathbb{O}_{k}$-module stacks}

In this paragraph, we prove 5.0.1(3), i.e. that the Weil restriction functor $h_{*}: \mathrm{Gr} / k[I] \rightarrow \operatorname{Ext}(I) / k$ exchanges the addition and the scalar multiplication on both sides. Before we start, we point out that these properties will imply that the image of a trivial deformation group scheme $\mathscr{G}=h^{*} G$ under Weil restriction is the tangent bundle (i.e. trivial) extension $\mathrm{T}(G, I)$, a fact which can be shown directly using Proposition 3.2.1(4).

We work in the fibre category over a fixed $G \in \mathrm{Gr} / k$ and we set $L:=\operatorname{Lie}(G, I)$. Let $\mathscr{G}_{1}, \mathscr{G}_{2} \in \mathrm{Gr} / k[I]$ with identifications $i^{*} \mathscr{G}_{1} \simeq G \simeq i^{*} \mathscr{G}_{2}$. For clarity, we introduce three copies $I_{1}=I_{2}=I$ of the same finite free $k$-module. For $c=1,2$ we have obvious maps:

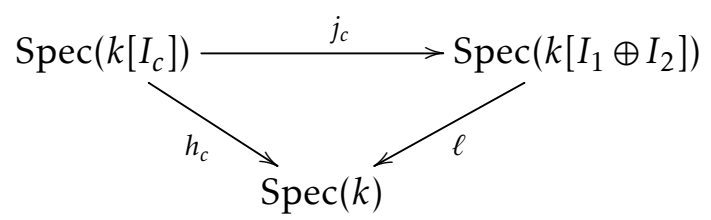

Set $\mathscr{G}^{\prime}=\mathscr{G}_{1} \sqcup_{G} \mathscr{G}_{2} \in \mathrm{Gr} / k\left[I_{1} \oplus I_{2}\right]$, so $\mathscr{G}_{1}+\mathscr{G}_{2}=j^{*} \mathscr{G}^{\prime}$ where $j: \operatorname{Spec}(k[I]) \hookrightarrow \operatorname{Spec}(k[I \oplus I])$ is the closed immersion induced by the addition morphism $I \oplus I \rightarrow I$. We have a morphism

$$
\xi: \ell_{*} \mathscr{G}^{\prime} \longrightarrow h_{1, *} \mathscr{G}_{1} \times_{G} h_{2, *} \mathscr{G}_{2}
$$


whose component $\xi_{c}: \ell_{*} \mathscr{G}^{\prime} \rightarrow h_{c, *} \mathscr{G}_{c}$ is the $\ell_{*}$-pushforward of the adjunction $\mathscr{G}^{\prime} \rightarrow j_{c, *} j_{c}^{*} \mathscr{G}^{\prime}=j_{c, *} \mathscr{G}_{c}$. Besides, we have a morphism

$$
\omega: \ell_{*} \mathscr{G}^{\prime} \longrightarrow h_{*}\left(\mathscr{G}_{1}+\mathscr{G}_{2}\right)
$$

obtained as the $\ell_{*}$-pushforward of the adjunction $\mathscr{G}^{\prime} \rightarrow j_{*} j^{*} \mathscr{G}^{\prime}=j_{*}\left(\mathscr{G}_{1}+\mathscr{G}_{2}\right)$.

5.3.1. Lemma. The morphism $\xi$ is an isomorphism and it induces an isomorphism of extensions on the bottom row of the following commutative square:

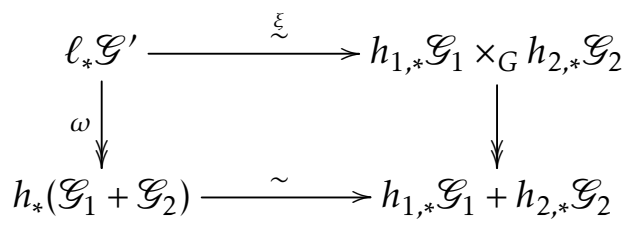

Proof. Write $G=\operatorname{Spec}(A)$ and $\mathscr{G}_{c}=\operatorname{Spec}\left(\mathscr{A}_{c}\right)$ so $\mathscr{G}^{\prime}=\operatorname{Spec}\left(\mathscr{A}_{1} \times_{A} \mathscr{A}_{2}\right)$. There is a morphism of algebra schemes $\xi^{\prime}: \ell_{*} \mathbb{O}\left[\mathscr{G}^{\prime}\right] \rightarrow h_{1, *} \mathbb{O}\left[\mathscr{G}_{1}\right] \times_{\mathbb{O}_{k}[G]} h_{2, *} \mathbb{O}\left[\mathscr{G}_{2}\right]$ contructed in the same way as $\xi$. It order to describe $\xi^{\prime}$ we can express the Weil restrictions in terms of $I$-compatible maps as in Lemma 3.1.2. For a $k$-algebra $R$, we have:

$$
\begin{aligned}
\operatorname{Homc}_{k}\left(\mathscr{A}_{1} \times_{A} \mathscr{A}_{2}, I_{1} R \oplus I_{2} R\right) & \stackrel{\xi^{\prime}(R)}{\longrightarrow} \operatorname{Homc}_{k}\left(\mathscr{A}_{1}, I_{1} R\right) \underset{\operatorname{Hom}_{k}(A, R)}{\times} \operatorname{Homc}_{k}\left(\mathscr{A}_{2}, I_{2} R\right) \\
v & \longmapsto\left(v_{1}, v_{2}\right)
\end{aligned}
$$

where $v_{1}$ (resp. $v_{2}$ ) is $v$ modulo $I_{2}$ (resp. $I_{1}$ ). This is a bijection whose inverse sends a pair $\left(v_{1}, v_{2}\right)$ with $v_{1}^{*}=v_{2}^{*}: A \rightarrow R$ to the map $v: \mathscr{A}_{1} \times_{A} \mathscr{A}_{2} \rightarrow I_{1} R \oplus I_{2} R,\left(a_{1}, a_{2}\right) \mapsto v_{1}\left(a_{1}\right)+v_{2}\left(a_{2}\right)$. The morphism $\xi$ is the bijection obtained by restriction of $\xi^{\prime}$ to the subsets of algebra maps as in Lemma 3.1.2(2). Namely, an algebra map is of the form $f=\bar{v}+v$ where $v$ is $I$-compatible, and $\omega(R)$ sends $f$ to $\left(f_{1}, f_{2}\right)$ while $\omega(R)^{-1}$ sends $\left(f_{1}=\bar{v}+v_{1}, f_{2}=\bar{v}+v_{2}\right)$ to $f=\bar{v}+v_{1}+v_{2}$.

In order to describe $\omega$ note that $\left(h_{*}\left(\mathscr{G}_{1}+\mathscr{G}_{2}\right)\right)(R)=\operatorname{Hom}_{k\left[I_{1} \oplus I_{2}\right]-\operatorname{Alg}}\left(\mathscr{A}_{1} \times_{A} \mathscr{A}_{2}, R[I]\right)$ where $R[I]$ is a $k\left[I_{1} \oplus I_{2}\right]$-algebra via the map $k\left[I_{1} \oplus I_{2}\right] \rightarrow k[I]$ induced by addition $+: I \oplus I \rightarrow I$. Then $\omega(R)$ sends $f$ to the composition

$$
\mathscr{A}_{1} \times_{A} \mathscr{A}_{2} \stackrel{f}{\longrightarrow} R\left[I_{1} \oplus I_{2}\right] \stackrel{+}{\longrightarrow} R[I] .
$$

Thus $\omega(R)$ is surjective because $R\left[I_{1} \oplus I_{2}\right] \longrightarrow R[I]$ has $R$-algebra sections, i.e. $\omega$ is a surjection of functors. Its kernel is the set of maps $f=\bar{v}+v_{1}+v_{2}$ such that $v_{1}+v_{2}=d_{1}+d_{2}: \mathscr{A}_{1} \times_{A} \mathscr{A}_{2} \rightarrow R[I]$, with $e_{c}=d_{c}^{*}+d_{c}: \mathscr{A}_{c} \rightarrow k\left[I_{c}\right]$ the counits of the Hopf algebras. After translation by the derivations as indicated by Proposition 3.2.1(3), on the side of extensions the kernel is $\operatorname{ker}(+: L \times L \rightarrow L)$, giving rise to a quotient isomorphic to the Baer sum extension $h_{1, *} \mathscr{G}_{1}+h_{2, *} \mathscr{G}_{2}$.

It remains to prove that $h_{*}: \mathrm{Gr} / k[I] \rightarrow \operatorname{Ext}(I) / k$ exchanges the scalar multiplication on both sides. Let $\mathscr{G} \in \mathrm{Gr} / k[I]$ with an identification $i^{*} \mathscr{G} \simeq G$. We will reduce to a situation similar as that of Lemma 5.3.1 thanks to the following trick.

5.3.2. Lemma. Let $j_{\lambda}: \operatorname{Spec}(k[I]) \hookrightarrow \operatorname{Spec}\left(k\left[I_{1} \oplus I_{2}\right]\right)$ be the closed immersion defined by the surjective $k$-algebra map $I_{1} \oplus I_{2} \rightarrow I, i_{1} \oplus i_{2} \mapsto \lambda i_{1}+i_{2}$. Then we have $s_{\lambda}^{*} \mathscr{G} \stackrel{\sim}{\rightarrow} j_{\lambda}^{*}\left(\mathscr{G} \amalg_{G} h^{*} G\right)$ canonically.

Proof. If we think of Speck $\left[I_{1} \oplus I_{2}\right]$ as the coproduct Spec $k\left[I_{1}\right] \amalg_{\text {Speck }} \operatorname{Spec} k\left[I_{2}\right]$, the map $j_{\lambda}$ is the composition $\left(s_{\lambda} \amalg \mathrm{id}\right) \circ j$ as follows:

$$
\operatorname{Spec}(k[I]) \stackrel{j}{\longrightarrow} \operatorname{Spec}\left(k\left[I_{1} \oplus I_{2}\right]\right) \stackrel{s_{\lambda} \text { Lid }}{\longrightarrow} \operatorname{Spec}\left(k\left[I_{1} \oplus I_{2}\right]\right) .
$$

It follows that:

$$
j_{\lambda}^{*}\left(\mathscr{G} \amalg_{G} h^{*} G\right)=j^{*}\left(\left(s_{\lambda} \amalg \mathrm{id}\right)^{*}\left(\mathscr{G} \amalg_{G} h^{*} G\right)\right)=j^{*}\left(s_{\lambda}^{*} \mathscr{G} h^{*} G\right)=s_{\lambda}^{*} \mathscr{G}+h^{*} G=s_{\lambda}^{*} \mathscr{G},
$$

because $h^{*} G$ is the neutral element for the sum in the fibre category of $\mathrm{Gr} / k[I] \rightarrow \mathrm{Gr} / k$ at $G$. 
Set $\mathscr{G}_{1}=\mathscr{G}$ and $\mathscr{G}_{2}=h^{*} G$. Recall that $L=\operatorname{Lie}(G, I)$. The Weil restrictions are $E:=E_{1}=h_{*} \mathscr{G}$ and the trivial extension $E_{2}=h_{*} h^{*} G=L \rtimes G$. As in Lemma 5.3.1, there are morphisms $\xi: \ell_{*} \mathscr{G}^{\prime} \rightarrow E_{1} \times_{G} E_{2}=E \times L$.

5.3.3. Lemma. The morphism $\xi$ is an isomorphism which induces an isomorphism of extensions on the bottom row of the following commutative square:

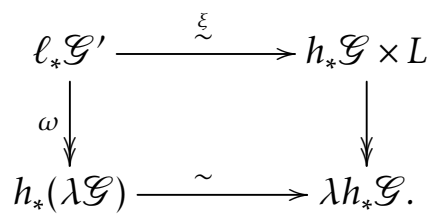

Proof. The proof is the same as that of Lemma 5.3.1 except that in the final step we use the map $I \oplus I \rightarrow I$, $i_{1} \oplus i_{2} \mapsto \lambda i_{1}+i_{2}$. Again this morphism is surjective and on the side of extensions, the kernel corresponds to the kernel of $L \times L \rightarrow L,\left(v_{1}, v_{2}\right) \mapsto \lambda v_{1}+v_{2}$. The quotient of $E \times L$ by this kernel is exactly the extension $\lambda E$, the pushout of the diagram:

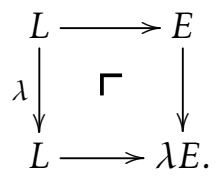

This finishes the proof.

\section{Dieudonné theory for unipotent groups over the dual numbers}

In this section, as an application of Theorem A, we give a classification of smooth, unipotent group schemes over the dual numbers of a perfect field $k$, in terms of extensions of Dieudonné modules. So throughout the section, the ring $k$ is a perfect field of characteristic $p>0$.

\subsection{Reminder on Dieudonné theory}

We denote by $W$ the Witt ring scheme over $k$ and F,V its Frobenius and Verschiebung endomorphisms. For all $n \geqslant 1$, we write $W_{n}:=W / \mathrm{V}^{n} W$ the ring scheme of Witt vectors of length $n$. We use the same notation also for these operators over the $R$-points, with $R$ a $k$-algebra. We also define $\widetilde{V}: W_{n} \longrightarrow W_{n+1}$ as the morphism induced on $W_{n}$ by the composition

$$
W \stackrel{\mathrm{V}}{\longrightarrow} W \stackrel{\pi_{n+1}}{\longrightarrow} W_{n+1}
$$

where $\pi_{n+1}$ is the natural projection.

The Dieudonné ring $\mathbb{D}$ is the $W(k)$-algebra generated by two variables $\mathbb{F}$ and $\mathbb{V}$ with the relations:

$$
\begin{aligned}
\mathbb{F} x & =\mathrm{F}(x) \mathbb{F} \\
x \mathbb{V} & =\mathbb{V} F(x) \\
\mathbb{F} \mathbb{V} & =\mathbb{V} \mathbb{F}=p,
\end{aligned}
$$

for varying $x \in W(k)$. A Dieudonné module is a left $\mathbb{D}$-module. A Dieudonné module $M$ is called erasable if for any $m \in M$ there exists a positive integer $n$ such that $\mathbb{V}^{n} m=0$.

Let $R$ be a $k$-algebra. Then, for any $n \geqslant 1$, we make $W_{n}(R)$ a left $\mathbb{D}$-module with the rules:

$$
\begin{aligned}
\mathbb{F} \cdot u & =\mathrm{F}(u) \\
\mathbb{V} \cdot u & =\mathrm{V}(u) \\
x \cdot u & =\mathrm{F}^{1-n}(x) u
\end{aligned}
$$


for all $u \in W_{n}(R)$ and $x \in W(k)$. The twist in the latter definition is designed to make $\widetilde{\mathrm{V}}: W_{n}(R) \rightarrow W_{n+1}(R)$ a morphism of $\mathbb{D}$-modules, see Demazure and Gabriel [DG70, chap. V, $\S 1$, no 3.3]. All of this is functorial in $R$ and gives $W_{n}$ a structure of $\mathbb{D}$-module scheme. In particular, $\operatorname{End}_{k}\left(W_{n}\right)$ is a $\mathbb{D}$-module. According to [DG70, chap. V, $\S 1$, no 3.4] the morphism $\mathbb{D} \rightarrow \operatorname{End}_{k}\left(W_{n}\right)$ induces an isomorphism of $\mathbb{D}$-modules:

$$
\mathbb{D} / \mathbb{D V}^{n} \stackrel{\sim}{\rightarrow} \operatorname{End}_{k}\left(W_{n}\right) .
$$

If $U$ is a commutative, unipotent $k$-group scheme, the set $\operatorname{Hom}_{k}\left(U, W_{n}\right)$ is a Dieudonné module with its structure given by postcomposition, i.e. for any $f: U \rightarrow W_{n}$ :

$$
\begin{aligned}
\mathbb{F} \cdot f & =\mathrm{F} \circ f \\
\mathbb{V} \cdot f & =\mathrm{V} \circ f \\
x \cdot f & =\mathrm{F}^{1-n}(x) f,
\end{aligned}
$$

all $x \in W(k)$. We define the Dieudonné module of $U$ as:

$$
\underline{M}(U):=\lim _{\vec{n}} \operatorname{Hom}_{k}\left(U, W_{n}\right)
$$

where the transition maps of the inductive system are induced by $\widetilde{V}: W_{n} \rightarrow W_{n+1}$. Since $\operatorname{Hom}_{k}\left(U, W_{n}\right)$ is killed by $\mathbb{V}^{n}$ and $\underline{M}(U)$ is a union of these subgroups, we see that $\underline{M}(U)$ is erasable. If $M$ is a Dieudonné module, we define its Frobenius twist $M^{(p)}$ as the module with underlying group $M^{(p)}=M$ and $\mathbb{D}$-module structure given by:

$$
\mathbb{F}_{M^{(p)}}=\mathbb{F}_{M}, \quad \mathbb{V}_{M^{(p)}}=\mathbb{V}_{M}, \quad x_{M^{(p)}}=\mathrm{F}^{-1}(x)_{M} \quad \text { for all } x \in W(k) .
$$

Then the maps $\mathbb{F}_{M}: M^{(p)} \rightarrow M$ and $\mathbb{V}_{M}: M \rightarrow M^{(p)}$ are visibly $\mathbb{D}$-linear. Moreover, let $\mathrm{CU} / k$ be the category of commutative unipotent $k$-group schemes; according to [DG70, chap. V, $\S 1,4.5]$ we have a canonical isomorphism $\underline{M}(U)^{(p)} \stackrel{\sim}{\rightarrow} \underline{M}\left(U^{(p)}\right)$ for all $U \in \mathrm{CU}$ with Frobenius twist $U^{(p)}$.

6.1.1. Theorem. Let $\mathbb{D}$-Mod be the category of Dieudonné modules. The contravariant functor

$$
\underline{M}: \mathrm{CU} / k \rightarrow \mathbb{D}-\mathrm{Mod}
$$

is exact, fully faithful with essential image the full subcategory of erasable Dieudonné modules. It transforms the Frobenius (resp. Verschiebung) of $U$ into the Frobenius (resp. Verschiebung) of $\underline{M}(U)$. Moreover a unipotent group scheme $U$ is of finite type if and only if $\underline{M}(U)$ is of finite type.

Proof. See [DG70, chap. V, § 1, 4.3, 4.4, 4.5].

\subsection{Dieudonné theory over the dual numbers}

Before stating our Dieudonné classification, we need to define the notions of Lie algebra and smoothness of Dieudonné modules. We let $\mathbb{D}$ - $\operatorname{Mod}^{e} \subset \mathbb{D}$-Mod be the subcategory of erasable $\mathbb{D}$-modules.

6.2.1. Definition. Let $M \in \mathbb{D}$-Mod be a Dieudonné module. We define the Lie algebra of $M$ by:

$$
\text { Lie } M:=(M / \mathbb{F} M) \otimes_{k} k[\mathbb{F}] .
$$

If $I$ a finite dimensional $k$-vector space, the $I$-Lie algebra of $M$ is $\operatorname{Lie}(M, I):=\operatorname{Lie} M \otimes_{k} I^{\vee}$. This gives rise to endofunctors $\operatorname{Lie}(-)$ and $\operatorname{Lie}(-, I)$ of the category $\mathbb{D}-\operatorname{Mod}^{e}$. 
6.2.2. Proposition. We have an isomorphism, functorial in $U \in \mathrm{CU} / k$ :

$$
\operatorname{Lie}(\underline{M}(U), I) \stackrel{\sim}{\longrightarrow} \underline{M}(\operatorname{Lie}(U, I)) .
$$

Proof. We start with the case of dimension one $I=k$, so $\operatorname{Lie}(U, k \varepsilon)=\operatorname{Lie}(U)$. Let $U^{\prime}$ be the kernel of Frobenius in $U$ and $M=\underline{M}(U), M^{\prime}=\underline{M}\left(U^{\prime}\right)$. We have an exact sequence:

$$
0 \longrightarrow U^{\prime} \longrightarrow U \stackrel{\mathrm{F}_{U}}{\longrightarrow} U^{(p)} .
$$

We deduce isomorphisms Lie $U \stackrel{\sim}{\rightarrow}$ Lie $U^{\prime}$ and $M^{\prime}=M / \mathbb{F} M$. In the sequel set $L_{U}:=(\operatorname{Lie} U)(k)$, a $k$-vector space. Since $U^{\prime}$ is a finite commutative group scheme, according to Fontaine [Fo77, chap. III, 4.2] there is a canonical isomorphism:

$$
\eta_{U^{\prime}}: M^{\prime} / \mathbb{F} M^{\prime} \stackrel{\sim}{\rightarrow} L_{U^{\prime}}^{\vee}
$$

We deduce a composed isomorphism $\eta_{U}$ as follows:

$$
M / \mathbb{F} M \stackrel{\sim}{\longrightarrow} M^{\prime} / \mathbb{F} M^{\prime} \stackrel{\eta_{U^{\prime}}}{\rightarrow} L_{U^{\prime}}^{\vee} \stackrel{\sim}{\rightarrow} L_{U}^{\vee} .
$$

From the isomorphism of $k$-group schemes Lie $U \simeq \mathbb{V}\left(L_{U}^{\vee}\right)$, we deduce

$$
M(\operatorname{Lie} U)=\operatorname{Hom}_{k}\left(\mathbb{V}\left(L_{U}^{\vee}\right), \mathbb{G}_{a}\right) \stackrel{\sim}{\rightarrow} L_{U}^{\vee} \otimes k[\mathbb{F}] .
$$

By tensoring $\eta_{U}$ with $k[\mathbb{F}]$, we find

$$
\text { Lie } M=(M / \mathbb{F} M) \otimes_{k} k[\mathbb{F}] \stackrel{\sim}{\longrightarrow} L_{U}^{\vee} \otimes k[\mathbb{F}] \stackrel{\sim}{\rightarrow} M(\text { Lie } U) .
$$

The result for general $I$ follows since $\operatorname{Lie}(U, I) \simeq \operatorname{Lie} U \otimes_{\mathbb{O}_{k}} \mathbb{V}\left(I^{\vee}\right)$ and $\operatorname{Lie}(M, I)=\operatorname{Lie} M \otimes_{k} I^{\vee}$.

We can characterize the functor Lie on Dieudonné modules by its values on the modules $\mathbb{D} / \mathbb{D} \mathbb{V}^{n}$.

6.2.3. Proposition. There exists a unique covariant functor $\mathscr{L}: \mathbb{D}-\operatorname{Mod}^{e} \rightarrow \mathbb{D}-\operatorname{Mod}^{e}$ with the following properties:

(1) $\mathscr{L}$ is right exact and commutes with filtering inductive limits;

(2) $\mathscr{L}\left(\mathbb{D} / \mathbb{D} \mathbb{V}^{n}\right)=k[\mathbb{F}]^{n}$ for all $n \geqslant 1$;

(3) $\mathscr{L}: \operatorname{End}\left(\mathbb{D} / \mathbb{D} \mathbb{V}^{n}\right) \rightarrow \operatorname{End}\left(k[\mathbb{F}]^{n}\right)$ sends

- IF to 0 ;

- $\mathbb{V}$ to the endomorphism $\left(a_{0}, a_{1}, \ldots, a_{n-1}\right) \mapsto\left(0, a_{0}, a_{1}, \ldots, a_{n-2}\right)$;

- and multiplication by $x=\left(x_{0}, x_{1}, x_{2}, \ldots\right) \in W(k)$ to the diagonal endomorphism with diagonal entries $\left(x_{0}, x_{0}^{p}, \ldots, x_{0}^{p^{n-1}}\right)$.

Proof. Uniqueness. The key is the fact that $\mathbb{D} / \mathbb{D} \mathbb{V}^{n}$ is a projective generator of the full subcategory $C_{n}:=\left(\mathbb{D} \text {-Mod }{ }^{e}\right)^{\mathbb{V}^{n}=0}$ of objects killed by $\mathbb{V}^{n}$. More precisely, since any erasable $\mathbb{D}$-module is a filtering union of its submodules of finite type, property (1) implies that $\mathscr{L}$ is determined by its restriction to the subcategory of modules of finite type. Any finite type module $M$ is killed by $\mathbb{V}^{n}$ for some $n \geqslant 1$. Since $\mathbb{D} / \mathbb{D} \mathbb{V}^{n}$ is noetherian, for any $M \in C_{n}$ there exist $r, s$ and an exact sequence:

$$
\left(\mathbb{D} / \mathbb{D} \mathbb{V}^{n}\right)^{s} \longrightarrow\left(\mathbb{D} / \mathbb{D} \mathbb{V}^{n}\right)^{r} \longrightarrow M \longrightarrow 0
$$


and for any morphism $f: M \rightarrow M^{\prime}$ in $C_{n}$ there is a commutative diagram:

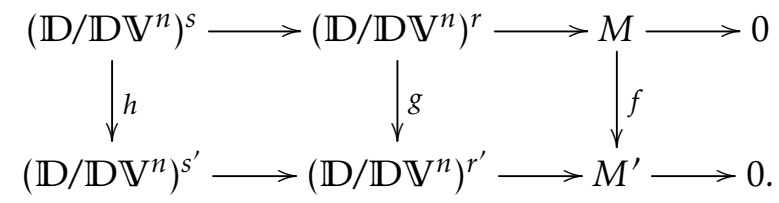

Since $\mathscr{L}$ is right exact, this implies that $\mathscr{L}(M)$ is determined by the values of $\mathscr{L}\left(\left(\mathbb{D} / \mathbb{D} \mathbb{V}^{n}\right)^{r}\right)$ for variable $r$, and $\mathscr{L}(f)$ is determined by the values of $\mathscr{L}(g)$ for variable $g$ as above. Again since $\mathscr{L}$ is right exact, it is additive. Hence $\mathscr{L}\left(\left(\mathbb{D} / \mathbb{D} \mathbb{V}^{n}\right)^{r}\right)$ is determined by $\mathscr{L}\left(\mathbb{D} / \mathbb{D V}^{n}\right)$ which is prescribed in (2). Similarly $\mathscr{L}(g)$ is determined by the values of $\mathscr{L}$ on the various maps $\mathbb{D} / \mathbb{D} \mathbb{V}^{n} \hookrightarrow\left(\mathbb{D} / \mathbb{D} \mathbb{V}^{n}\right)^{r} \rightarrow \mathbb{D} / \mathbb{D} \mathbb{V}^{n}$. Since $\operatorname{End}\left(\mathbb{D} / \mathbb{D} \mathbb{V}^{n}\right)=\mathbb{D} / \mathbb{D} \mathbb{V}^{n}$ is generated by $W(k), F$ and $V$, the assignment in (3) ensures uniqueness of $\mathscr{L}$.

Existence. Because of Proposition 6.2.2, it is enough to check that the functors $\mathscr{L}(M(-))$ and $M(\operatorname{Lie}(-))$ take the same values on the Witt groups $U=W_{n}$ and the endomorphisms of these groups. This is a simple computation which is left to the reader.

We come to the notion of smoothness. It is known that a $k$-group scheme of finite type $U$ is smooth if and only if its relative Frobenius $\mathrm{F}_{U / k}: U \rightarrow U^{(p)}$ is an epimorphism of $k$-group schemes. This motivates the following definition.

6.2.4. Definition. An erasable Dieudonné module $M$ is called smooth if it is of finite type and its Frobenius morphism $\mathrm{F}_{M}: M^{(p)} \rightarrow M$ is a monomorphism.

6.2.5. Definition. An I-extension in $\mathbb{D}$ - $\mathrm{Mod}^{e}$ is an extension of smooth erasable Dieudonné modules of the form

$$
0 \longrightarrow M \longrightarrow M^{\prime} \longrightarrow \operatorname{Lie}(M, I) \longrightarrow 0 \text {. }
$$

A morphism of I-extensions is a morphism of extensions in the usual sense, that is, a morphism of short exact sequences of $\mathbb{D}$-modules.

6.2.6. Theorem. Let $\mathrm{SCU} / k[I]$ be the category of smooth, commutative, unipotent (i.e. with unipotent special fibre) $k[I]$-group schemes. Let $\mathbb{D}-I-M o d$ be the category of I-extensions of smooth erasable Dieudonné modules. Then the Dieudonné functor $M$ induces a contravariant equivalence of categories:

$$
\mathscr{M}: \mathrm{SCU} / k[I] \longrightarrow \mathbb{D}-I-\operatorname{Mod}
$$

that sends $\mathscr{U}$ to the extension $\left.0 \rightarrow \underline{M}\left(\mathscr{U}_{k}\right) \rightarrow \underline{M}\left(h_{*} \mathscr{U}\right)\right) \rightarrow \underline{M}\left(\operatorname{Lie}\left(\mathscr{U}_{k}, I\right)\right) \rightarrow 0$. A quasi-inverse functor is obtained by sending an extension $0 \rightarrow M \rightarrow M^{\prime} \rightarrow \operatorname{Lie}(M, I) \rightarrow 0$ to the Weil extension $h^{+}\left(\underline{U}\left(M^{\prime}\right)\right)$ of the extension $0 \rightarrow \operatorname{Lie}(\underline{U}(M), I) \rightarrow \underline{U}\left(M^{\prime}\right) \rightarrow \underline{U}(M) \rightarrow 0$, where $\underline{U}$ is a quasi-inverse for $\underline{M}$.

Proof. It suffices to put together Theorem A and Theorem 6.1.1. In little more detail, let $\mathscr{U}$ be a smooth, commutative, unipotent group scheme over the ring of dual numbers $k[I]$, and let $U=\mathscr{U}_{k}$ be its special fibre. By Theorem A this datum is equivalent to an extension

$$
0 \longrightarrow \operatorname{Lie}(U, I) \longrightarrow E \longrightarrow U \longrightarrow 0
$$

with $E=h_{*} \mathscr{U}$ smooth, commutative, unipotent. By Theorem 6.1.1 this is equivalent to an extension

$$
0 \longrightarrow \underline{M}(U) \longrightarrow \underline{M}(E) \longrightarrow \underline{M}(\operatorname{Lie}(U, I)) \longrightarrow 0 .
$$

Since $\operatorname{Lie}(\underline{M}(U), I) \stackrel{\sim}{\rightarrow} \underline{M}(\operatorname{Lie}(U, I))$ by Proposition 6.2 .2 , we obtain an $I$-extension as desired. 


\section{Appendix A. Differential calculus on group schemes}

In this appendix we review the notions of tangent bundle and Lie algebra in the required generality. We introduce the exponential morphism of a $k$-group scheme and we establish its main properties, including some special properties needed in the paper. Finally we show how the use of the group algebra allows to recover easily the deformation theory of smooth affine group schemes. Proofs are often omitted, especially in A.1 and A.2.

\section{A.1. Tangent bundle and Lie algebra}

Let $k$ be a ring and $I$ a free $k$-module of finite rank $r \geqslant 1$ with dual $I^{\vee}=\operatorname{Hom}_{k}(I, k)$. Let $k[I]$ be the algebra of dual numbers, i.e. $k[I]:=k \oplus I$ with multiplication determined by the condition $I^{2}=0$. Let $h: \operatorname{Spec}(k[I]) \rightarrow \operatorname{Spec}(k)$ be the structure map and $i: \operatorname{Spec}(k) \rightarrow \operatorname{Spec}(k[I])$ the closed immersion. Basic structure facts on the ring schemes $\mathbb{O}_{k}$ and $\mathbb{O}_{k[I]}$ are recalled in Paragraph 2.1.2.

Let $G$ be a $k$-group scheme with unit section $e: \operatorname{Spec}(k) \rightarrow G$. The tangent bundle of $G / k$ relative to I is the $k$-group scheme defined by:

$$
\mathrm{T}(G, I):=h_{*} h^{*} G .
$$

The $\left(h^{*}, h_{*}\right)$ adjunction (see Subsection 1.3) provides two morphisms of group schemes:

$$
\alpha_{G}: G \longrightarrow \mathrm{T}(G, I) \quad, \quad \beta_{h^{*} G}: h^{*} \mathrm{~T}(G, I)=h^{*} h_{*} h^{*} G \longrightarrow h^{*} G .
$$

From these we derive $\pi_{G}:=i^{*} \beta_{h^{*} G}: \mathrm{T}(G, I) \rightarrow G$ and the Lie algebra of $G / k$ relative to I:

$$
\operatorname{Lie}(G, I):=\operatorname{ker}\left(\pi_{G}\right) .
$$

The map $\mathrm{T}(G, I) \rightarrow G$ is a vector bundle which can be described in terms of derivations. If we identify the $k$-module $I$ with the corresponding locally free sheaf on $\operatorname{Spec}(k)$, then for all points $f: S \rightarrow G$ with values in a $k$-scheme $S$ we have ([SGA3.1, Exposé II, Prop. 3.3]):

$$
\operatorname{Hom}_{G}(S, \mathrm{~T}(G, I)) \stackrel{\sim}{\rightarrow} \operatorname{Der}^{f}\left(\mathcal{O}_{G}, f_{*} \mathcal{O}_{S} \otimes_{k} I\right) .
$$

In particular $\operatorname{Lie}(G, I) \rightarrow \operatorname{Spec}(k)$ is an $\mathbb{O}_{k}$-Lie algebra scheme such that for all $S / k$ we have

$$
\operatorname{Hom}_{k}(S, \operatorname{Lie}(G, I)) \stackrel{\sim}{\rightarrow} \operatorname{Der}^{e}\left(\mathcal{O}_{G}, e_{*} \mathcal{O}_{S} \otimes_{k} I\right) \text {. }
$$

It supports the adjoint representation, i.e. the action of $G$ by conjugation inside $\mathrm{T}(G, I)$ :

$$
\operatorname{Ad}: G \rightarrow \operatorname{GL}(\operatorname{Lie}(G, I)) \quad, \quad \operatorname{Ad}(g)(x)=\alpha_{G}(g) x \alpha_{G}(g)^{-1}
$$

for all points $g$ of $G$ and $x$ of $\operatorname{Lie}(G, I)$. Applying the functor Lie, that is differentiating at the unit section of $G$, we obtain the infinitesimal adjoint representation of the Lie algebra:

$$
\text { ad }: \operatorname{Lie} G \rightarrow \operatorname{End}(\operatorname{Lie}(G, I))) \text {. }
$$

When $I=k \varepsilon$, from ad we deduce the bilinear form called bracket $[-,-]:$ Lie $G \times \operatorname{Lie} G \rightarrow$ Lie $G$. That is, we have $[x, y]:=(\operatorname{ad} x)(y)$ for all points $x, y$ of Lie $G$.

The tangent bundle $\mathrm{T}(G, I)$ carries a structure of extension as follows. By the triangle identity of adjunction, $h^{*} \alpha_{G}$ is a section of $\beta_{h^{*} G}$, hence $\alpha_{G}$ is a section of $\pi_{G}$. Letting $\gamma_{G}$ be the inclusion of $\operatorname{Lie}(G, I)$ into $\mathrm{T}(G, I)$, we have a split exact sequence:

$$
1 \longrightarrow \operatorname{Lie}(G, I) \stackrel{\gamma_{G}}{\longrightarrow} \mathrm{T}(G, I) \underset{\alpha_{G}}{\stackrel{\pi_{G}}{\rightleftarrows}} G \longrightarrow 1
$$


This is an exact sequence of functors, hence also an exact sequence of group schemes (i.e. of fppf sheaves). Let $m$ be the multiplication of $\mathrm{T}(G, I)$. The splitting gives rise to an isomorphism of $k$-schemes:

$$
\rho_{G}: \operatorname{Lie}(G, I) \times G \stackrel{\gamma_{G} \times \alpha_{G}}{\longrightarrow} \mathrm{T}(G, I) \times \mathrm{T}(G, I) \stackrel{m}{\longrightarrow} \mathrm{T}(G, I),
$$

that is, any point of $\mathrm{T}(G, I)$ may be written uniquely as a product $\gamma_{G}(x) \cdot \alpha_{G}(g)$ for some points $x \in \operatorname{Lie}(G, I)$ and $g \in G$. We will sometimes write briefly $(x, g)=\gamma_{G}(x) \cdot \alpha_{G}(g)$ to denote this point of $\mathrm{T}(G, I)$. The conjugation action of $G$ on $\operatorname{Lie}(G, I)$ related to the extension structure is given by the adjoint action, thus the group structure of $\mathrm{T}(G, I)$ can be described by:

$$
(x, g) \cdot\left(x^{\prime}, g^{\prime}\right)=\left(x+\operatorname{Ad}(g) x^{\prime}, g g^{\prime}\right) .
$$

The dependence of $\mathrm{T}(G, I)$ and $\operatorname{Lie}(G, I)$ on $I$ can be described further. If $I=k \varepsilon$ so that $k[I]=k[\varepsilon]$ with $\varepsilon^{2}=0$, we write simply $\mathrm{T} G=\mathrm{T}(G, k \varepsilon)$ and $\operatorname{Lie} G=\operatorname{Lie}(G, k \varepsilon)$ and we call them the tangent bundle and the Lie algebra of $G$. Let $u: G \rightarrow \operatorname{Spec}(k)$ be the structure map. For general $I$, the isomorphisms $\operatorname{Der}^{f}\left(\mathcal{O}_{G}, f_{*} \mathcal{O}_{S}\right) \otimes_{k} I \stackrel{\sim}{\longrightarrow} \operatorname{Der}^{f}\left(\mathcal{O}_{G}, f_{*} \mathcal{O}_{S} \otimes_{k} I\right)$ functorial in $S / G$ induce an isomorphism of vector bundles:

$$
\mathrm{T} G \otimes_{\mathbb{O}_{G}} u^{*} \mathbb{V}\left(I^{\vee}\right) \stackrel{\sim}{\longrightarrow} \mathrm{T}(G, I) .
$$

Similarly, the isomorphisms $\operatorname{Der}\left(\mathcal{O}_{G}, f_{*} \mathcal{O}_{S}\right) \otimes_{k} I \stackrel{\sim}{\rightarrow} \operatorname{Der}\left(\mathcal{O}_{G}, f_{*} \mathcal{O}_{S} \otimes_{k} I\right)$ functorial in $S / k$ induce an isomorphism of $\mathbb{O}_{k}$-Lie algebra schemes:

$$
\text { Lie } G \otimes \mathbb{V}\left(I^{\vee}\right) \stackrel{\sim}{\longrightarrow} \operatorname{Lie}(G, I) .
$$

With $G$ acting trivially on $\mathbb{V}\left(I^{\vee}\right)$, this isomorphism is $G$-equivariant.

A.1.1. Idempotence of Lie. An important property for us will be the idempotence of the Lie functor, namely the existence of an isomorphism $d: \operatorname{Lie}(G, I) \rightarrow \operatorname{Lie}(\operatorname{Lie}(G, I))$ as in [SGA3.1, Exposé II, 4.3.2]. To describe it, let $I, J$ be two finite free $k$-modules. Note that $R[I][J]=R \oplus I R \oplus J R \oplus I J R$. If $G=\operatorname{Spec}(A)$ is affine, for a $k$-algebra $R$, the elements of the set $G(R[I][J])$ are the maps $f: A \rightarrow R[I][J]$ written $f(x)=r(x)+s(x)+t(x)+u(x)$ where $r: A \rightarrow R$ is an algebra map, $s: A \rightarrow I R$ and $t: A \rightarrow J R$ are $r$-derivations, and $u: A \rightarrow I J R$ satisfies the identity:

$$
u(x y)=r(x) u(y)+r(y) u(x)+s(x) t(y)+s(y) t(x) .
$$

Thus $u$ is an $r$-derivation if $s=0$ or $t=0$. Consider the two maps $p: R[I][J] \rightarrow R[I], J \mapsto 0$ and $q: R[I][J] \rightarrow R[J], I \mapsto 0$. Unwinding the definition we see that:

$$
(\operatorname{Lie}(\operatorname{Lie}(G, I), J)(R)=\operatorname{ker}(G(R[I][J]) \stackrel{(p, q)}{\longrightarrow} G(R[I]) \times G(R[J])) .
$$

For varying $R$, the maps $R\left[I \otimes_{k} J\right] \rightarrow R[I][J], i \otimes j \mapsto i j$ induce a morphism of Lie algebra schemes $d: \operatorname{Lie}\left(G, I \otimes_{k} J\right) \longrightarrow \operatorname{Lie}(\operatorname{Lie}(G, I), J)$.

A.1.2. Lemma. The morphism $d: \operatorname{Lie}\left(G, I \otimes_{k} J\right) \rightarrow \operatorname{Lie}(\operatorname{Lie}(G, I), J)$ is an isomorphism.

For simplicity of notation, we will write $d$ as an equality: $\operatorname{Lie}\left(G, I \otimes_{k} J\right)=\operatorname{Lie}(\operatorname{Lie}(G, I), J)$. This will not cause any ambiguity. If $I=J=k \varepsilon$ this means simply that $\operatorname{Lie} G=\operatorname{Lie}(\operatorname{Lie} G)$.

\section{A.2. Exponential and infinitesimal translation}

Demazure and Gabriel in [DG70] use an exponential notation which is flexible enough to coincide in some places with the morphism $\exp _{G}$ as we define it below (loc. cit. chap. II, § 4, 3.7) and in other places with the morphism $\gamma_{G}$ (loc. cit. chap. II, $\S 4,4.2$ ). The drawback of flexibility is a little loss of precision. We introduce the exponential in a somehow more formal way, as an actual morphism between functors. 
A.2.1. Definition. The exponential of a $k$-group scheme $G$ is the composition:

$$
\exp _{G, I}: h^{*} \operatorname{Lie}(G, I) \stackrel{h^{*} \gamma_{G}}{\longrightarrow} h^{*} \mathrm{~T} G \stackrel{\beta_{h^{*} G}}{\longrightarrow} h^{*} G .
$$

When $I$ is clear from context, and also when $I=k \varepsilon$, we write $\exp _{G}$ instead of $\exp _{G, I}$. The following proposition collects some elementary properties of the exponential.

A.2.2. Proposition. The exponential $\exp _{G, I}$ of a $k$-group scheme $G$ has the following properties.

(1. Functoriality) For all morphisms of group functors $f: G \rightarrow G^{\prime}$ we have a commutative square:

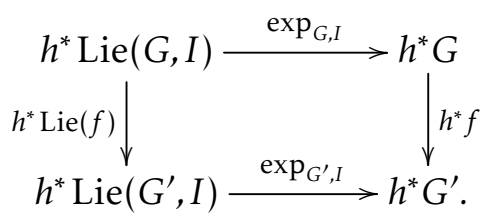

(2. Equivariance) The map $\exp _{G, I}$ is $h^{*} G$-equivariant for the adjoint action on $h^{*} \operatorname{Lie}(G, I)$ and the conjugation action of $h^{*} G$ on itself.

(3. Infinitesimal translation) Let $x$ be a point of $h^{*} \operatorname{Lie}(G, I), g$ a point of $h^{*} G$ and $(x, g)=h^{*} \gamma_{G}(x) \cdot h^{*} \alpha_{G}(g)$ the corresponding point of $h^{*} \mathrm{~T}(G, I)$. Then we have $\beta_{h^{*} G}(x, g)=\exp _{G, I}(x) g$.

(4. Adjoint action of exponentials) Using the description $\operatorname{Lie}(G, I)=$ Lie $G \otimes \mathbb{V}\left(I^{\vee}\right)$, the morphism

$$
h^{*} \operatorname{Ad} \circ \exp _{G, I}: h^{*} \operatorname{Lie}(G, I) \rightarrow h^{*} \mathrm{GL}(\operatorname{Lie}(G, I))
$$

is equal to $x \otimes i \mapsto \mathrm{id}+i \operatorname{ad}(x)$, that is, $\operatorname{Ad}\left(\exp _{G, I}(x \otimes i)\right) x^{\prime}=x^{\prime}+i\left[x, x^{\prime}\right]$.

(5. Exponential of a Lie algebra) Let J be another finite free $k$-module. Using the isomorphism

$$
\operatorname{Lie}(G, J) \otimes \mathbb{V}\left(I^{\vee}\right) \simeq \operatorname{Lie}\left(G, J \otimes_{k} I\right) \stackrel{d}{\rightarrow} \operatorname{Lie}(\operatorname{Lie}(G, J), I)
$$

from Lemma A.1.2, the morphism of $k[I]$-group schemes

$$
h^{*} \operatorname{Lie}(G, J) \otimes_{\mathbb{O}_{k[I]}} h^{*} \mathbb{V}\left(I^{\vee}\right)=h^{*} \operatorname{Lie}(\operatorname{Lie}(G, J), I) \stackrel{\exp _{\operatorname{Lie}(G, J), I}}{\longrightarrow} h^{*} \operatorname{Lie}(G, J)
$$

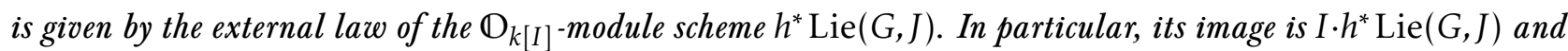
its kernel contains $I \cdot h^{*} \operatorname{Lie}(\operatorname{Lie}(G, J), I)$. Besides, if $I=k \varepsilon$ the map $\exp _{\operatorname{Lie}(G, J)}: h^{*} \operatorname{Lie}(G, J) \rightarrow h^{*} \operatorname{Lie}(G, J)$ is multiplication-by-E in the module scheme $h^{*} \operatorname{Lie}(G, J)$.

(6. Kernel) The two maps:

$$
\exp _{G, I}: h^{*} \operatorname{Lie}(G, I) \longrightarrow h^{*} G, \quad \exp _{\operatorname{Lie}(G), I}: h^{*} \operatorname{Lie}(G, I) \stackrel{h^{*} d}{\simeq} h^{*} \operatorname{Lie}(\operatorname{Lie}(G), I) \longrightarrow h^{*} \operatorname{Lie}(G)
$$

have equal kernels, thus $I \cdot h^{*} \operatorname{Lie}(G, I) \subset \operatorname{ker}\left(\exp _{G, I}\right)$. In particular, in case $I=k \varepsilon$, the kernel of the morphism $\exp _{G}: h^{*}$ Lie $G \rightarrow h^{*} G$ is equal to the kernel of the multiplication-by- $\varepsilon$ map in $h^{*}$ Lie $G$.

We finish this subsection with a corollary of the computation of the exponential of a Lie algebra. 
A.2.3. Lemma. Let $G, H$ be two group schemes over $k$. Let $\phi: G \rightarrow \operatorname{Lie}(H, I)$ be a morphism of pointed schemes. Let $i$ be a section of the ideal $I \cdot \mathbb{O}_{k[I]} \subset \mathbb{O}_{k[I]}$. Then the following compositions are both equal to the trivial morphism:

(1) $h^{*} \operatorname{Lie}(G, I) \stackrel{\exp _{G, I}}{\longrightarrow} h^{*} G \stackrel{h^{*} \phi}{\longrightarrow} h^{*} \operatorname{Lie}(H, I) \stackrel{i}{\longrightarrow} h^{*} \operatorname{Lie}(H, I)$.

(2) $h^{*} \operatorname{Lie}(G, I) \stackrel{\exp _{G, I}}{\longrightarrow} h^{*} G \stackrel{h^{*} \phi}{\longrightarrow} h^{*} \operatorname{Lie}(H, I) \stackrel{\exp _{H, I}}{\longrightarrow} h^{*} H$.

Proof. By functoriality of exp we have a commutative diagram:

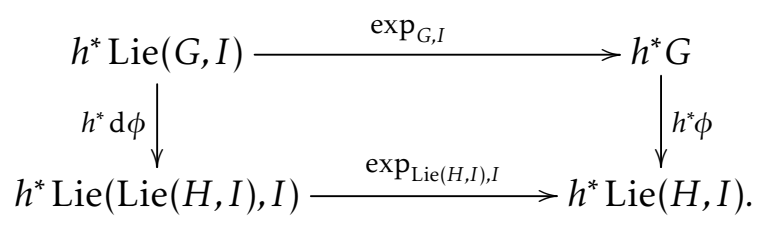

According to Proposition A.2.2(5), the image of $\exp _{\operatorname{Lie}(H, I), I}$ is equal to the subfunctor $I \cdot h^{*} \operatorname{Lie}(G, I)$. Since $I^{2}=0$, from this (1) follows. Moreover, by A.2.2(6) the map $\exp _{H, I}$ has the same kernel as $\exp _{\operatorname{Lie}(H), I}$ which by A.2.2(5) contains $I \cdot h^{*} \operatorname{Lie}(H, I)$. Again, since $I^{2}=0$, point (2) follows.

\section{A.3. Deformations of affine group schemes}

In this subsection, we illustrate the usefulness of the group algebra in two ways. First, in Proposition A.3.1 we show how concepts of differential calculus can be handled very conveniently using the group algebra. We include the examples of the adjoint action and the Lie bracket. The results of Proposition A.2.2 can be derived painlessly in a similar fashion. Then in Proposition A.3.3 we show how to recover directly the fact that isomorphism classes of deformations over $k[I]$ of a smooth, affine $k$-group scheme $G$ are classified by the second cohomology group $\mathrm{H}^{2}(G, \operatorname{Lie}(G, I))$.

A.3.1. Proposition. Let $G$ be an affine $k$-group scheme and $\left(\mathbb{O}_{k}[G],+, \star\right)$ its group algebra.

(1) The tangent bundle $\mathrm{T}\left(\mathbb{O}_{k}[G], I\right)$ of the group algebra $\mathbb{O}_{k}[G]$ is canonically isomorphic to the algebra scheme $\mathbb{O}_{k}[G][I]=\mathbb{O}_{k}[G] \oplus \mathbb{O}_{k}[G] \cdot I$. We have a commutative diagram of affine monoid schemes:

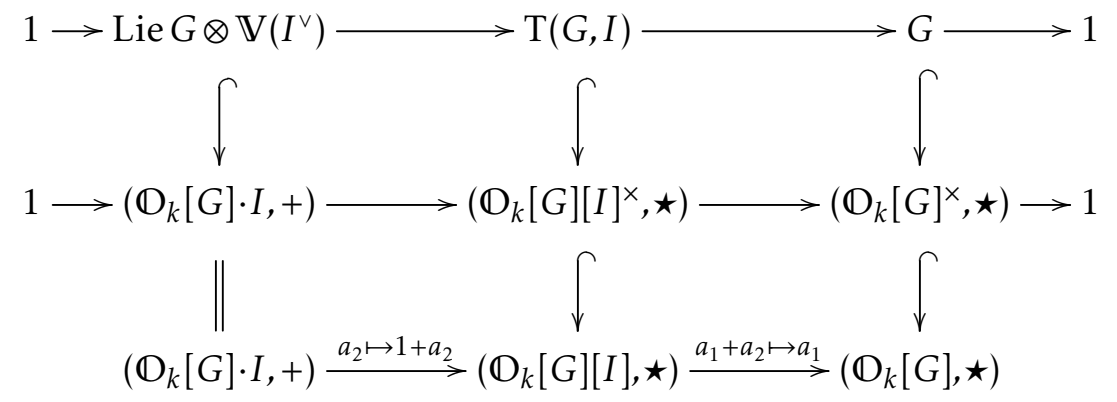

The first two rows are split exact sequences of group schemes. In the last row we have written the points of $\mathbb{O}_{k}[G][I]$ as $a=a_{1}+a_{2}$ with $a_{1} \in \mathbb{O}_{k}[G]$ and $a_{2} \in \mathbb{O}_{k}[G] \cdot I$. The map Lie $G \otimes \mathbb{V}\left(I^{\vee}\right) \hookrightarrow\left(\mathbb{O}_{k}[G] \cdot I,+\right)$ induces an isomorphism between Lie $G \otimes \mathbb{V}\left(I^{\vee}\right)$ and the subscheme $\operatorname{Der}_{G}^{1} \subset \mathbb{O}_{k}[G]$ of e-derivations where e is the neutral element of $G$.

(2) The adjoint action $\mathrm{Ad}: G \rightarrow \mathrm{GL}(\operatorname{Lie}(G, I))$ can be expressed as a conjugation inside $\mathbb{O}_{k}[G][I]$ :

$$
1+\operatorname{Ad}(g) x=g(1+x) g^{-1}=1+g x g^{-1},
$$

for all $x \in \operatorname{Lie}(G, I)=\operatorname{Lie} G \otimes \mathbb{V}\left(I^{\vee}\right)$ and $g \in G$.

(3) The Lie bracket $[-,-]:$ Lie $G \times \operatorname{Lie} G \rightarrow$ Lie $G$ can be expressed as the bracket defined by the associative multiplication of $\mathrm{O}_{k}[G]$ :

$$
[x, y]=x y-y x
$$


A.3.2. Remark. More generally, for all finite free $k$-modules $I, J$ we have an infinitesimal adjoint action

$$
\text { ad }:=\operatorname{Lie}(\operatorname{Ad}, J): \operatorname{Lie}(G, J) \rightarrow \operatorname{Hom}(\operatorname{Lie}(G, I), \operatorname{Lie}(G, I \otimes J))
$$

which can be expressed as the bracket defined by the associative multiplication of $\mathbb{O}_{k}[G][I][J]$, that is, $[x, y]=x y-y x$ for $x \in \mathbb{O}_{k}[G] \cdot J$ and $y \in \mathbb{O}_{k}[G] \cdot I$. The expression in the particular case $I=J=k \varepsilon$ is obtained by writing $1+\varepsilon x$ instead of $1+x$.

Proof. (1) By Proposition 2.3.1(4), we have an isomorphism of $\mathbb{O}_{k}$-algebra schemes:

$$
\mathrm{T}\left(\mathbb{O}_{k}[G], I\right)=h_{*} h^{*} \mathbb{O}_{k}[G] \stackrel{\sim}{\rightarrow} \mathbb{O}_{k}[G] \otimes_{\mathbb{O}_{k}} h_{*} \mathbb{O}_{k[I]} \simeq \mathbb{O}_{k}[G][I] .
$$

Under this identification, the map $\mathrm{T} v_{G}: \mathrm{T}(G, I) \rightarrow \mathrm{T}\left(\mathbb{O}_{k}[G], I\right)=\mathbb{O}_{k}[G][I]$ can be described as follows: for each $k$-algebra $R$, a point $f \in \mathrm{T}(G, I)(R)$ is a morphism $f: A \rightarrow R[I], f(x)=u(x)+v(x)$ for some unique $k$-module homomorphisms $u: A \rightarrow R$ and $v: A \rightarrow I R$, and we have

$$
\mathrm{T} v_{G}(f)=u+v \in \mathbb{O}_{k}[G][I](R) .
$$

This is the central vertical map in the pictured diagram. The rest is clear.

(2) Using the inclusions of multiplicative monoids $\alpha_{G}: G \hookrightarrow \mathrm{T} G$ and $\mathrm{T} \nu_{G}: \mathrm{T} G \hookrightarrow \mathbb{O}_{k}[G][\varepsilon]$, we can view the conjugation action by $G$ inside the tangent bundle or inside the tangent group algebra, as we wish. The result follows.

(3) In order to compute ad we differentiate and hence work in $\mathbb{O}_{k}[G]\left[\varepsilon, \varepsilon^{\prime}\right]$. That is, the Lie algebra embedded by $y \mapsto 1+\varepsilon y$ is acted upon by the Lie algebra embedded by $x \mapsto 1+\varepsilon^{\prime} x$, via conjugation in the ambient $\mathbb{O}_{k}[G]\left[\varepsilon, \varepsilon^{\prime}\right]$. With these notations, the identification $\operatorname{End}(\operatorname{Lie} G) \stackrel{\sim}{\longrightarrow} \operatorname{Lie}(G L(\operatorname{Lie} G))$ goes by $f \mapsto 1+\varepsilon^{\prime} f$. All in all, the outcome is that ad $(x)$ is determined by the condition that for all $y$ we have:

$$
\left(1+\varepsilon^{\prime} x\right)(1+\varepsilon y)\left(1-\varepsilon^{\prime} x\right)=1+\varepsilon\left(\left(\mathrm{id}+\varepsilon^{\prime} \operatorname{ad}(x)\right)(y)\right) .
$$

Since the left-hand side is equal to $1+\varepsilon y+\varepsilon \varepsilon^{\prime}(x y-y x)$, this proves our claim.

A.3.3. Proposition. Let $k$ be a base ring and let $G$ be an affine $k$-group scheme.

(1) The set of $k[I]$-group scheme structures on the scheme $h^{*} G$ that lift the $k$-group scheme structure of $G$ is in bijection with the set of 2-cocycles $c: G \times G \rightarrow$ Lie $G$.

(2) The set of isomorphism classes of rigid deformations of $G$ over $k[I]$ is in bijection with $\mathrm{H}^{2}(G, \mathrm{Lie}(G, I))$, the second group cohomology of $G$ with coefficients in the adjoint representation $\operatorname{Lie}(G, I) \simeq \operatorname{Lie} G \otimes \mathbb{V}\left(I^{\vee}\right)$.

Proof. (1) We want to deform the multiplication $m: G \times G \longrightarrow G, \quad(u, v) \mapsto u v$ into a multiplication $\widetilde{m}: h^{*} G \times h^{*} G \rightarrow h^{*} G$ which by adjunction we can view as a map:

$$
G \times G \rightarrow h_{*} h^{*} G=\mathrm{T}(G, I), \quad(u, v) \mapsto u \odot v .
$$

We use embeddings inside the group algebra as in Proposition A.3.1; thus both targets of $m$ and $\widetilde{m}$ are embedded in $\mathbb{O}_{k}[G][I]$. The condition that $\widetilde{m}$ equals $m$ modulo $I$ is that these morphisms agree after composition with the projection $\pi: \mathbb{O}_{k}[G][I] \rightarrow \mathbb{O}_{k}[G]$. In other words the condition is that for all points $u, v \in G$, the element $(u \odot v)(u v)^{-1}$ equals 1 modulo $I$. Since this is also a point of $\mathrm{T}(G, I)$, it belongs to $\operatorname{Lie}(G, I)$. Hence we can write

$$
u \odot v=(1+c(u, v)) u v
$$

for some $c: G \times G \rightarrow \operatorname{Lie}(G, I)$. The associativity constraint $(u \odot v) \odot w=u \odot(v \odot w)$ gives the cocycle relation:

$$
c(u v, w)+c(u, v)=c(u, v w)+u c(v, w) u^{-1}
$$


Conversely, if $c: G \times G \rightarrow \operatorname{Lie}(G, I)$ is a 2-cocycle, we define

$$
u \odot v:=(1+c(u, v)) u v .
$$

The cocycle identity gives the associativity of this map. Moreover, inverses for this law exist and are given by the formula $u^{\odot-1}=u^{-1}\left(1-c\left(u, u^{-1}\right)\right)$. So we obtain a $k[I]$-group scheme $\mathscr{G}_{c}:=(\operatorname{Spec}(A[I]), \odot)$ which is a deformation of $G$.

(2) Let $\mathscr{G}$ be a rigid deformation of $G$. Choosing an isomorphism of schemes $\varphi_{1}: \mathscr{G} \stackrel{\sim}{\rightarrow} h^{*} G$, the induced group scheme structure on $h^{*} G$ gives rise to a cocycle $c$ as explained in (1). Choosing another isomorphism $\varphi_{2}: \mathscr{G} \stackrel{\sim}{\rightarrow} h^{*} G$, we have an automorphism $\xi=\varphi_{2} \circ \varphi_{1}^{-1}: h^{*} G \rightarrow h^{*} G$ which restricts to the identity on $G$. The map $G \rightarrow h_{*} h^{*}=\mathrm{T}(G, I)$ obtained by adjunction is of the form $u \mapsto(1+\psi(u)) u$ for some morphism $\psi: G \rightarrow \operatorname{Lie}(G, I)$. This means that $\xi(u)=(1+\psi(u)) u$. We want to see how the multiplication is transformed by the change of isomorphism. If $u^{\prime}=(1+\psi(u)) u$ then $u=\left(1-\psi\left(u^{\prime}\right)\right) u^{\prime}$, a short computation shows that that the map $\xi \circ \odot \circ \xi^{-1}$ takes $\left(u^{\prime}, v^{\prime}\right)$ to $\left[1+\left(\psi\left(u^{\prime} v^{\prime}\right)+c\left(u^{\prime}, v^{\prime}\right)-\psi\left(u^{\prime}\right)-\operatorname{Ad}\left(u^{\prime}\right) \psi\left(v^{\prime}\right)\right)\right] u^{\prime} v^{\prime}$. We see that $c$ changes by the coboundary $\partial \psi$ and the class $[c] \in \mathrm{H}^{2}(G, \operatorname{Lie}(G, I))$ does not depend on the choice of isomorphism $\mathscr{G} \stackrel{\sim}{\rightarrow} h^{*} G$. To obtain the inverse bijection, one chooses a cocycle $c$ and attaches the deformation $\mathscr{G}_{c}$ as in (1). The map is well-defined because another choice of $c$ in the same cohomology class gives an isomorphic deformation.

\section{Appendix B. Module stacks in groupoids}

Both categories $\mathrm{Gr} / k[I]$ and $\operatorname{Ext}(I) / k$ are endowed with the structure of $\mathbb{O}_{k}$-module stacks in groupoids over $\mathrm{Gr} / k$ and the purpose of this Appendix is to explain what this means. In the two cases this seems to be a well-known fact, but we were able to locate only very few discussions of this topic in the literature. In fact, the additive part of the structure, which goes by the name of a "Picard category", is well documented, a landmark being Deligne's exposé in [SGA4.3], Exp. XVIII, § 1.4. However, the linear part of the structure, that is the $\mathbb{O}_{k}$-scalar multiplication and its interplay with the additive structure, is almost absent from the literature. Subsections 2.3, 2.4 and 2.5 of Osserman [Os10] are a first step, but the author writes: Although it is possible to [define scalar multiplication maps] on a categorical level as we did with addition, expressing the proper conditions for associativity and distributivity isomorphisms becomes substantially more complicated. Here we simply provide a definition in due form. An extended version of the article available on the authors' webpage contains a treatment including a few basic results to highlight the nontrivial features of the theory.

We start with the definition of Picard categories. The alternative phrase commutative group groupoids is a more accurate name to refer to them, but we stick with the traditional name. In order to make the axioms reader-friendly, we adopt a simplified description for the multifunctors involved, e.g. the associativity isomorphism $a: T_{1} \rightarrow T_{2}$ between the trifunctors $T_{1}, T_{2}: P \times P \times P \rightarrow P$ given by $T_{1}=+\circ(+\times \mathrm{id})$ and $T_{2}=+\circ(\mathrm{id} \times+)$ is given in the form of isomorphisms $a_{x, y, z}:(x+y)+z \rightarrow x+(y+z)$ functorial in $x, y, z \in P$.

B.1. Definition. Let $P$ be a category and $+: P \times P \rightarrow P$ a bifunctor.

(1) An associativity constraint for + is an isomorphism of functors $a_{x, y, z}:(x+y)+z \stackrel{\sim}{\rightarrow} x+(y+z)$ such that the pentagon axiom ([SGA4.3], Exp. XVIII, 1.4.1) is satisfied. It is called trivial or strict if $a_{x, y, z}=$ id for all $x, y, z \in P$.

(2) A commutativity constraint for + is an isomorphism of functors $c_{x, y}: x+y \stackrel{\sim}{\rightarrow} y+x$ which satisfies $c_{y, x} \circ c_{x, y}=\operatorname{id}_{x+y}$ for all $x, y \in P$. It is called trivial or strict if $c_{x, y}=$ id for all $x, y \in P$.

(3) The associativity and commutativity constraints $a$ and $c$ are compatible if the hexagon axiom ([SGA4.3], Exp. XVIII, 1.4.1) is satisfied.

(4) A neutral element for + is an object $0 \in P$ with an isomorphism $\varphi: 0+0 \stackrel{\sim}{\longrightarrow} 0$.

In other mathematical contexts, associativity constraints are called associators and commutativity constraints are called symmetric braidings. 
B.2. Definition. Let $\left(P_{1},+\right)$ and $\left(P_{2},+\right)$ be categories endowed with bifunctors. Let $F: P_{1} \rightarrow P_{2}$ be a functor and $\varphi_{F, x, y}: F(x+y) \stackrel{\sim}{\rightarrow} F(x)+F(y)$ an isomorphism of functors.

(1) Let $a_{1}, a_{2}$ be associativity constraints on $\left(P_{1},+\right)$ and $\left(P_{2},+\right)$. We say that $\left(F, \varphi_{F}\right)$ is compatible with $a_{1}, a_{2}$ if the following diagram commutes:

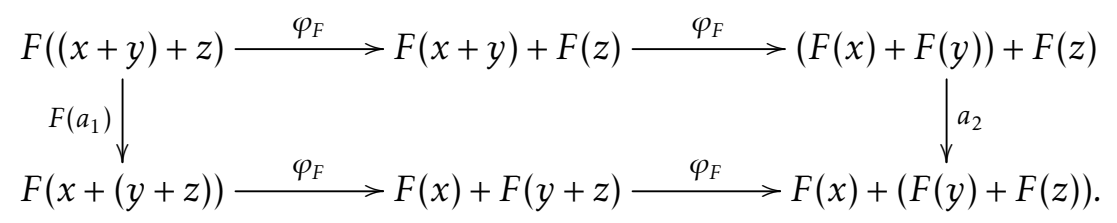

(2) Let $c_{1}, c_{2}$ be commutativity constraints on $\left(P_{1},+\right)$ and $\left(P_{2},+\right)$. We say that $\left(F, \varphi_{F}\right)$ is compatible with $c_{1}, c_{2}$ if the following diagram commutes:

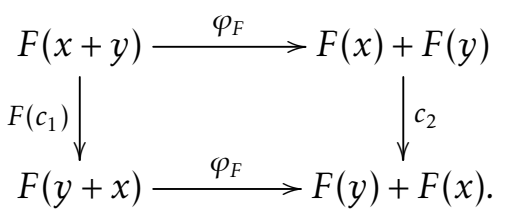

B.3. Definition. A Picard category is a quadruple $(P,+, a, c)$ composed of a nonempty groupoid $P$, a bifunctor $+: P \times P \rightarrow P$ with compatible associativity and commutativity constraints $a$ and $c$, such that for each $x \in P$ the functor $P \rightarrow P, y \mapsto x+y$ is an equivalence.

Any Picard category $P$ has a neutral element 0 which is unique up to a unique isomorphism ([SGA4.3], Exp. XVIII, 1.4.4). Moreover, for each $x, y \in P$ the set of morphisms $\operatorname{Hom}(x, y)$ is either empty or a torsor under the group $G:=\operatorname{Aut}(0)$. More precisely, the functors $+x: P \rightarrow P$ and $x+: P \rightarrow P$ induce the same bijection $G \rightarrow \operatorname{Aut}(x), \varphi \mapsto \varphi+\mathrm{id}_{x}$. Viewing this bijection as an identification, the set $\operatorname{Hom}(x, y)$ with its right $\operatorname{Aut}(x)$-action and left $\operatorname{Aut}(y)$-action becomes a pseudo-G-bitorsor, i.e. it is either empty or a G-bitorsor.

B.4. Definition. Let $P_{1}, P_{2}$ be Picard categories.

(1) An additive functor is a pair $\left(F, \varphi_{F}\right)$ where $F: P_{1} \rightarrow P_{2}$ is a functor and $\varphi_{F, x, y}: F(x+y) \stackrel{\sim}{\rightarrow} F(x)+F(y)$ is an isomorphism of functors that is compatible with associativity and commutativity constraints.

(2) Let $F, G: P_{1} \rightarrow P_{2}$ be additive functors. A morphism of additive functors is a morphism of functors $u: F \rightarrow G$ such that the following diagram is commutative:

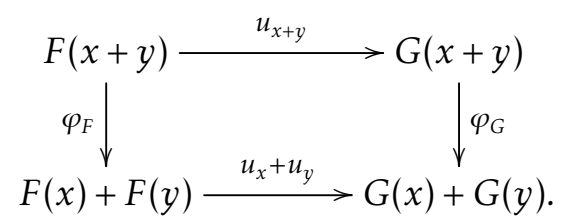

We emphasize that since a Picard category is a groupoid (that is, all its morphisms are isomorphisms), all morphisms of additive functors $u: F \rightarrow G$ are isomorphisms.

The category of additive functors $\operatorname{Hom}\left(P_{1}, P_{2}\right)$ is itself a Picard category ([SGA4.3], Exp. XVIII, 1.4.7). Additive functors can be composed and the identity functors behave as neutral elements. In the particular case where $P_{1}=P_{2}=P$, along with its addition law, the Picard category $\operatorname{End}(P)=\operatorname{Hom}(P, P)$ enjoys an internal multiplication given by composition. Note that in this case multiplication is strictly associative, because so is composition of functors in categories.

In fact $\operatorname{End}(P)$ is a ring category, but in order to introduce module groupoids, we do not actually need to define what is such a thing. 
B.5. Definition. Let $\Lambda$ be a commutative ring. A $\Lambda$-module groupoid is a Picard category $P$ endowed with a functor $F=\left(F, \varphi_{F}, \psi_{F}\right): \Lambda \rightarrow \operatorname{End}(P)$ called scalar multiplication such that:

(1) $\left(F, \varphi_{F}\right)$ is an additive functor.

For each $\lambda \in \Lambda$, for simplicity we write $\left(\lambda, \varphi_{\lambda}\right)$ for $\left(F_{\lambda}, \varphi_{F_{\lambda}}\right): P \rightarrow P$. Moreover:

(2) $\left(F, \psi_{F}\right)$ is multiplicative, i.e. $F(1)=\operatorname{id}_{P}$ and $F$ is compatible with the associativity constraints of multiplication.

(3) $F$ is compatible with the distributivity of multiplication over addition:

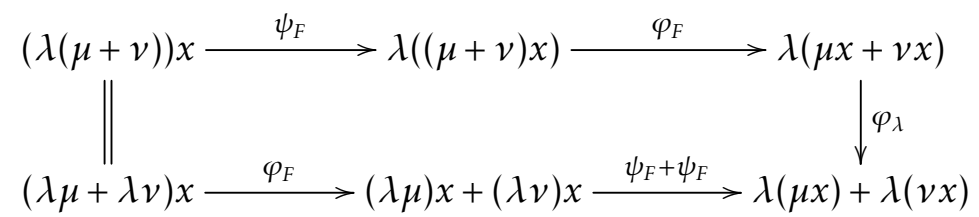

commutes.

B.6. Definition. Let $\mathcal{S}$ be a site. Let $\Lambda$ be a sheaf of commutative rings on $\mathcal{S}$. A $\Lambda$-module stack (in groupoids) over $\mathcal{S}$ is a stack in groupoids $P$ over $\mathcal{S}$ endowed with

(1) a functor $+: P \times P \rightarrow P$,

(2) isomorphisms of functors $a_{x, y, z}:(x+y)+z \stackrel{\sim}{\rightarrow} x+(y+z)$ and $c_{x, y}: x+y \stackrel{\sim}{\rightarrow} y+x$,

(3) a functor $F=\left(F, \varphi_{F}, \psi_{F}\right): \Lambda \rightarrow \operatorname{End}(P)$,

such that for each $U \in \mathcal{S}$ the fibre category $P(U)$ is a $\Lambda(U)$-module groupoid.

There is an obvious corresponding relative notion of $\Lambda$-module stack (in groupoids) over a given $\mathcal{S}$-stack $Q$, namely, it is a morphism of stacks $P \rightarrow Q$ that makes $P$ a stack fibred in groupoids over $Q$, with an addition functor $+: P \times_{Q} P \rightarrow P$ etc. It is the relative notion that is useful in the paper.

\section{References}

[BLR90] S. Bosch, W. Lütkebohmert, and M. Raynaud, Néron models, Ergebnisse der Mathematik und ihrer Grenzgebiete (3), vol. 21, Springer-Verlag, Berlin, 1990. MR-1045822

[DG70] M. Demazure and P. Gabriel, Groupes algébriques, Tome I: Géométrie algébrique, généralités, groupes commutatifs, Masson, Paris; North-Holland Publishing, Amsterdam, 1970. MR-0302656

$\left[E G A 1_{\text {new }}\right]$ A. Grothendieck and J. Dieudonné, Eléments de géométrie algébrique I, Grundlehren der Mathematischen Wissenschaften, vol. 166, Springer-Verlag, Berlin, 1971. MR-3075000

[Fo77] J.-M. Fontaine, Groupes p-divisibles sur les corps locaux, Astérisque, No. 47-48, Société Mathématique de France, Paris, 1977. MR-0498610

[I172] L. Illusie, Complexe cotangent et déformations II, Lecture Notes in Mathematics, vol. 283, SpringerVerlag, Berlin-New York, 1972. MR-0491681

[1185] L. Illusie, Déformations de groupes de Barsotti-Tate (d'après A. Grothendieck). In: Seminaire sur les pinceaux arithmétiques: la conjecture de Mordell (Paris, 1983/84), pp. 151-198, Astérisque No. 127, Société Mathématique de France, Paris, 1985. MR-0801922

[MRT13] A. Mézard, M. Romagny, and D. Tossici, Models of group schemes of roots of unity, Ann. Inst. Fourier (Grenoble) 63 (2013), no. 3, 1055-1135. MR-3137480 
[Os10] B. Osserman, Deformations and automorphisms: a framework for globalizing local tangent and obstruction spaces, Ann. Sc. Norm. Super. Pisa Cl. Sci. (5) 9 (2010), no. 3, 581-633. MR-2722657

[Per76] D. Perrin, Approximation des schémas en groupes, quasi compacts sur un corps, Bull. Soc. Math. France 104 (1976), no. 3, 323-335. MR-0432661

[SGA3.1] Séminaire de Géométrie Algébrique du Bois Marie 1962-64 (SGA3). Schémas en groupes. Tome I. Propriétés générales des schémas en groupes. A seminar directed by M. Demazure and A. Grothendieck with the collaboration of M. Artin, J.-E. Bertin, P. Gabriel, M. Raynaud, and J-P. Serre. Revised and annotated edition of the 1970 French original. Edited by Philippe Gille and Patrick Polo. Documents Mathématiques, vol. 7, Société Mathématique de France, Paris, 2011. MR-2867621

[SGA4.3] Séminaire de Géométrie Algébrique du Bois Marie 1963-64 (SGA4). Théorie des topos et cohomologie étale des schémas. Tome 3. Dirigé par M. Artin, A. Grothendieck et J. L. Verdier. Avec la collaboration de P. Deligne et B. Saint-Donat. Lecture Notes in Mathematics, vol. 305, Springer-Verlag, Berlin-New York, 1973. MR-0354654

[SP] The Stacks Project Authors, The stacks project. https://stacks.math.columbia.edu/ 